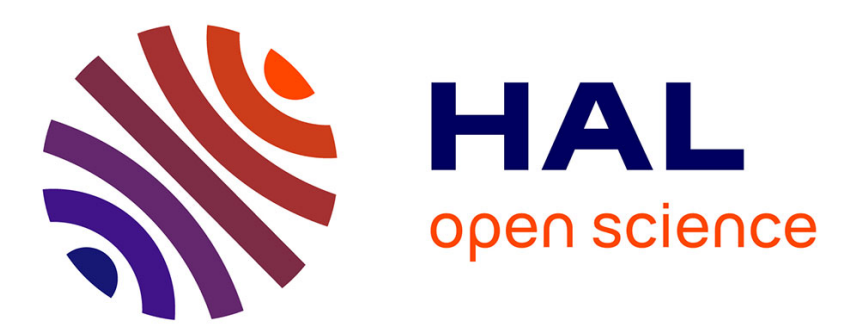

\title{
A Continuation Problem for Computing Solutions of Discretised Evolution Problems with Application to Plane Quasi-Static Contact Problems with Friction
}

\author{
Tomas Ligursky, Yves Renard
}

\section{- To cite this version:}

Tomas Ligursky, Yves Renard. A Continuation Problem for Computing Solutions of Discretised Evolution Problems with Application to Plane Quasi-Static Contact Problems with Friction. Computer Methods in Applied Mechanics and Engineering, 2014, 280, pp.222-262. 10.1016/j.cma.2014.07.011. hal-00877716v2

\author{
HAL Id: hal-00877716 \\ https://hal.science/hal-00877716v2
}

Submitted on 10 Jul 2014

HAL is a multi-disciplinary open access archive for the deposit and dissemination of scientific research documents, whether they are published or not. The documents may come from teaching and research institutions in France or abroad, or from public or private research centers.
L'archive ouverte pluridisciplinaire HAL, est destinée au dépôt et à la diffusion de documents scientifiques de niveau recherche, publiés ou non, émanant des établissements d'enseignement et de recherche français ou étrangers, des laboratoires publics ou privés. 


\title{
A Continuation Problem for Computing Solutions of Discretised Evolution Problems with Application to Plane Quasi-Static Contact Problems with Friction
}

\author{
Tomáš Ligurskýa,* $\quad$ Yves Renard $^{b}$
}

October 23, 2013 (revised version March 21, 2014)

\footnotetext{
${ }^{a}$ Department of Mathematical Analysis and Applications of Mathematics, Faculty of Science, Palacký University, 17. listopadu 12, 77146 Olomouc, Czech Republic. tomas.ligursky@upol.cz

${ }^{b}$ Université de Lyon, CNRS, INSA-Lyon, ICJ UMR5208, F 69621, Villeurbanne, France. Yves.Renard@insa-lyon.fr
}

\begin{abstract}
A continuation problem for finding successive solutions of discretised abstract first-order evolution problems is proposed and a general piecewise $C^{1}$ continuation problem is studied. A condition ensuring local existence and uniqueness of its solution curves is given. An analogy of the first-order system of smooth problems is derived and results of existence and uniqueness of its solutions are stated. Possibility of continuation of a solution curve along directions solving the first-order system is discussed. A technique for numerical continuation of the solution curves is developed. Furthermore, an application of the abstract continuation problem is presented for plane quasi-static contact problems with friction. Various formulations of the firstorder system are derived for this case so that the analysis from the abstract frame can be developed and supplemented. Finally, the proposed numerical continuation is tested.
\end{abstract}

Keywords: continuation, piecewise-smooth system, first-order system, predictor-corrector, contact problem, Coulomb friction.

\section{Introduction}

When time-stepping schemes are used to solve quasi-static problems in solid mechanics numerically, one can encounter situations where usual solvers (for instance, the Newton method with the initial approximation chosen to be a solution from the previous time step)

${ }^{*}$ Corresponding author. Tel.: (+420) 585634606. 
fail to compute any solution. Typically, this can happen when a snap-through instability is present and even a small change in loading leads to a dramatic change of the solution. This has lead us to construct a suitable continuation problem for dealing with such situations. Although our motivation originated from solving quasi-static problems with the particularity that the time derivative appears only in a nonlinear term, our approach can be applied to first-order evolution problems generally. The idea how to do it is explained in an abstract frame in the beginning of Section 2.

Whereas continuation is well-established for problems involving a continuously differentiable map (see [4], for example), a little work has been done for problems with general non-smooth functions and it is oriented mainly to homotopy methods [3, 28]. That is why the next ambition of this paper is to give a rigorous analysis of a general continuation problem. In particular, a problem involving an arbitrary piecewise $C^{1}\left(P C^{1}\right)$ function is considered in Subsection 2.1, and a result guaranteeing local existence and uniqueness of its solution curves is stated. Furthermore, an analogy of the first-order system for smooth problems is introduced, which gives a possibility of studying tangent behaviour of solution curves near a given solution point.

The reason why we confine ourselves to the framework of $P C^{1}$-functions is that it seems to be well-suited for plane contact problems, for which a particular continuation problem is proposed in Section 3. Contact problems lead to functions that are not Gâteauxdifferentiable in general. Nevertheless, let us note that other problems from engineering or economics are covered by the framework, as well [26].

After laying theoretical foundation, we describe a method of numerical continuation for tracing $P C^{1}$ solution curves in Subsection 2.2. Our approach is close to the continuation from [28] for normal maps with polyhedral convex sets, to the ones from [6, 27] for frictionless contact problems or to the ones from $[14,12,11]$ for plane contact problems with Coulomb friction. The main contrast to all those papers is that the present algorithm does not obey precise expressions of sub-domains of smooth behaviour of the $P C^{1}$-function involved, which require quite detailed specification of the function during implementation.

The strategy proposed here is based on the predictor-corrector method for smooth functions sketched in [8], which resembles an arc-length continuation and is capable of traversing smooth folds on its own, without adding any special routines. Having inherited this property, our continuation includes also a special technique for treating non-smooth points on solution curves, which may be even folds additionally. Let us point out that we use no smoothing unlike $[29,15]$ to avoid the danger of modification of the solution structure of the original non-smooth problem.

As indicated, Section 3 deals with a particular continuation problem for discretised quasi-static plane contact problems with Coulomb friction in large deformations. Firstly, we formulate the corresponding continuous problem and discretise it. The spatial discretisation is done by a mixed finite-element method while time derivatives are approximated by backward differences. Then we propose a continuation problem for this case and making use of ideas from [2], we reformulate it so that it fits the general framework.

Subsection 3.1 employs the specific structure of the problem and establishes more precise analysis of its first-order system, extending the studies [6, 27, 19] for frictionless prob- 
lems. In particular, we formulate the first-order system in such a way that it is close to a rate problem of a quasi-static contact problem from the mathematical point of view. Making use of this similarity, we adapt the analysis of the rate problem from [22] to our first-order system. Moreover, we investigate the abstract result of continuation of solution curves in directions solving the first-order system.

Finally, numerical experiments with the continuation method from Subsection 2.2 are presented in Subsection 3.2. A similar algorithm has already been tested on static contact problems in $[14,12]$, but only on finite-element models with very small number of degrees of freedom. Here we show results for more realistic models.

\subsection{Notation and Preliminaries}

The following notation is employed throughout the paper: For a vector $\boldsymbol{x} \in \mathbb{R}^{N}$, a matrix $\boldsymbol{A} \in \mathbb{R}^{M \times N}$ and index sets $I \subset\{1, \ldots, M\}$ and $J \subset\{1, \ldots, N\}, x_{i}$ stands for the $i$ th component of $\boldsymbol{x}, \boldsymbol{x}_{J}$ is the sub-vector of $\boldsymbol{x}$ composed from the components $x_{i}, i \in J$, and $\boldsymbol{A}_{I, J}$ is the sub-matrix of $\boldsymbol{A}$ with rows and columns specified by $I$ and $J$, respectively. Furthermore, $\boldsymbol{x} \cdot \boldsymbol{y}=\boldsymbol{x}^{\top} \boldsymbol{y}$ is a scalar product of vectors $\boldsymbol{x}$ and $\boldsymbol{y},\|\boldsymbol{x}\|$ denotes the Euclidean norm of $\boldsymbol{x}$ and $B(\boldsymbol{x}, r)$ stands for a closed ball centred at $\boldsymbol{x}$ with radius $r$.

The gradients of a real-valued function $f$ and a vector-valued function $\boldsymbol{f}$ at a point $\overline{\boldsymbol{x}}$ are denoted by $\nabla f(\overline{\boldsymbol{x}})$ and $\boldsymbol{\nabla} \boldsymbol{f}(\overline{\boldsymbol{x}})$, respectively, the partial gradients with respect to $\boldsymbol{y}$ of $f$ and $\boldsymbol{f}$ at $(\overline{\boldsymbol{x}}, \overline{\boldsymbol{y}})$ are denoted by $\nabla_{\boldsymbol{y}} f(\overline{\boldsymbol{x}}, \overline{\boldsymbol{y}})$ and $\boldsymbol{\nabla}_{\boldsymbol{y}} \boldsymbol{f}(\overline{\boldsymbol{x}}, \overline{\boldsymbol{y}})$, respectively, and $\nabla^{2} f(\overline{\boldsymbol{x}})$ is the Hessian of $f$ at $\overline{\boldsymbol{x}}$. If $\boldsymbol{f}$ is a function of a real variable, $\boldsymbol{f}^{\prime}$ denotes its right-hand derivative for brevity of notation. In the same way as for vectors, the $j$ th component function of a vector-valued function $\boldsymbol{f}$ is systematically denoted by $f_{j}$.

For reader's convenience, we recall essentials from theory of $P C^{1}$-functions [26, 24]:

Definition 1. (i) A function $\boldsymbol{H}: \mathbb{R}^{M} \rightarrow \mathbb{R}^{N}$ is $P C^{1}$ if it is continuous and for every $\overline{\boldsymbol{y}} \in \mathbb{R}^{M}$, there exist an open neighbourhood $O$ of $\overline{\boldsymbol{y}}$ and a finite family of $C^{1}$-functions $\boldsymbol{H}^{(i)}: O \rightarrow \mathbb{R}^{N}, i \in \mathcal{I}$, such that

$$
\forall \boldsymbol{y} \in O: \quad \boldsymbol{H}(\boldsymbol{y}) \in\left\{\boldsymbol{H}^{(i)}(\boldsymbol{y}) ; i \in \mathcal{I}\right\} .
$$

The functions $\boldsymbol{H}^{(i)}$ are termed selection functions of $\boldsymbol{H}$ at $\overline{\boldsymbol{y}}$.

(ii) A selection function $\boldsymbol{H}^{(i)}$ of a $P C^{1}$-function $\boldsymbol{H}$ is called active at $\overline{\boldsymbol{y}}$ if

$$
\boldsymbol{H}^{(i)}(\overline{\boldsymbol{y}})=\boldsymbol{H}(\overline{\boldsymbol{y}})
$$

and essentially active at $\overline{\boldsymbol{y}}$ if

$$
\overline{\boldsymbol{y}} \in \overline{\operatorname{int}\left\{\boldsymbol{y} \in O ; \boldsymbol{H}^{(i)}(\boldsymbol{y})=\boldsymbol{H}(\boldsymbol{y})\right\}} .
$$

The set of indices of active and essentially active selection functions of $\boldsymbol{H}$ at $\overline{\boldsymbol{y}}$ are denoted by $\mathcal{I}_{\boldsymbol{H}}(\overline{\boldsymbol{y}})$ and $\mathcal{I}_{\boldsymbol{H}}^{e}(\overline{\boldsymbol{y}})$, respectively. 
Due to continuity, selection functions can be chosen at every point $\overline{\boldsymbol{y}}$ so that they are all active there. A composition of two $P C^{1}$-functions is again a $P C^{1}$-function and a vector-valued function is a $P C^{1}$-function if and only if all its component functions are $P C^{1}$. One can also show that every $P C^{1}$-function is locally Lipschitz-continuous and Bouligand-differentiable (B-differentiable). Let us recall that the B-derivative of $\boldsymbol{H}$ at $\boldsymbol{y}$ in the direction $\boldsymbol{z}$ is the directional derivative $\boldsymbol{H}^{\prime}(\boldsymbol{y} ; \boldsymbol{z})$ that satisfies:

$$
\lim _{\boldsymbol{z} \rightarrow \mathbf{0}} \frac{\left\|\boldsymbol{H}(\boldsymbol{y}+\boldsymbol{z})-\boldsymbol{H}(\boldsymbol{y})-\boldsymbol{H}^{\prime}(\boldsymbol{y} ; \boldsymbol{z})\right\|}{\|\boldsymbol{z}\|}=0 .
$$

The following chain rule holds for B-derivatives:

Proposition 1. Let $\boldsymbol{G}: \mathbb{R}^{M} \rightarrow \mathbb{R}^{N}$ and $\boldsymbol{H}: \mathbb{R}^{N} \rightarrow \mathbb{R}^{P}$ be locally Lipschitz-continuous and B-differentiable at $\boldsymbol{y}$ and $\boldsymbol{G}(\boldsymbol{y})$, respectively. Then the composite function $\boldsymbol{H} \circ \boldsymbol{G}$ is $B$-differentiable at $\boldsymbol{y}$ and

$$
(\boldsymbol{H} \circ \boldsymbol{G})^{\prime}(\boldsymbol{y} ; \boldsymbol{z})=\boldsymbol{H}^{\prime}\left(\boldsymbol{G}(\boldsymbol{y}) ; \boldsymbol{G}^{\prime}(\boldsymbol{y} ; \boldsymbol{z})\right) .
$$

If the inner function is a function of real variable, one can also prove:

Proposition 2. If $\boldsymbol{G}:\left[s_{0}, s_{0}+\delta\right) \rightarrow \mathbb{R}^{N}$ is differentiable at $s_{0}$ from the right and $\boldsymbol{H}: \mathbb{R}^{N} \rightarrow$ $\mathbb{R}^{P}$ is locally Lipschitz-continuous and B-differentiable at $\boldsymbol{G}(\boldsymbol{y})$, then $\boldsymbol{H} \circ \boldsymbol{G}$ has a righthand derivative at $s_{0}$ and

$$
(\boldsymbol{H} \circ \boldsymbol{G})^{\prime}\left(s_{0}\right)=\boldsymbol{H}^{\prime}\left(\boldsymbol{G}\left(s_{0}\right) ; \boldsymbol{G}^{\prime}\left(s_{0}\right)\right) .
$$

The following definition introduces two important notions for $P C^{1}$-functions.

Definition 2. Let $\boldsymbol{F}: \mathbb{R}^{N} \times \mathbb{R}^{M} \rightarrow \mathbb{R}^{N}$ be a $P C^{1}$-function with essentially active selection functions $\boldsymbol{F}^{(1)}, \ldots, \boldsymbol{F}^{(n)}$ at $(\overline{\boldsymbol{x}}, \overline{\boldsymbol{y}})$.

(i) The function $\boldsymbol{F}$ is called coherently oriented with respect to $\boldsymbol{x}$ at $(\overline{\boldsymbol{x}}, \overline{\boldsymbol{y}})$ iff the partial gradients $\boldsymbol{\nabla}_{x} \boldsymbol{F}^{(i)}(\overline{\boldsymbol{x}}, \overline{\boldsymbol{y}}), i=1, \ldots, n$, have the same non-vanishing determinantal sign.

(ii) The function $\boldsymbol{F}$ is completely coherently oriented with respect to $\boldsymbol{x}$ at $(\overline{\boldsymbol{x}}, \overline{\boldsymbol{y}})$ if all $N \times N$ matrices of the form

$$
\left(\begin{array}{c}
\nabla_{\boldsymbol{x}}^{\top} F_{1}^{\left(i_{1}\right)}(\overline{\boldsymbol{x}}, \overline{\boldsymbol{y}}) \\
\nabla_{\boldsymbol{x}}^{\top} F_{2}^{\left(i_{2}\right)}(\overline{\boldsymbol{x}}, \overline{\boldsymbol{y}}) \\
\vdots \\
\nabla_{\boldsymbol{x}}^{\top} F_{N}^{\left(i_{N}\right)}(\overline{\boldsymbol{x}}, \overline{\boldsymbol{y}})
\end{array}\right),
$$

where $i_{1}, i_{2}, \ldots i_{N} \in\{1, \ldots n\}$, have the same non-vanishing determinantal sign.

We say that $\boldsymbol{F}$ is (completely) coherently oriented with respect to $\boldsymbol{x}$ if it is (completely) coherently oriented with respect to $\boldsymbol{x}$ on $\mathbb{R}^{N} \times \mathbb{R}^{M}$. Besides, if $M=0$, that is, if the dimensions of the preimage and image space of $\boldsymbol{F}$ coincide, then we simply speak of (complete) coherent orientation of $\boldsymbol{F}$. 
A special case of a $P C^{1}$-function is a piecewise-linear function. It is a continuous function whose selection functions are linear, that is, of the form $\boldsymbol{y} \mapsto \boldsymbol{A}^{(i)} \boldsymbol{y}$ for some matrices $\boldsymbol{A}^{(i)}$. It is known that the B-derivative $\boldsymbol{H}^{\prime}\left(\boldsymbol{y} ;\right.$.) of a $P C^{1}$-function $\boldsymbol{H}$ is a piecewise-linear function. In this case, $\boldsymbol{H}^{\prime}(\boldsymbol{y} ;$.) is even Lipschitz-continuous and

$$
\boldsymbol{H}^{\prime}(\boldsymbol{y} ; \boldsymbol{z}) \in\left\{\boldsymbol{\nabla} \boldsymbol{H}^{(i)}(\boldsymbol{y}) \boldsymbol{z} ; i \in \mathcal{I}_{\boldsymbol{H}}^{e}(\boldsymbol{y})\right\}
$$

We conclude this preparatory part with two useful results.

Proposition 3. A coherently oriented piecewise-linear function is surjective. If it is in addition completely coherently oriented, it is a homeomorphism.

Proposition 4. Let $\boldsymbol{F}: \mathbb{R}^{N} \times \mathbb{R}^{M} \rightarrow \mathbb{R}^{N}$ be a $P C^{1}$-function. If $\boldsymbol{F}$ is completely coherently oriented with respect to $\boldsymbol{x}$ at a zero $(\overline{\boldsymbol{x}}, \overline{\boldsymbol{y}})$ of $\boldsymbol{F}$, then the equation $\boldsymbol{F}(\boldsymbol{x}, \boldsymbol{y})=\mathbf{0}$ determines a unique implicit $P C^{1}$-function $\boldsymbol{x}(\boldsymbol{y})$ in a neighbourhood of $(\overline{\boldsymbol{x}}, \overline{\boldsymbol{y}})$.

\section{Abstract Continuation Problem}

Let us consider a nonlinear evolution mathematical model whose spatial semi-discretisation leads to a problem of the type:

$$
\left.\begin{array}{l}
\text { Find } \boldsymbol{x}:[0, T] \rightarrow \mathbb{R}^{N} \text { such that } \\
\boldsymbol{G}(\boldsymbol{x}(t), \dot{\boldsymbol{x}}(t))=\boldsymbol{F}(t, \boldsymbol{x}(t)) \text { in }(0, T), \quad \boldsymbol{x}(0)=\boldsymbol{x}^{0}
\end{array}\right\}
$$

with $N \in \mathbb{N}, T>0, \boldsymbol{G}: \mathbb{R}^{N} \times \mathbb{R}^{N} \rightarrow \mathbb{R}^{N}, \boldsymbol{F}: \mathbb{R} \times \mathbb{R}^{N} \rightarrow \mathbb{R}^{N}$ and $\boldsymbol{x}_{0} \in \mathbb{R}^{N}$ given. Here, $\dot{\boldsymbol{x}} \equiv \mathrm{d} \boldsymbol{x} / \mathrm{d} t$ stands for the time derivative of $\boldsymbol{x}$.

To illustrate the idea how to construct the announced continuation problem for a particular time discretisation, we divide the interval $[0, T]$ into $n_{T}$ sub-intervals $\left[t_{k}, t_{k+1}\right]$, $k=0, \ldots, n_{T}-1$, and use the backward difference

$$
\dot{\boldsymbol{x}}\left(t_{k+1}\right) \approx \frac{\boldsymbol{x}\left(t_{k+1}\right)-\boldsymbol{x}\left(t_{k}\right)}{t_{k+1}-t_{k}} .
$$

In this way, we obtain a sequence of incremental problems for $k=0, \ldots, n_{T}-1$ :

$$
\left.\begin{array}{l}
\text { Find } \boldsymbol{x}^{k+1} \in \mathbb{R}^{N} \text { such that } \\
\boldsymbol{G}\left(\boldsymbol{x}^{k+1}, \frac{\boldsymbol{x}^{k+1}-\boldsymbol{x}^{k}}{t_{k+1}-t_{k}}\right)=\boldsymbol{F}\left(t_{k+1}, \boldsymbol{x}^{k+1}\right) .
\end{array}\right\}
$$

Now, let $k \geq 1$ be fixed. We introduce the continuation problem with an additional scalar parameter $\gamma$ as follows:

$$
\left.\begin{array}{l}
\text { Find }(\gamma, \boldsymbol{x}) \in \mathbb{R} \times \mathbb{R}^{N} \text { such that } \\
\boldsymbol{H}(\gamma, \boldsymbol{x})=\mathbf{0},
\end{array}\right\}
$$


where $\boldsymbol{H}: \mathbb{R} \times \mathbb{R}^{N} \rightarrow \mathbb{R}^{N}$ is defined as

$$
\boldsymbol{H}(\gamma, \boldsymbol{x}):=\boldsymbol{G}\left(\boldsymbol{x}, \frac{\boldsymbol{x}-\boldsymbol{x}^{k}}{t_{k+1}-t_{k}}+(1-\gamma) \frac{\boldsymbol{x}^{k}-\boldsymbol{x}^{k-1}}{t_{k}-t_{k-1}}\right)-\left(\gamma \boldsymbol{F}\left(t_{k+1}, \boldsymbol{x}\right)+(1-\gamma) \boldsymbol{F}\left(t_{k}, \boldsymbol{x}\right)\right) \text {. }
$$

It is readily seen that any $\boldsymbol{x}^{k}$ solving $\left(\mathscr{P}_{k}\right)$ satisfies $\boldsymbol{H}\left(0, \boldsymbol{x}^{k}\right)=\mathbf{0}$ and $\boldsymbol{x}$ solves $\left(\mathscr{P}_{k+1}\right)$ if and only if $\boldsymbol{H}(1, \boldsymbol{x})=\mathbf{0}$. This furnishes us with the following method for finding solutions of $\left(\mathscr{P}_{k+1}\right)$ provided that we are able to continue solutions of $(\mathscr{P})$ : starting with $\left(0, \boldsymbol{x}^{k}\right)$ as the initial point, any couple $(\gamma, \boldsymbol{x})$ with $\gamma=1$ found during the continuation gives $\boldsymbol{x}$ solving Problem $\left(\mathscr{P}_{k+1}\right)$.

Observe that the passage from $\gamma=0$ to $\gamma=1$ corresponds to a transition of the right-hand side of Problem (1) from the values on the $k$ th time level to the values on the $(k+1)$ st one. Thus, $(\mathscr{P})$ may be viewed as a problem with parametrised right-hand side. But one should be aware that $\gamma$ does not play the exact role of time. The time increment is keeping fixed!

Let us also note that the term $(1-\gamma)\left(\boldsymbol{x}^{k}-\boldsymbol{x}^{k-1}\right) /\left(t_{k}-t_{k-1}\right)$ in the definition of $\boldsymbol{H}$ adds a kind of memory into $(\mathscr{P})$ when starting from $\left(0, \boldsymbol{x}^{k}\right)$. Hence, the $\boldsymbol{x}$-parts of solutions of $(\mathscr{P})$ can be expected to be consistent with $\boldsymbol{x}^{k}$ (see also Example 3 and the discussion preceding to it in Section 3).

Remark 1. The backward difference used in the time discretisation corresponds to the backward Euler method. Nevertheless, our approach can be easily modified for other classical time discretisation schemes like the $\theta$-method or midpoint rule. Indeed, when using any time-stepping scheme, one can always introduce a parameter $\gamma$ so that problems on successive time levels are recovered for $\gamma$ equal to 0 and 1 , respectively. Clearly, our approach can be applied also to any second-order evolution problem after rewriting it as a system of the first order.

In the remaining part of this section, we shall suppose that the function $\boldsymbol{H}$ in Problem $(\mathscr{P})$ is an arbitrary $P C^{1}$-function. This is typically so if both $\boldsymbol{F}$ and $\boldsymbol{G}$ are $P C^{1}$.

\subsection{Analysis}

One would expect that the solution set of $\boldsymbol{H}(\gamma, \boldsymbol{x})=\mathbf{0}$ could be described as a curve. We shall show that under assumptions of an appropriate implicit function theorem, this is indeed, at least locally, the case. In particular, we shall use the notion of a completely coherently oriented function introduced in Subsection 1.1.

Let $\boldsymbol{H}$ be a $P C^{1}$-function and $(\bar{\gamma}, \overline{\boldsymbol{x}}) \in \mathbb{R} \times \mathbb{R}^{N}$ be such that

(i) $\boldsymbol{H}(\bar{\gamma}, \overline{\boldsymbol{x}})=\mathbf{0}$;
(ii) $\boldsymbol{H}$ is completely coherently oriented with respect to $\boldsymbol{x}$ at $(\bar{\gamma}, \overline{\boldsymbol{x}})$.

Then it follows from Proposition 4 that the equation $\boldsymbol{H}(\gamma, \boldsymbol{x})=\mathbf{0}$ determines a unique implicit $P C^{1}$-function $\boldsymbol{x}(\gamma)$ in a neighbourhood of $(\bar{\gamma}, \overline{\boldsymbol{x}})$ with $\boldsymbol{x}(\bar{\gamma})=\overline{\boldsymbol{x}}$. Defining a curve c $: J \rightarrow \mathbb{R} \times \mathbb{R}^{N}$ on the corresponding neighbourhood $J$ of $\bar{\gamma}$ as

$$
\boldsymbol{c}: s \mapsto(s, \boldsymbol{x}(s)),
$$


we arrive immediately at the following proposition (let us point out that we do not consider $s$ to be the arc-length parameter here and in what follows - it is not necessary to use parametrisation by arc-length in our exposition):

Proposition 5. Let $\boldsymbol{H}: \mathbb{R} \times \mathbb{R}^{N} \rightarrow \mathbb{R}^{N}$ be a $P C^{1}$-function and $(\bar{\gamma}, \overline{\boldsymbol{x}}) \in \mathbb{R} \times \mathbb{R}^{N}$ satisfy (2). Then there exist an open interval $J$ and a $P C^{1}$-curve $\boldsymbol{c}: J \rightarrow \mathbb{R} \times \mathbb{R}^{N}$ such that

$$
\text { (j) } \exists \bar{s} \in J: \quad \boldsymbol{c}(\bar{s})=(\bar{\gamma}, \overline{\boldsymbol{x}}) ; \quad(\mathrm{jj}) \forall s \in J: \quad \boldsymbol{H}(\boldsymbol{c}(s))=\mathbf{0} .
$$

Moreover, the solution set of $\boldsymbol{H}(\gamma, \boldsymbol{x})=\mathbf{0}$ coincides with the image of $\boldsymbol{c}$ in a neighbourhood of $(\bar{\gamma}, \overline{\boldsymbol{x}})$.

Keeping this result in mind, we shall suppose in the following that we are given a zero $(\bar{\gamma}, \overline{\boldsymbol{x}})$ of $\boldsymbol{H}$ and there exists a $P C^{1}$-curve $\boldsymbol{c}: s \mapsto \boldsymbol{c}(s)=(\gamma(s), \boldsymbol{x}(s))$ satisfying (3). Since any $P C^{1}$-function is directionally differentiable, we can compute the right-hand derivative of $(3)(\mathrm{jj})$ at $\bar{s}$ according to Proposition 2 :

$$
\boldsymbol{H}^{\prime}(\boldsymbol{c}(\bar{s}))=\boldsymbol{H}^{\prime}\left(\boldsymbol{c}(\bar{s}) ; \boldsymbol{c}^{\prime}(\bar{s})\right)=\boldsymbol{H}^{\prime}\left((\bar{\gamma}, \overline{\boldsymbol{x}}) ;\left(\gamma^{\prime}(\bar{s}), \boldsymbol{x}^{\prime}(\bar{s})\right)\right)=\mathbf{0}
$$

Therefore, if $\boldsymbol{c}$ is a curve passing through $(\bar{\gamma}, \overline{\boldsymbol{x}})$ whose course we do not know completely, we can recover its tangent behaviour at $(\bar{\gamma}, \overline{\boldsymbol{x}})$ by solving the problem:

$$
\left.\begin{array}{l}
\text { Find }\left(\gamma^{\prime}, \boldsymbol{x}^{\prime}\right) \in \mathbb{R} \times \mathbb{R}^{N} \text { such that } \\
\boldsymbol{H}^{\prime}\left((\bar{\gamma}, \overline{\boldsymbol{x}}) ;\left(\gamma^{\prime}, \boldsymbol{x}^{\prime}\right)\right)=\mathbf{0} .
\end{array}\right\}
$$

By analogy with continuation of smooth curves, we call this problem a first-order system.

The derivative $\boldsymbol{H}^{\prime}\left((\bar{\gamma}, \overline{\boldsymbol{x}}) ;\left(\gamma^{\prime}, \boldsymbol{x}^{\prime}\right)\right)$ is a piecewise-linear function of $\left(\gamma^{\prime}, \boldsymbol{x}^{\prime}\right)$ for any $P C^{1}$ function $\boldsymbol{H}$. Invoking theory of piecewise-linear functions, we have results of existence and uniqueness of solutions of $\left(\mathscr{P}^{\prime}\right)$.

Proposition 6. Let $\boldsymbol{H}: \mathbb{R} \times \mathbb{R}^{N} \rightarrow \mathbb{R}^{N}$ be a PC $C^{1}$-function that is coherently oriented with respect to $\boldsymbol{x}$ at $(\bar{\gamma}, \overline{\boldsymbol{x}})$. Then for any $\gamma^{\prime} \in \mathbb{R}$, there exists at least one $\boldsymbol{x}^{\prime} \in \mathbb{R}^{N}$ such that $\left(\gamma^{\prime}, \boldsymbol{x}^{\prime}\right)$ solves $\left(\mathscr{P}^{\prime}\right)$. If $\boldsymbol{H}$ is even completely coherently oriented with respect to $\boldsymbol{x}$ at $(\bar{\gamma}, \overline{\boldsymbol{x}})$, the vector $\boldsymbol{x}^{\prime}$ is determined uniquely.

Proof. Under the imposed assumptions, the mapping $\tilde{\boldsymbol{H}}: \mathbb{R} \times \mathbb{R}^{N} \rightarrow \mathbb{R} \times \mathbb{R}^{N}$ introduced as

$$
\tilde{\boldsymbol{H}}:\left(\gamma^{\prime}, \boldsymbol{x}^{\prime}\right) \mapsto\left(\begin{array}{c}
\boldsymbol{H}^{\prime}\left((\bar{\gamma}, \overline{\boldsymbol{x}}) ;\left(\gamma^{\prime}, \boldsymbol{x}^{\prime}\right)\right) \\
\gamma^{\prime}
\end{array}\right)
$$

is piecewise-linear and (completely) coherently oriented and the assertions follow directly from Proposition 3.

For other possible criteria guaranteeing unique solvability of $\left(\mathscr{P}^{\prime}\right)$, see [24, Section 4], [1] and the references therein. 
So far, we have shown that any one-sided tangent vector of a solution curve of $(\mathscr{P})$ is a solution of the first-order system $\left(\mathscr{P}^{\prime}\right)$. Now, we shall deal with the converse: Having a solution $\left(\gamma^{\prime}, \boldsymbol{x}^{\prime}\right)$ of $\left(\mathscr{P}^{\prime}\right)$ at our disposal, we shall give conditions under which it corresponds to a tangent to a solution branch of $(\mathscr{P})$ emanating from the corresponding point. We shall obtain even uniqueness of such a solution branch.

The first result complements Proposition 5.

Theorem 1. Let the assumptions of Proposition 5 be fulfilled and $\left(\gamma^{\prime}, \boldsymbol{x}^{\prime}\right) \in \mathbb{R} \times \mathbb{R}^{N}$ solve $\left(\mathscr{P}^{\prime}\right)$. Then there are $\delta>0$ and a $P C^{1}$-curve $\boldsymbol{c}:[\bar{s}, \bar{s}+\delta) \rightarrow \mathbb{R} \times \mathbb{R}^{N}$ such that

$$
(\mathrm{j}) \boldsymbol{c}(\bar{s})=(\bar{\gamma}, \overline{\boldsymbol{x}}) ; \quad(\mathrm{jj}) \forall s \in[\bar{s}, \bar{s}+\delta): \boldsymbol{H}(\boldsymbol{c}(s))=\mathbf{0} ; \quad(\mathrm{jjj}) \boldsymbol{c}^{\prime}(\bar{s})=\left(\gamma^{\prime}, \boldsymbol{x}^{\prime}\right) .
$$

Moreover, if $\left(\gamma^{\prime}, \boldsymbol{x}^{\prime}\right) \neq(0, \mathbf{0})$, the image of any other curve $\tilde{\boldsymbol{c}}:[\bar{s}, \bar{s}+\tilde{\delta}) \rightarrow \mathbb{R} \times \mathbb{R}^{N}$ with $\tilde{\delta}>0$ and such that

$$
(\widetilde{\mathrm{j}}) \tilde{\boldsymbol{c}}(\bar{s})=(\bar{\gamma}, \overline{\boldsymbol{x}}) ; \quad(\widetilde{\mathrm{jj}}) \forall s \in[\bar{s}, \bar{s}+\tilde{\delta}): \boldsymbol{H}(\tilde{\boldsymbol{c}}(s))=\mathbf{0} ; \quad(\widetilde{\mathrm{jjj}}) \tilde{\boldsymbol{c}}^{\prime}(\bar{s}) \in \bigcup_{r>0} r\left(\gamma^{\prime}, \boldsymbol{x}^{\prime}\right)
$$

coincides with the image of $\boldsymbol{c}$ in a neighbourhood of $(\bar{\gamma}, \overline{\boldsymbol{x}})$.

Proof. As we already know, $\boldsymbol{H}(\gamma, \boldsymbol{x})=\mathbf{0}$ determines a unique implicit $P C^{1}$-function $\boldsymbol{x}(\gamma)$ in a neighbourhood of $(\bar{\gamma}, \overline{\boldsymbol{x}})$. Let us define $\boldsymbol{c}$ as

$$
\boldsymbol{c}: s \mapsto\left(\gamma^{\prime}(s-\bar{s})+\bar{\gamma}, \boldsymbol{x}\left(\gamma^{\prime}(s-\bar{s})+\bar{\gamma}\right)\right) .
$$

Then $(4)(\mathrm{j})$ and $(\mathrm{jj})$ are clearly satisfied for sufficiently small $\delta>0$ and

$$
\boldsymbol{c}^{\prime}(\bar{s})=\left(\gamma^{\prime}, \gamma^{\prime} \boldsymbol{x}^{\prime}(\bar{\gamma})\right)
$$

From the derivation of $\left(\mathscr{P}^{\prime}\right)$, we know that $\left(\gamma^{\prime}, \gamma^{\prime} \boldsymbol{x}^{\prime}(\bar{\gamma})\right)$ belongs to its solutions and the uniqueness result of Proposition 6 implies that

$$
\gamma^{\prime} \boldsymbol{x}^{\prime}(\bar{\gamma})=\boldsymbol{x}^{\prime}
$$

which completes (4). The second part of the assertion follows from the uniqueness of the implicit function $\boldsymbol{x}(\gamma)$ in a neighbourhood of $(\bar{\gamma}, \overline{\boldsymbol{x}})$.

Due to the implicit function theorem used in the proof, the previous theorem can be applied only to cases where exactly two one-sided solution branches $s \mapsto(\gamma(s), \boldsymbol{x}(s))$ emanate from $(\bar{\gamma}, \overline{\boldsymbol{x}})$ - one with $\gamma$ increasing and the other one with $\gamma$ decreasing. The next theorem covers also situations with more solution branches.

Theorem 2. Let $\boldsymbol{H}: \mathbb{R} \times \mathbb{R}^{N} \rightarrow \mathbb{R}^{N}$ be a $P C^{1}$-function and $(\bar{\gamma}, \overline{\boldsymbol{x}})$, $\left(\gamma^{\prime}, \boldsymbol{x}^{\prime}\right) \in \mathbb{R} \times \mathbb{R}^{N}$ satisfy $\boldsymbol{H}(\bar{\gamma}, \overline{\boldsymbol{x}})=\mathbf{0}$ and $\boldsymbol{H}^{\prime}\left((\bar{\gamma}, \overline{\boldsymbol{x}}) ;\left(\gamma^{\prime}, \boldsymbol{x}^{\prime}\right)\right)=\mathbf{0}$. If $\left\{\boldsymbol{H}^{(i)}\right\}_{i \in \mathcal{I}}$ is a family of $C^{1}$ selection functions of $\boldsymbol{H}$ at $(\bar{\gamma}, \overline{\boldsymbol{x}})$ and there exists $i_{0} \in \mathcal{I}$ such that

(i) $\boldsymbol{H}^{\left(i_{0}\right)}(\bar{\gamma}, \overline{\boldsymbol{x}})=\boldsymbol{H}(\bar{\gamma}, \overline{\boldsymbol{x}})$;

(ii) $\left.\boldsymbol{\nabla} \boldsymbol{H}^{\left(i_{0}\right)}(\bar{\gamma}, \overline{\boldsymbol{x}})\left(\gamma^{\prime}, \boldsymbol{x}^{\prime}\right)=\boldsymbol{H}^{\prime}\left((\bar{\gamma}, \overline{\boldsymbol{x}}) ;\left(\gamma^{\prime}, \boldsymbol{x}^{\prime}\right)\right) \neq \boldsymbol{\nabla} \boldsymbol{H}^{(i)}(\bar{\gamma}, \overline{\boldsymbol{x}})\left(\gamma^{\prime}, \boldsymbol{x}^{\prime}\right), \forall i \neq i_{0} ;\right\}$
(iii) $\boldsymbol{\nabla}_{\boldsymbol{x}} \boldsymbol{H}^{\left(i_{0}\right)}(\bar{\gamma}, \overline{\boldsymbol{x}})$ is non-singular,

then all the conclusions of Theorem 1 hold and the solution curve $\boldsymbol{c}$ is even of the class $C^{1}$. 
Proof. By the classical implicit function theorem, the equation $\boldsymbol{H}^{\left(i_{0}\right)}(\gamma, \boldsymbol{x})=\mathbf{0}$ determines a unique implicit function $\boldsymbol{x}(\gamma)$ around $(\bar{\gamma}, \overline{\boldsymbol{x}})$. In this case, $\boldsymbol{x}(\gamma)$ is continuously differentiable and its (two-sided) derivative is:

$$
\boldsymbol{x}^{\prime}(\bar{\gamma})=-\left(\boldsymbol{\nabla}_{\boldsymbol{x}} \boldsymbol{H}^{\left(i_{0}\right)}(\bar{\gamma}, \overline{\boldsymbol{x}})\right)^{-1} \boldsymbol{\nabla}_{\gamma} \boldsymbol{H}^{\left(i_{0}\right)}(\bar{\gamma}, \overline{\boldsymbol{x}}) .
$$

Let us define a curve $\boldsymbol{c}$ by

$$
\boldsymbol{c}: s \mapsto\left(\gamma^{\prime}(s-\bar{s})+\bar{\gamma}, \boldsymbol{x}\left(\gamma^{\prime}(s-\bar{s})+\bar{\gamma}\right)\right)
$$

Then (4)(j) clearly holds. By (6)(ii) and (iii),

$$
\begin{aligned}
\boldsymbol{\nabla} \boldsymbol{H}^{\left(i_{0}\right)}(\bar{\gamma}, \overline{\boldsymbol{x}})\left(\gamma^{\prime}, \boldsymbol{x}^{\prime}\right) & =\boldsymbol{\nabla}_{\gamma} \boldsymbol{H}^{\left(i_{0}\right)}(\bar{\gamma}, \overline{\boldsymbol{x}}) \gamma^{\prime}+\boldsymbol{\nabla}_{\boldsymbol{x}} \boldsymbol{H}^{\left(i_{0}\right)}(\bar{\gamma}, \overline{\boldsymbol{x}}) \boldsymbol{x}^{\prime}=\boldsymbol{H}^{\prime}\left((\bar{\gamma}, \overline{\boldsymbol{x}}) ;\left(\gamma^{\prime}, \boldsymbol{x}^{\prime}\right)\right)=\mathbf{0}, \\
\boldsymbol{x}^{\prime} & =-\gamma^{\prime}\left(\boldsymbol{\nabla}_{\boldsymbol{x}} \boldsymbol{H}^{\left(i_{0}\right)}(\bar{\gamma}, \overline{\boldsymbol{x}})\right)^{-1} \boldsymbol{\nabla}_{\gamma} \boldsymbol{H}^{\left(i_{0}\right)}(\bar{\gamma}, \overline{\boldsymbol{x}}) .
\end{aligned}
$$

This combined with (7) yields

$$
\boldsymbol{c}^{\prime}(\bar{s})=\left(\gamma^{\prime}, \gamma^{\prime} \boldsymbol{x}^{\prime}(\bar{\gamma})\right)=\left(\gamma^{\prime},-\gamma^{\prime}\left(\boldsymbol{\nabla}_{\boldsymbol{x}} \boldsymbol{H}^{\left(i_{0}\right)}(\bar{\gamma}, \overline{\boldsymbol{x}})\right)^{-1} \boldsymbol{\nabla}_{\gamma} \boldsymbol{H}^{\left(i_{0}\right)}(\bar{\gamma}, \overline{\boldsymbol{x}})\right)=\left(\gamma^{\prime}, \boldsymbol{x}^{\prime}\right),
$$

that is, (4)(jjj) is fulfilled, as well. Moreover, $\boldsymbol{H}^{\left(i_{0}\right)}(\boldsymbol{c}(s))=\mathbf{0}$, and to prove $(4)(\mathrm{jj})$, we have to show:

$$
\boldsymbol{H}(\boldsymbol{c}(s))=\boldsymbol{H}^{\left(i_{0}\right)}(\boldsymbol{c}(s)) \text { for } s \geq \bar{s}, s \text { close to } \bar{s} .
$$

Hereafter, we shall suppose that

$$
\forall i \in \mathcal{I}: \boldsymbol{H}^{(i)}(\bar{\gamma}, \overline{\boldsymbol{x}})=\boldsymbol{H}(\bar{\gamma}, \overline{\boldsymbol{x}})
$$

(the other indices may be omitted from $\mathcal{I}$ by virtue of the continuity of $\boldsymbol{H}$ ).

Making use of (6)(ii), one can find for any $i \neq i_{0}$ an index $j(i)$ such that

$$
\nabla^{\top} H_{j(i)}^{(i)}(\bar{\gamma}, \overline{\boldsymbol{x}})\left(\gamma^{\prime}, \boldsymbol{x}^{\prime}\right) \neq \nabla^{\top} H_{j(i)}^{\left(i_{0}\right)}(\bar{\gamma}, \overline{\boldsymbol{x}})\left(\gamma^{\prime}, \boldsymbol{x}^{\prime}\right)
$$

which ensures $\epsilon$ defined by

$$
\epsilon:=\min _{i \neq i_{0}}\left|\left(\nabla^{\top} H_{j(i)}^{(i)}(\bar{\gamma}, \overline{\boldsymbol{x}})-\nabla^{\top} H_{j(i)}^{\left(i_{0}\right)}(\bar{\gamma}, \overline{\boldsymbol{x}})\right)\left(\gamma^{\prime}, \boldsymbol{x}^{\prime}\right)\right|
$$

to be positive. Continuous differentiability of $\boldsymbol{H}^{(i)}$ implies that there is $\bar{\delta}>0$ such that

$$
\forall i \neq i_{0} \forall(\gamma, \boldsymbol{x}) \in B((\bar{\gamma}, \overline{\boldsymbol{x}}), \bar{\delta}):\left|\left(\nabla^{\top} H_{j(i)}^{(i)}(\gamma, \boldsymbol{x})-\nabla^{\top} H_{j(i)}^{\left(i_{0}\right)}(\gamma, \boldsymbol{x})\right)\left(\gamma^{\prime}, \boldsymbol{x}^{\prime}\right)\right| \geq \frac{\epsilon}{2}
$$

and consequently

$$
\begin{aligned}
& \forall i \neq i_{0} \forall(\gamma, \boldsymbol{x}) \in B((\bar{\gamma}, \overline{\boldsymbol{x}}), \bar{\delta}) \forall(u, \boldsymbol{v}) \in B\left(\left(\gamma^{\prime}, \boldsymbol{x}^{\prime}\right), \delta^{\prime}\right): \\
& \left|\left(\nabla^{\top} H_{j(i)}^{(i)}(\gamma, \boldsymbol{x})-\nabla^{\top} H_{j(i)}^{\left(i_{0}\right)}(\gamma, \boldsymbol{x})\right)(u, \boldsymbol{v})\right| \\
& \geq \mid \\
& \left.\quad \mid \nabla^{\top} H_{j(i)}^{(i)}(\gamma, \boldsymbol{x})-\nabla^{\top} H_{j(i)}^{\left(i_{0}\right)}(\gamma, \boldsymbol{x})\right)\left(\gamma^{\prime}, \boldsymbol{x}^{\prime}\right) \mid \\
& \quad-\left|\left(\nabla^{\top} H_{j(i)}^{(i)}(\gamma, \boldsymbol{x})-\nabla^{\top} H_{j(i)}^{\left(i_{0}\right)}(\gamma, \boldsymbol{x})\right)\left(u-\gamma^{\prime}, \boldsymbol{v}-\boldsymbol{x}^{\prime}\right)\right| \\
& \geq \frac{\epsilon}{2}-\left\|\nabla H_{j(i)}^{(i)}(\gamma, \boldsymbol{x})-\nabla H_{j(i)}^{\left(i_{0}\right)}(\gamma, \boldsymbol{x})\right\|\left\|\left(u-\gamma^{\prime}, \boldsymbol{v}-\boldsymbol{x}^{\prime}\right)\right\|>0
\end{aligned}
$$


for some $\delta^{\prime}>0$ by boundedness of $\nabla H_{j(i)}^{(i)}(\gamma, \boldsymbol{x})-\nabla H_{j(i)}^{\left(i_{0}\right)}(\gamma, \boldsymbol{x})$. Therefore, there is $\bar{r}>0$ such that

$$
\begin{aligned}
\forall i \neq i_{0} \forall(u, \boldsymbol{v}) \in & B\left(\left(\gamma^{\prime}, \boldsymbol{x}^{\prime}\right), \delta^{\prime}\right) \forall r \in[0, \bar{r}): \\
& \left|\left(\nabla^{\top} H_{j(i)}^{(i)}(\bar{\gamma}+r u, \overline{\boldsymbol{x}}+r \boldsymbol{v})-\nabla^{\top} H_{j(i)}^{\left(i_{0}\right)}(\bar{\gamma}+r u, \overline{\boldsymbol{x}}+r \boldsymbol{v})\right)(u, \boldsymbol{v})\right|>0
\end{aligned}
$$

and due to $(6)\left(\right.$ ii) and the continuity of $\boldsymbol{H}^{\prime}((\bar{\gamma}, \overline{\boldsymbol{x}}) ;$.$) ,$

$$
\begin{aligned}
& \forall i \neq i_{0} \forall(u, \boldsymbol{v}) \in B\left(\left(\gamma^{\prime}, \boldsymbol{x}^{\prime}\right), \delta^{\prime}\right): \\
& \boldsymbol{H}^{\prime}((\bar{\gamma}, \overline{\boldsymbol{x}}) ;(u, \boldsymbol{v}))=\nabla \boldsymbol{H}^{\left(i_{0}\right)}(\bar{\gamma}, \overline{\boldsymbol{x}})(u, \boldsymbol{v}) \neq \boldsymbol{\nabla} \boldsymbol{H}^{(i)}(\bar{\gamma}, \overline{\boldsymbol{x}})(u, \boldsymbol{v}) .
\end{aligned}
$$

Now, let us take $(u, \boldsymbol{v}) \in B\left(\left(\gamma^{\prime}, \boldsymbol{x}^{\prime}\right), \delta^{\prime}\right)$ arbitrary but fixed and define $\boldsymbol{\phi}, \boldsymbol{\phi}^{(i)}: \mathbb{R} \rightarrow \mathbb{R}^{N}$ by

$$
\boldsymbol{\phi}: r \mapsto \boldsymbol{H}(\bar{\gamma}+r u, \overline{\boldsymbol{x}}+r \boldsymbol{v}), \quad \boldsymbol{\phi}^{(i)}: r \mapsto \boldsymbol{H}^{(i)}(\bar{\gamma}+r u, \overline{\boldsymbol{x}}+r \boldsymbol{v})
$$

Clearly,

$$
\phi^{\prime}(r)=\boldsymbol{H}^{\prime}((\bar{\gamma}+r u, \overline{\boldsymbol{x}}+r \boldsymbol{v}) ;(u, \boldsymbol{v})), \quad\left(\phi^{(i)}\right)^{\prime}(r)=\boldsymbol{\nabla} \boldsymbol{H}^{(i)}(\bar{\gamma}+r u, \overline{\boldsymbol{x}}+r \boldsymbol{v})(u, \boldsymbol{v})
$$

and by virtue of (10), (11) and (12),

$$
\begin{gathered}
\forall i \in \mathcal{I}: \quad \phi^{(i)}(0)=\phi(0), \\
\forall i \neq i_{0} \forall r \in[0, \bar{r}): \quad\left|\left(\phi_{j(i)}^{(i)}\right)^{\prime}(r)-\left(\phi_{j(i)}^{\left(i_{0}\right)}\right)^{\prime}(r)\right|>0, \\
\forall i \neq i_{0}: \quad \phi^{\prime}(0) \neq\left(\phi^{(i)}\right)^{\prime}(0) .
\end{gathered}
$$

As $\phi^{(i)}$ are of the class $C^{1}$, we obtain

$$
\begin{aligned}
\forall i \neq i_{0} \forall r \in(0, \bar{r}):\left|\phi_{j(i)}^{(i)}(r)-\phi_{j(i)}^{\left(i_{0}\right)}(r)\right| & =\left|\int_{0}^{r}\left(\left(\phi_{j(i)}^{(i)}\right)^{\prime}(t)-\left(\phi_{j(i)}^{\left(i_{0}\right)}\right)^{\prime}(t)\right) \mathrm{d} t\right| \\
& =\int_{0}^{r}\left|\left(\phi_{j(i)}^{(i)}\right)^{\prime}(t)-\left(\phi_{j(i)}^{\left(i_{0}\right)}\right)^{\prime}(t)\right| \mathrm{d} t>0
\end{aligned}
$$

because $\left(\phi_{j(i)}^{(i)}\right)^{\prime}(t)-\left(\phi_{j(i)}^{\left(i_{0}\right)}\right)^{\prime}(t)$ may not change its sign due to continuity.

On the basis of these relations, we shall prove that

$$
\forall r \in[0, \bar{r}): \phi(r)=\phi^{\left(i_{0}\right)}(r)
$$

Let us suppose that one can find $\left\{r_{n}\right\}_{n \in \mathbb{N}}, r_{n} \rightarrow 0_{+}$, and $\left\{i_{n}\right\}_{n \in \mathbb{N}}, i_{0} \neq i_{n} \in \mathcal{I}$, such that $\phi\left(r_{n}\right)=\phi^{\left(i_{n}\right)}\left(r_{n}\right)$. Since $\mathcal{I}$ is finite by definition, we may suppose that $r_{n}$ are chosen so that $i_{n}=i_{1}$ for any $n$. From here and (13),

$$
\phi^{\prime}(0)=\lim _{n \rightarrow \infty} \frac{\phi\left(r_{n}\right)-\phi(0)}{r_{n}}=\lim _{n \rightarrow \infty} \frac{\phi^{\left(i_{1}\right)}\left(r_{n}\right)-\phi^{\left(i_{1}\right)}(0)}{r_{n}}=\left(\phi^{\left(i_{1}\right)}\right)^{\prime}(0),
$$




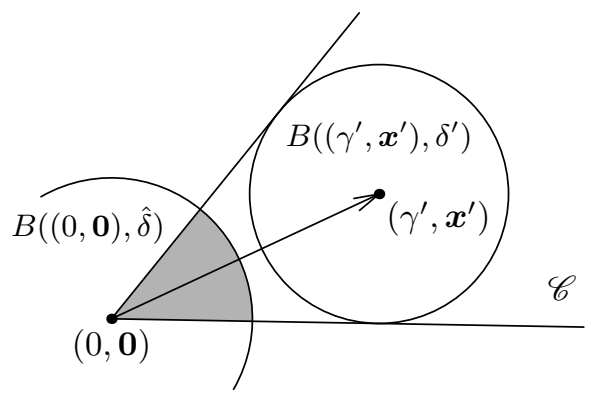

Figure 1: Intersection $\mathscr{C} \cap B((0, \mathbf{0}), \hat{\delta})$.

which contradicts (14). Hence, there exists $\tilde{r}>0$ such that

$$
\forall r \in[0, \tilde{r}): \quad \phi(r)=\phi^{\left(i_{0}\right)}(r) .
$$

From continuity of $\phi$ and $\phi^{(i)}$ and (15), it is readily seen that we can take $\tilde{r}=\bar{r}$, that is, (16) holds. By definition of $\phi$ and $\phi^{\left(i_{0}\right)}$, this means that

$$
\forall r \in[0, \bar{r}) \forall(u, \boldsymbol{v}) \in B\left(\left(\gamma^{\prime}, \boldsymbol{x}^{\prime}\right), \delta^{\prime}\right): \boldsymbol{H}(\bar{\gamma}+r u, \overline{\boldsymbol{x}}+r \boldsymbol{v})=\boldsymbol{H}^{\left(i_{0}\right)}(\bar{\gamma}+r u, \overline{\boldsymbol{x}}+r \boldsymbol{v}) .
$$

Let us introduce a cone $\mathscr{C}$ and a number $\hat{\delta}$ by

$$
\begin{aligned}
\mathscr{C} & :=\bigcup_{r \geq 0} r B\left(\left(\gamma^{\prime}, \boldsymbol{x}^{\prime}\right), \delta^{\prime}\right) \\
\hat{\delta} & :=\bar{r} \min \left\{\|(u, \boldsymbol{v})\| ;(u, \boldsymbol{v}) \in B\left(\left(\gamma^{\prime}, \boldsymbol{x}^{\prime}\right), \delta^{\prime}\right)\right\} .
\end{aligned}
$$

Obviously, $\hat{\delta}$ is positive (by (12), for example) and from (17), it follows that

$$
\forall\left(u^{\prime}, \boldsymbol{v}^{\prime}\right) \in \mathscr{C} \cap B((0, \mathbf{0}), \hat{\delta}): \boldsymbol{H}\left(\bar{\gamma}+u^{\prime}, \overline{\boldsymbol{x}}+\boldsymbol{v}^{\prime}\right)=\boldsymbol{H}^{\left(i_{0}\right)}\left(\bar{\gamma}+u^{\prime}, \overline{\boldsymbol{x}}+\boldsymbol{v}^{\prime}\right)
$$

(see Figure 1). Having this result at hand, it is now easy to verify (9).

Indeed, (8) furnishes us with $\delta>0$ such that

$$
\begin{aligned}
& \forall s \in(\bar{s}, \bar{s}+\delta): \frac{\boldsymbol{c}(s)-\boldsymbol{c}(\bar{s})}{s-\bar{s}} \in B\left(\left(\gamma^{\prime}, \boldsymbol{x}^{\prime}\right), \delta^{\prime}\right), \\
& \forall s \in[\bar{s}, \bar{s}+\delta): \boldsymbol{c}(s)-\boldsymbol{c}(\bar{s}) \in(s-\bar{s}) B\left(\left(\gamma^{\prime}, \boldsymbol{x}^{\prime}\right), \delta^{\prime}\right) \subset \mathscr{C} .
\end{aligned}
$$

Taking into account continuity of $\boldsymbol{c}$ and reducing $\delta$ if necessary, we also have:

$$
\forall s \in[\bar{s}, \bar{s}+\delta): \boldsymbol{c}(s)-\boldsymbol{c}(\bar{s}) \in B((0, \mathbf{0}), \hat{\delta}) .
$$

Thus, invoking $(4)(\mathrm{j})$ and $(18)$, we arrive at (9):

$$
\begin{aligned}
\forall s \in[\bar{s}, \bar{s}+\delta): \boldsymbol{H}^{\left(i_{0}\right)}(\boldsymbol{c}(s)) & =\boldsymbol{H}^{\left(i_{0}\right)}(\boldsymbol{c}(\bar{s})+\boldsymbol{c}(s)-\boldsymbol{c}(\bar{s}))=\boldsymbol{H}^{\left(i_{0}\right)}((\bar{\gamma}, \overline{\boldsymbol{x}})+\boldsymbol{c}(s)-\boldsymbol{c}(\bar{s})) \\
& =\boldsymbol{H}((\bar{\gamma}, \overline{\boldsymbol{x}})+\boldsymbol{c}(s)-\boldsymbol{c}(\bar{s}))=\boldsymbol{H}(\boldsymbol{c}(s))
\end{aligned}
$$


and (4) is proved.

Finally, for any curve $\tilde{\boldsymbol{c}}$ satisfying (5), the same arguments give

$$
\boldsymbol{H}^{\left(i_{0}\right)}(\tilde{\boldsymbol{c}}(s))=\boldsymbol{H}(\tilde{\boldsymbol{c}}(s))=\mathbf{0} \text { for } s \geq \bar{s}, s \text { close to } \bar{s},
$$

and uniqueness of the implicit function determined by the equation $\boldsymbol{H}^{\left(i_{0}\right)}(\gamma, \boldsymbol{x})=\mathbf{0}$ in a vicinity of $(\bar{\gamma}, \overline{\boldsymbol{x}})$ completes the claim.

The following examples of scalar functions of two variables show what can happen when the assumptions (6)(ii) or (iii) of the previous theorem are not fulfilled.

Example 1 (the null direction of the derivative of $\boldsymbol{H}$ is in the kernel of the gradients of more than one selection function). Let $G$ and $H$ be defined by

$$
\begin{aligned}
& G(\gamma, x):= \begin{cases}G^{(1)}(\gamma, x):=-x+\gamma & \text { if } \gamma \leq 0, x \leq 0 ; \\
G^{(2)}(\gamma, x):=-x+\gamma^{2} & \text { if } \gamma \geq 0, x \leq 0 ; \\
G^{(3)}(\gamma, x):=x+\gamma^{2} & \text { if } \gamma \geq 0, x \geq 0 ; \\
G^{(4)}(\gamma, x):=x+\gamma & \text { if } \gamma \leq 0, x \geq 0 ;\end{cases} \\
& H(\gamma, x):= \begin{cases}H^{(1)}(\gamma, x):=x+\gamma^{2} & \text { if } \gamma \leq 0, x \leq 0 ; \\
H^{(2)}(\gamma, x):=x & \text { if } \gamma \geq 0, x \leq 0 ; \\
H^{(3)}(\gamma, x):=-x & \text { if } \gamma \geq 0, x \geq 0 ; \\
H^{(4)}(\gamma, x):=-x+\gamma^{2} & \text { if } \gamma \leq 0, x \geq 0\end{cases}
\end{aligned}
$$

and $(\bar{\gamma}, \bar{x}):=(0,0)$. Then

$$
\begin{aligned}
G^{\prime}((\bar{\gamma}, \bar{x}) ;(1,0)) & =0=\nabla^{\top} G^{(2)}(\bar{\gamma}, \bar{x})(1,0)=\nabla^{\top} G^{(3)}(\bar{\gamma}, \bar{x})(1,0), \\
H^{\prime}((\bar{\gamma}, \bar{x}) ;(1,0)) & =0=\nabla^{\top} H^{(2)}(\bar{\gamma}, \bar{x})(1,0)=\nabla^{\top} H^{(3)}(\bar{\gamma}, \bar{x})(1,0), \\
H^{\prime}((\bar{\gamma}, \bar{x}) ;(-1,0)) & =0=\nabla^{\top} H^{(1)}(\bar{\gamma}, \bar{x})(-1,0)=\nabla^{\top} H^{(4)}(\bar{\gamma}, \bar{x})(-1,0) .
\end{aligned}
$$

However, no solution branch of $G=0$ emanates from $(\bar{\gamma}, \bar{x})$ in the direction $(1,0)$, one solution branch of $H=0$ emanates from $(\bar{\gamma}, \bar{x})$ in the direction $(1,0)$ and two solution branches of $H=0$ emanate from $(\bar{\gamma}, \bar{x})$ in the direction $(-1,0)$ (see Figures 2(a) and (b)). From here, one can see that neither uniqueness nor existence of solution branches can be either guaranteed or excluded in the null direction of the derivative on the basis of the first-order analysis only.

Example 2 (singularity of the partial gradient with respect to $\boldsymbol{x}$ ). Let $H: \mathbb{R}^{2} \rightarrow \mathbb{R}$ be a smooth function defined by $H(\gamma, x):=x\left(x^{2}-\gamma\right)$ (that is, with the only selection function $\left.H^{(1)}=H\right)$ and $(\bar{\gamma}, \bar{x}):=(0,0)$. Then $\nabla^{\top} H(\bar{\gamma}, \bar{x})=(0,0)$ and $H^{\prime}\left((\bar{\gamma}, \bar{x}) ;\left(\gamma^{\prime}, x^{\prime}\right)\right)=0$ for any $\left(\gamma^{\prime}, x^{\prime}\right) \in \mathbb{R}^{2}$. On the other hand, $H^{-1}(0)$ is formed only by two curves, which intersect at $(\bar{\gamma}, \bar{x})$ (see Figure 2(c)).

Let us note that this example is a case of a (smooth) bifurcation (for its definition, see, for instance, [4, Section 24]). In general, if (6)(iii) does not hold, a bifurcation can occur in the solution set of $\boldsymbol{H}^{\left(i_{0}\right)}=\mathbf{0}$, whose subset forms a part of the solution set of $\boldsymbol{H}=\mathbf{0}$. 


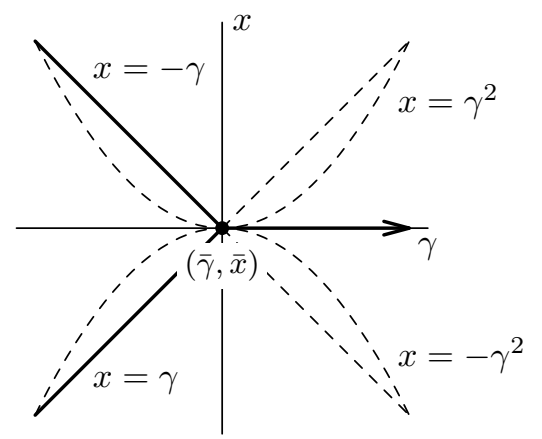

(a) Example 1: $G=0$.

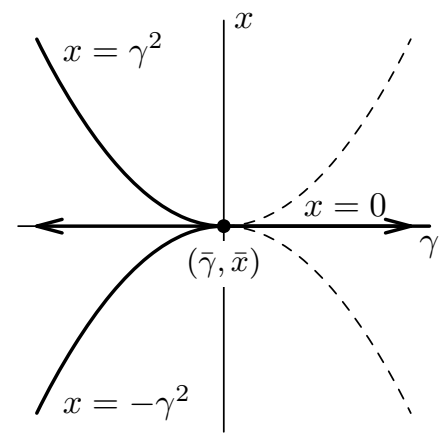

(b) Example 1: $H=0$.

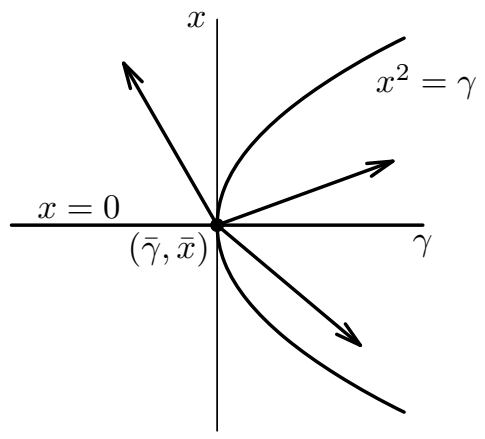

(c) Example 2: $H=0$.

Figure 2: Violation of the assumptions in (6): (a), (b) a null direction in the kernel of the gradients of two selection functions; (c) singularity of the partial gradient with respect to $x$. The arrows represent null directions of derivatives.

Remark 2. Apparently, solution curves of $\boldsymbol{H}(\gamma, \boldsymbol{x})=\mathbf{0}$ are not necessarily always parametrisable by $\gamma$ (this will not be considered even in the next subsection). Nevertheless, it is readily seen that one can interchange the role of $\gamma$ and $\boldsymbol{x}$ with the role of $x_{j}$ and the $N$ tuple $\left(\gamma, x_{1}, \ldots, x_{j-1}, x_{j+1}, \ldots x_{N}\right)$ for any $j=1, \ldots, N$ in the previous analysis and all the results can be generalised in this way. In particular, the assumption (6)(iii) in Theorem 2 can be replaced by the following weaker assumption:

(iii) $\boldsymbol{\nabla} \boldsymbol{H}^{\left(i_{0}\right)}(\bar{\gamma}, \overline{\boldsymbol{x}})$ has $N$ linearly independent columns.

\subsection{Numerical Continuation}

Having extended the analysis of continuation of solution curves in the directions solving the first-order system from [4], we are prepared to describe a method for numerical tracing of $P C^{1}$ solution curves of $(\mathscr{P})$.

We shall not make an explicit difference between the state variable $\boldsymbol{x}$ and the parameter $\gamma$ and we shall consider a uniform formulation of $(\mathscr{P})$, namely,

$$
\boldsymbol{H}(\boldsymbol{y})=\mathbf{0}
$$

with $\boldsymbol{y}=(\gamma, \boldsymbol{x})$. Nevertheless, to avoid bad scaling when calculating tangents, for example, we shall use the following weighted scalar product and norm:

$$
(\boldsymbol{y}, \tilde{\boldsymbol{y}})_{w}:=y_{\gamma} \tilde{y}_{\gamma}+\kappa \boldsymbol{y}_{x}^{\top} \tilde{\boldsymbol{y}}_{x}, \quad\|\boldsymbol{y}\|_{w}:=\sqrt{(\boldsymbol{y}, \boldsymbol{y})_{w}}, \quad \boldsymbol{y}=\left(y_{\gamma}, \boldsymbol{y}_{x}\right), \tilde{\boldsymbol{y}}=\left(\tilde{y}_{\gamma}, \tilde{\boldsymbol{y}}_{x}\right),
$$

as proposed in [16, pp. 86 and 87]. Here, $\kappa$ should be chosen so that $\kappa \boldsymbol{y}_{x}^{\top} \tilde{\boldsymbol{y}}_{x}$ is proportional to the scalar product of the corresponding spatial variables, usually in $L^{2}$. One can take, for instance, $\kappa=h^{d}$, where $h$ is the mesh size and $d$ stands for the dimension of the underlying problem. Alternatively, $\kappa$ can be chosen as $1 / N$ for simplicity, where $N$ is the size of $\boldsymbol{y}_{x}$. 
The principal idea of the present continuation strategy is the same as the one proposed in $[14,12]$, namely, to continue smooth pieces of solution curves by a predictor-corrector method and to join the smooth pieces continuously.

The employed predictor-corrector method is a slight modification of the inexact MoorePenrose continuation implemented in MATCONT [8], which can be viewed as an approximation of a continuation routine making use of the Moore-Penrose pseudo-inverse described in [4]. It computes a sequence of points $\left\{\boldsymbol{y}_{k}\right\}$ lying approximately on a solution curve and a sequence of the corresponding unit tangent vectors $\left\{\boldsymbol{t}_{k}\right\}$. The method consists of two different steps: A predictor step generates an initial approximation of a new point in the direction of the tangent corresponding to the current point. Corrector steps, which are iterative steps of Newton's type, bring the predicted point back to the curve. Since points of non-differentiability of $\boldsymbol{H}$ may be encountered in this procedure, the adaptation of the Newton steps for piecewise-smooth functions [9, 7.2.14 Algorithm] is used and gradients of active selection functions of $\boldsymbol{H}$ are taken, in general. The following algorithm sketches our implementation.

Algorithm 1 (Piecewise-smooth inexact Moore-Penrose predictor-corrector).

Input data: $\varepsilon, \varepsilon^{\prime}>0, c_{\min } \leq 1, h_{\max } \geq h \geq h_{\min }>0, h_{\mathrm{inc}}>1>h_{\mathrm{dec}}>0, j_{\max } \geq j_{\mathrm{thr}}>0$ and $\boldsymbol{y}_{0}, \boldsymbol{t}_{0} \in \mathbb{R}^{N+1}$ satisfying:

$$
\left\|\boldsymbol{H}\left(\boldsymbol{y}_{0}\right)\right\| \leq \varepsilon, \quad \boldsymbol{H}^{\prime}\left(\boldsymbol{y}_{0} ; \boldsymbol{t}_{0}\right)=\mathbf{0}, \quad\left\|\boldsymbol{t}_{0}\right\|_{w}=1
$$

Step 1: Set $k:=0$.

Step 2: Set $n_{\mathrm{dec}}:=0$.

Step 3 (predictor step): Set $j:=0$ and

$$
\boldsymbol{Y}_{0}:=\boldsymbol{y}_{k}+h \boldsymbol{t}_{k}, \quad \boldsymbol{T}_{0}:=\boldsymbol{t}_{k}
$$

Step 4 (corrector step): Select an index $i_{j}$ from $\mathcal{I}_{\boldsymbol{H}}\left(\boldsymbol{Y}_{j}\right)$, find $\boldsymbol{Y}_{j+1}$ and $\tilde{\boldsymbol{T}}$ such that

$$
\begin{array}{rlrl}
\boldsymbol{\nabla} \boldsymbol{H}^{\left(i_{j}\right)}\left(\boldsymbol{Y}_{j}\right)\left(\boldsymbol{Y}_{j+1}-\boldsymbol{Y}_{j}\right) & =-\boldsymbol{H}\left(\boldsymbol{Y}_{j}\right), & \boldsymbol{\nabla} \boldsymbol{H}^{\left(i_{j}\right)}\left(\boldsymbol{Y}_{j}\right) \tilde{\boldsymbol{T}} & =\mathbf{0} \\
\boldsymbol{T}_{j}^{\top}\left(\boldsymbol{Y}_{j+1}-\boldsymbol{Y}_{j}\right) & =0, & \left(\tilde{\boldsymbol{T}}, \boldsymbol{T}_{j}\right)_{w}=1
\end{array}
$$

and set

$$
T_{j+1}:=\tilde{T} /\|\tilde{T}\|_{w}
$$

Step 5: If $\left\|\boldsymbol{H}\left(\boldsymbol{Y}_{j+1}\right)\right\| \leq \varepsilon$ and $\left\|\boldsymbol{Y}_{j+1}-\boldsymbol{Y}_{j}\right\|_{w} \leq \varepsilon^{\prime}$, go to Step 8 .

Step 6: If $j<j_{\max }$, set $j:=j+1$ and go to Step 4 .

Step 7: If $h>h_{\text {min }}$, set $h:=\max \left\{h_{\mathrm{dec}} h, h_{\min }\right\}, n_{\mathrm{dec}}:=n_{\mathrm{dec}}+1$ and go to Step 3 . Otherwise, break; predictor-corrector has failed. 
Step 8: If $\boldsymbol{T}_{j+1}^{\top} \boldsymbol{t}_{k}<c_{\mathrm{min}}$, go to Step 7. Otherwise, set

$$
\begin{gathered}
\boldsymbol{y}_{k+1}:=\boldsymbol{Y}_{j+1}, \quad \boldsymbol{t}_{k+1}:=\boldsymbol{T}_{j+1}, \\
\text { if } j<j_{\text {thr }} \text { and } n_{\mathrm{dec}}=0 \text {, then } h:=\min \left\{h_{\mathrm{inc}} h, h_{\max }\right\} .
\end{gathered}
$$

Set $k:=k+1$ and go to Step 2 .

Here, $\varepsilon$ and $\varepsilon^{\prime}$ are convergence tolerances and $c_{\text {min }}$ serves for controlling changes of direction between tangent vectors at two consecutive points. Further, $h_{\max }$ and $h_{\min }$ are the maximal and the minimal step lengths used in the predictor step, respectively, and $h_{\text {inc }}$ and $h_{\text {dec }}$ are scale factors for adjusting the step length $h$. It is shortened in the case of non-convergence of the corrector or too large deviation between the newly computed tangent and the previous one. On the other hand, it is elongated for the next predictor step if the last computed couple is accepted, the number of corrector steps does not reach $j_{\text {thr }}$ and the number $n_{\text {dec }}$ of step length reductions of $h$ for the current value of $k$ is zero. Finally, $j_{\max }$ stands for the maximal number of corrector steps allowed.

We have chosen the inexact Moore-Penrose predictor-corrector for our continuation technique because it is an efficient path-following method that can traverse smooth folds with respect to the parameter. From our experience, it may pass also over points of non-differentiability if the test on the tangent direction is satisfied. However, we have encountered points of non-differentiability where our predictor-corrector failed to converge and its improvement was needed to overcome this difficulty.

Let $\overline{\boldsymbol{y}}$ be a point of non-differentiability on the solution curve that we want to pass over. The analysis in the previous subsection suggests to compute $\overline{\boldsymbol{y}}$, solve $\boldsymbol{H}^{\prime}(\overline{\boldsymbol{y}} ; \boldsymbol{t})=\mathbf{0}$ for a new tangent direction $\boldsymbol{t}$ and restart the predictor-corrector with $\overline{\boldsymbol{y}}$ and $\boldsymbol{t}$. However, this approach requires exact expressions for sub-domains where $\boldsymbol{H}$ coincides with its individual selection functions. This may not be always easy to obtain.

To avoid this requirement, we shall present another approach that consists in restarting the predictor-corrector with an approximation of the couple $(\overline{\boldsymbol{y}}, \boldsymbol{t})$. We shall explain it in the most probable case when only two selection functions of $\boldsymbol{H}$ are active at $\overline{\boldsymbol{y}}, \boldsymbol{H}^{(1)}$ and $\boldsymbol{H}^{(2)}$, and the adjacent smooth pieces of the solution curve $\boldsymbol{c}$ solve $\boldsymbol{H}^{(1)}=\mathbf{0}$ and $\boldsymbol{H}^{(2)}=\mathbf{0}$, respectively. Moreover, we shall restrict ourselves to the case when the one-sided tangent directions to the solution curve, say $\boldsymbol{t}^{(1)}$ and $\boldsymbol{t}^{(2)}$, do not lie on a line (otherwise, we would have no problem with the tangent predictor) and

$$
\boldsymbol{\nabla} \boldsymbol{H}^{(1)}(\overline{\boldsymbol{y}}) \text { and } \boldsymbol{\nabla} \boldsymbol{H}^{(2)}(\overline{\boldsymbol{y}}) \text { have both the maximal rank } N
$$

(compare also to $(6)(\mathrm{ii})$ and $\left.\left(6^{\prime}\right)(\mathrm{iii})\right)$. For the sake of simplicity of our exposition, we shall also assume that the set $\left\{\boldsymbol{y} \in \mathbb{R}^{N+1} ; \boldsymbol{H}(\boldsymbol{y})=\boldsymbol{H}^{(1)}(\boldsymbol{y})=\boldsymbol{H}^{(2)}(\boldsymbol{y})\right\}$ forms an $N$-dimensional $C^{1}$-manifold in a neighbourhood of $\overline{\boldsymbol{y}}$ (this is satisfied, for example, when there exists a function $G: \mathbb{R}^{N+1} \rightarrow \mathbb{R}$ of the class $C^{1}$ such that $G(\boldsymbol{y})=0$ iff $\boldsymbol{H}^{(1)}(\boldsymbol{y})=\boldsymbol{H}^{(2)}(\boldsymbol{y})$ in a vicinity of $\overline{\boldsymbol{y}}$ and $\nabla G(\overline{\boldsymbol{y}}) \neq \mathbf{0})$. The overall situation is depicted in Figure 3(a).

Now, let an approximation of the part $\left\{\boldsymbol{y} \in \mathbb{R}^{N+1} ; \boldsymbol{H}(\boldsymbol{y})=\boldsymbol{H}^{(1)}(\boldsymbol{y})=\mathbf{0}\right\}$ of the solution curve be known from the predictor-corrector run through till $h=h_{\min }$ and let 


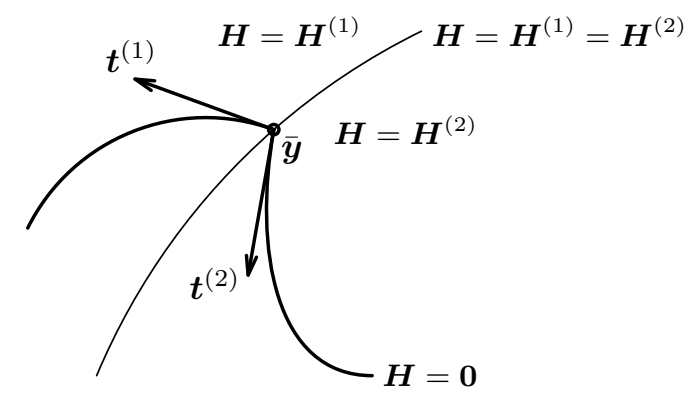

(a)

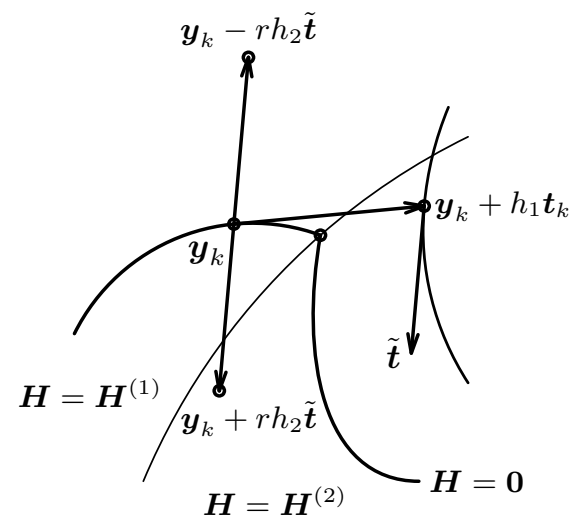

(b)

Figure 3: Transition between smooth pieces of a solution curve.

$\left(\boldsymbol{y}_{k}, \boldsymbol{t}_{k}\right)$ denote the last computed couple. As suggested before, we seek approximations of $\overline{\boldsymbol{y}}$ and $\boldsymbol{t}^{(2)}$ to pass to the part $\left\{\boldsymbol{y} \in \mathbb{R}^{N+1} ; \boldsymbol{H}(\boldsymbol{y})=\boldsymbol{H}^{(2)}(\boldsymbol{y})=\mathbf{0}\right\}$.

Firstly, let us observe that when we choose the minimal step length $h_{\text {min }}$ sufficiently small, the predictor-corrector follows the part of the solution curve corresponding to $\boldsymbol{H}^{(1)}(\boldsymbol{y})=\mathbf{0}$ to the very closeness of $\overline{\boldsymbol{y}}$ as it generates points from the sub-domain $\left\{\boldsymbol{y} \in \mathbb{R}^{N+1} ; \boldsymbol{H}(\boldsymbol{y})=\boldsymbol{H}^{(1)}(\boldsymbol{y})\right\}$ of smooth behaviour. This justifies $\boldsymbol{y}_{k}$ to be a good approximation of $\overline{\boldsymbol{y}}$.

Secondly, to compute an approximation of $\boldsymbol{t}^{(2)}$, we shall make use of the equality

$$
\boldsymbol{H}^{\prime}\left(\overline{\boldsymbol{y}} ; \boldsymbol{t}^{(2)}\right)=\boldsymbol{\nabla} \boldsymbol{H}^{(2)}(\overline{\boldsymbol{y}}) \boldsymbol{t}^{(2)}=\mathbf{0}
$$

(see Figure 3(a)). Figure 3(b) shows that taking $h_{1}$ sufficiently large in comparison with the value of $h_{\min }$ (but not too large to remain in the neighbourhood of $\overline{\boldsymbol{y}}$ where Figure 3(a) is relevant), the point $\boldsymbol{y}_{k}+h_{1} \boldsymbol{t}_{k}$ belongs to the interior of the sub-domain $\left\{\boldsymbol{y} \in \mathbb{R}^{N+1} ; \boldsymbol{H}(\boldsymbol{y})=\right.$ $\left.\boldsymbol{H}^{(2)}(\boldsymbol{y})\right\}$. Therefore, $\boldsymbol{H}$ is Fréchet-differentiable there and

$$
\boldsymbol{\nabla} \boldsymbol{H}\left(\boldsymbol{y}_{k}+h_{1} \boldsymbol{t}_{k}\right)=\boldsymbol{\nabla} \boldsymbol{H}^{(2)}\left(\boldsymbol{y}_{k}+h_{1} \boldsymbol{t}_{k}\right) \approx \boldsymbol{\nabla} \boldsymbol{H}^{(2)}(\overline{\boldsymbol{y}})
$$

if $\boldsymbol{y}_{k}+h_{1} \boldsymbol{t}_{k}$ remains sufficiently close to $\overline{\boldsymbol{y}}$. According to (19), $\boldsymbol{\nabla} \boldsymbol{H}\left(\boldsymbol{y}_{k}+h_{1} \boldsymbol{t}_{k}\right)$ has still the rank $N$ and $\operatorname{span}\left\{\boldsymbol{t}^{(2)}\right\}$ can be approximated by $\operatorname{span}\{\tilde{\boldsymbol{t}}\}$ for $\tilde{\boldsymbol{t}}$ solving

$$
\boldsymbol{\nabla} \boldsymbol{H}\left(\boldsymbol{y}_{k}+h_{1} \boldsymbol{t}_{k}\right) \tilde{\boldsymbol{t}}=\mathbf{0}, \quad\|\tilde{\boldsymbol{t}}\|_{w}=1 .
$$

Finally, we have to determine an appropriate direction of $\tilde{\boldsymbol{t}}$. Figure 3(b) illustrates that there exists $r \in\{ \pm 1\}$ such that $\boldsymbol{y}_{k}-r h_{2} \tilde{\boldsymbol{t}}$ remains in $\left\{\boldsymbol{y} \in \mathbb{R}^{N+1} ; \boldsymbol{H}(\boldsymbol{y})=\boldsymbol{H}^{(1)}(\boldsymbol{y})\right\}$ for any $h_{2}$ positive (still restricting ourselves to the values of $h_{2}$ such that $\boldsymbol{y}_{k} \pm h_{2} \tilde{\boldsymbol{t}}$ is in the neighbourhood of $\overline{\boldsymbol{y}}$ ). This can be recognised by the fact that $\frac{\left|\boldsymbol{t}_{-}^{\top} \tilde{\boldsymbol{t}}\right|}{\left\|\boldsymbol{t}_{-}\right\| \tilde{\boldsymbol{t}} \|}$ is observably smaller than 1 for $\boldsymbol{t}_{-}$with $\boldsymbol{\nabla} \boldsymbol{H}\left(\boldsymbol{y}_{k}-r h_{2} \tilde{\boldsymbol{t}}\right) \boldsymbol{t}_{-}=\mathbf{0}$. 
On the other hand, $\boldsymbol{y}_{k}+r h_{2} \tilde{\boldsymbol{t}}$ appears in $\left\{\boldsymbol{y} \in \mathbb{R}^{N+1} ; \boldsymbol{H}(\boldsymbol{y})=\boldsymbol{H}^{(2)}(\boldsymbol{y})\right\}$ for $h_{2}$ larger than some positive threshold. For such values, $\frac{\left|\boldsymbol{t}_{\boldsymbol{t}}^{\top} \tilde{\boldsymbol{t}}\right|}{\left\|\boldsymbol{t}_{+}\right\|\|\tilde{\boldsymbol{t}}\|}$ is close to 1 for $\boldsymbol{t}_{+}$from the kernel of $\boldsymbol{\nabla} \boldsymbol{H}\left(\boldsymbol{y}_{k}+r h_{2} \tilde{\boldsymbol{t}}\right)$.

This suggests the following procedure for selecting the desired direction of $\tilde{\boldsymbol{t}}$ : Increase the values of $h_{2}$ successively and when you arrive at $h_{2}$ and $r \in\{ \pm 1\}$ such that

$$
\frac{\left|\boldsymbol{t}^{\top} \tilde{\boldsymbol{t}}\right|}{\|\boldsymbol{t}\|\|\tilde{\boldsymbol{t}}\|} \approx 1, \quad \nabla \boldsymbol{H}\left(\boldsymbol{y}_{k}+r h_{2} \tilde{\boldsymbol{t}}\right) \boldsymbol{t}=\mathbf{0}, \quad\|\boldsymbol{t}\|_{w}=1,
$$

take $r \tilde{\boldsymbol{t}}$ as an approximation of $\boldsymbol{t}^{(2)}$.

The overall algorithm for finding a new tangent direction can be sketched as follows.

Algorithm 2 (Simple tangent switch).

Input data: $h_{1}, h_{2}>0, c_{\min } \leq 1$ and $\boldsymbol{y}, \boldsymbol{t} \in \mathbb{R}^{N+1}$ with $\|\boldsymbol{t}\|_{w}=1$.

Step 1: Compute $\tilde{\boldsymbol{t}}$ such that

$$
\boldsymbol{\nabla} \boldsymbol{H}\left(\boldsymbol{y}+h_{1} \boldsymbol{t}\right) \tilde{\boldsymbol{t}}=\mathbf{0}, \quad\|\tilde{\boldsymbol{t}}\|_{w}=1 .
$$

Step 2: Find $\boldsymbol{t}_{+}$and $\boldsymbol{t}_{-}$such that

$$
\boldsymbol{\nabla} \boldsymbol{H}\left(\boldsymbol{y} \pm h_{2} \tilde{\boldsymbol{t}}\right) \boldsymbol{t}_{ \pm}=\mathbf{0}, \quad\left\|\boldsymbol{t}_{ \pm}\right\|_{w}=1 .
$$

Step 3: If $\frac{\left|\boldsymbol{t}_{\boldsymbol{t}}^{\top} \tilde{\mid}\right|}{\left\|\boldsymbol{t}_{+}\right\|\|\tilde{\boldsymbol{t}}\|} \geq c_{\min }$, then set $\boldsymbol{t}:=\tilde{\boldsymbol{t}}$.

Otherwise, if $\frac{\left|\boldsymbol{t}_{\boldsymbol{t}}^{\top} \tilde{\boldsymbol{t}}\right|}{\left\|\boldsymbol{t}_{-}\right\|\|\tilde{\boldsymbol{t}}\|} \geq c_{\min }$, set $\boldsymbol{t}:=-\tilde{\boldsymbol{t}}$.

Otherwise, increase $h_{2}$ and go to Step 2 .

Here, the value of $c_{\min }$ should be chosen the same as or close to the one in Algorithm 1, $h_{1}$ should be a bit greater than $h_{\min }$ from Algorithm 1 and the initial value of $h_{2}$ should be chosen similar to $h_{\min }$. See Subsection 3.2 for our precise settings of the algorithms above.

Even though the simple tangent switch is proposed primarily for points with two active selection functions, it is also applicable to points with more active selection functions according to the analysis in the previous subsection (Theorem 2) and as suggested in Figure 4.

To sum up, the whole numerical continuation of $(\mathscr{P})$ is started with the predictorcorrector (Algorithm 1) with a point $\boldsymbol{y}_{0}$ approximating a solution and the corresponding unit tangent $\boldsymbol{t}_{0}$ in the direction of the desired continuation. If the predictor-corrector fails for some value of $k$, the simple tangent switch (Algorithm 2) is run with the last computed couple $\left(\boldsymbol{y}_{k}, \boldsymbol{t}_{k}\right)$. After finding a new tangent direction $\boldsymbol{t}$, one restarts the predictor-corrector with $\left(\boldsymbol{y}_{k}, \boldsymbol{t}\right)$ and continues tracing a solution curve.

A similar restarted predictor-corrector method has already been successfully tested on simple contact problems in $[14,12]$, where computation of a suitable tangent for restart relies on precise expressions for sub-domains of smooth behaviour. The test examples presented at the end of the next section show that the predictor-corrector works well also with the tangent switch proposed here. So, its obvious advantage is that it is easier to implement for general piecewise-smooth problems. 


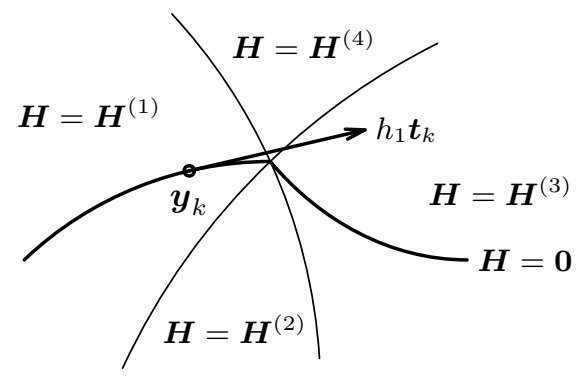

Figure 4: Simple tangent switch in more general situations.

\section{Application to Contact Problems}

The present section focuses on an application of the abstract continuation problem to plane contact problems with friction. We start this exposition with a brief introduction into quasi-static contact problems with Coulomb friction in large deformations. The reader is referred to [7] for a more detailed introduction to large deformations of elastic materials.

Let us consider deformation of an elastic body in a time interval $[0, T]$. The reference configuration of the body is represented by the closure of a bounded domain $\Omega \subset \mathbb{R}^{2}$ with a Lipschitz-continuous boundary $\partial \Omega$. The boundary $\partial \Omega$ is split into three disjoint relatively open subsets $\Gamma_{D}, \Gamma_{N}$ and $\Gamma_{c}$ such that $\partial \Omega=\bar{\Gamma}_{D} \cup \bar{\Gamma}_{N} \cup \bar{\Gamma}_{c}$. By $\boldsymbol{u}$, we denote the displacement of the body and the deformation $\varphi$ of the body is defined by $\boldsymbol{\varphi}=\boldsymbol{i d}+\boldsymbol{u}$, where $\boldsymbol{i d}$ is the identity map. At each time $t \in[0, T]$, the deformed body $\boldsymbol{\varphi}(t, \Omega)$ is subject to body forces with the density $\boldsymbol{f}^{\varphi}$ while surface forces with the density $\boldsymbol{h}^{\varphi}$ act on $\boldsymbol{\varphi}\left(t, \Gamma_{N}\right)$. The displacement $\boldsymbol{U}_{D}$ is imposed on $\Gamma_{D}$ and points from $\Gamma_{c}$ may come into contact with a fixed curved rigid obstacle represented by a closed set $\mathscr{O} \subset \mathbb{R}^{2}$. It is supposed that there exist $g: \mathbb{R}^{2} \rightarrow \mathbb{R}$ of the class $C^{1}$ and a neighbourhood $O$ of the boundary $\partial \mathscr{O}$ such that

$$
\begin{aligned}
& g(\boldsymbol{x})>0, \forall \boldsymbol{x} \in \operatorname{int} \mathscr{O} \cap O, \\
& g(\boldsymbol{x})=0, \forall \boldsymbol{x} \in \partial \mathscr{O}, \\
& g(\boldsymbol{x})<0, \forall \boldsymbol{x} \in \operatorname{ext} \mathscr{O} \cap O,
\end{aligned}
$$

$\nabla g \neq \mathbf{0}$ in $O$ and $\boldsymbol{\varphi}\left(t, \Gamma_{c}\right) \subset O$ for any $t \in[0, T]$. This enables us to extend the unit inward

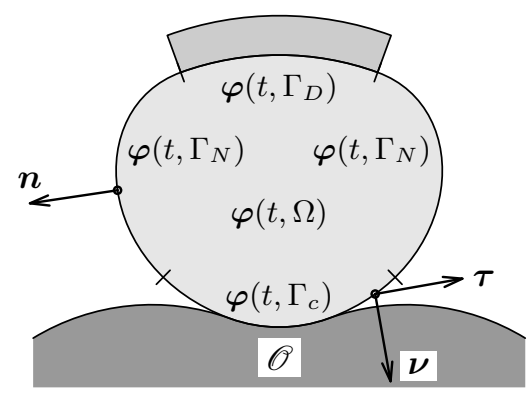

Figure 5: Geometry of the problem. 
normal and tangent vectors to the obstacle to the vicinity $O$ of the obstacle as follows (see Figure 5):

$$
\boldsymbol{\nu}(\boldsymbol{x})=\frac{\nabla g(\boldsymbol{x})}{\|\nabla g(\boldsymbol{x})\|}, \quad \boldsymbol{\tau}(\boldsymbol{x})=\left(-\nu_{2}(\boldsymbol{x}), \nu_{1}(\boldsymbol{x})\right) .
$$

Finally, an initial displacement $\boldsymbol{U}_{0}$ is given at the time $t=0$.

The classical formulation of this evolution problem is the following:

Find $\boldsymbol{u}:[0, T] \times \bar{\Omega} \rightarrow \mathbb{R}^{2}$ such that $\boldsymbol{u}(t,.) \in \mathscr{U}_{\text {ad }}$ for any $t \in[0, T]$ and

$$
\left.\begin{array}{rl}
\operatorname{div} \boldsymbol{\sigma}(t, \boldsymbol{x})+\boldsymbol{f}(t, \boldsymbol{x})=\mathbf{0}, & (t, \boldsymbol{x}) \in(0, T) \times \Omega, \\
\boldsymbol{\sigma}(t, \boldsymbol{x})=\hat{\boldsymbol{\sigma}}(\boldsymbol{x}, \boldsymbol{I}+\boldsymbol{\nabla} \boldsymbol{u}(t, \boldsymbol{x})), & (t, \boldsymbol{x}) \in(0, T) \times \Omega, \\
\boldsymbol{u}(t, \boldsymbol{x})=\boldsymbol{U}_{D}(t, \boldsymbol{x}), & (t, \boldsymbol{x}) \in(0, T) \times \Gamma_{D}, \\
\boldsymbol{\sigma}(t, \boldsymbol{x}) \boldsymbol{n}(\boldsymbol{x})=\boldsymbol{h}(t, \boldsymbol{x}), & (t, \boldsymbol{x}) \in(0, T) \times \Gamma_{N}, \\
g(\boldsymbol{x}+\boldsymbol{u}(t, \boldsymbol{x})) \leq 0, T_{\nu}(t, \boldsymbol{x}) \leq 0, g(\boldsymbol{x}+\boldsymbol{u}(t, \boldsymbol{x})) T_{\nu}(t, \boldsymbol{x})=0, & (t, \boldsymbol{x}) \in(0, T) \times \Gamma_{c}, \\
\left|T_{\tau}(t, \boldsymbol{x})\right| \leq-\mathscr{F}(\boldsymbol{x}) T_{\nu}(t, \boldsymbol{x}), & (t, \boldsymbol{x}) \in(0, T) \times \Gamma_{c}, \\
T_{\tau}(t, \boldsymbol{x})=\mathscr{F}(\boldsymbol{x}) T_{\nu}(t, \boldsymbol{x}) \frac{\dot{u}_{\tau}(t, \boldsymbol{x})}{\left|\dot{u}_{\tau}(t, \boldsymbol{x})\right|} \quad \text { if } \dot{u}_{\tau}(t, \boldsymbol{x}) \neq 0, & (t, \boldsymbol{x}) \in(0, T) \times \Gamma_{c}, \\
\boldsymbol{u}(0, \boldsymbol{x})=\boldsymbol{U}_{0}(\boldsymbol{x}), & \boldsymbol{x} \in \Omega .
\end{array}\right\}
$$

Here, $\boldsymbol{I}$ denotes the identity matrix, $\boldsymbol{\nabla} \boldsymbol{u}$ is the partial gradient of $\boldsymbol{u}$ with respect to $\boldsymbol{x}$ and $\mathscr{U}_{\text {ad }}$ is the set of kinematically admissible displacements:

$\mathscr{U}_{\mathrm{ad}}=\left\{\boldsymbol{v}: \bar{\Omega} \rightarrow \mathbb{R}^{2}\right.$ "smooth enough"; $\boldsymbol{i d}+\boldsymbol{v}$ is injective on $\Omega, \operatorname{det}(\boldsymbol{I}+\boldsymbol{\nabla} \boldsymbol{v})>0$ in $\left.\bar{\Omega}\right\}$.

The first Piola-Kirchhoff stress tensor $\boldsymbol{\sigma}$ is defined from the Cauchy stress tensor $\boldsymbol{\sigma}^{\boldsymbol{\varphi}}$ by

$$
\boldsymbol{\sigma}(t, \boldsymbol{x})=(\operatorname{det}(\boldsymbol{I}+\boldsymbol{\nabla} \boldsymbol{u}(t, \boldsymbol{x}))) \boldsymbol{\sigma}^{\boldsymbol{\varphi}}(t, \boldsymbol{x}+\boldsymbol{u}(t, \boldsymbol{x}))(\boldsymbol{I}+\boldsymbol{\nabla} \boldsymbol{u}(t, \boldsymbol{x}))^{-\top}
$$

and $\hat{\boldsymbol{\sigma}}$ is a given response function for the first Piola-Kirchhoff stress of the elastic material of the body. By $\boldsymbol{n}$, we denote the unit outward normal vector along $\partial \Omega$ and

$$
\begin{aligned}
& \boldsymbol{f}(t, \boldsymbol{x})=\operatorname{det}(\boldsymbol{I}+\boldsymbol{\nabla} \boldsymbol{u}(t, \boldsymbol{x})) \boldsymbol{f}^{\boldsymbol{\varphi}}(t, \boldsymbol{x}+\boldsymbol{u}(t, \boldsymbol{x})), \\
& \boldsymbol{h}(t, \boldsymbol{x})=\operatorname{det}(\boldsymbol{I}+\boldsymbol{\nabla} \boldsymbol{u}(t, \boldsymbol{x}))\left\|(\boldsymbol{I}+\boldsymbol{\nabla} \boldsymbol{u}(t, \boldsymbol{x}))^{-\top} \boldsymbol{n}(\boldsymbol{x})\right\| \boldsymbol{h}^{\varphi}(t, \boldsymbol{x}+\boldsymbol{u}(t, \boldsymbol{x}))
\end{aligned}
$$

are the densities of volume and surface forces related to the reference configuration. Further,

$$
T_{\nu}(t, \boldsymbol{x})=\boldsymbol{T}(t, \boldsymbol{x}) \cdot \boldsymbol{\nu}(\boldsymbol{x}+\boldsymbol{u}(t, \boldsymbol{x})), \quad T_{\tau}(t, \boldsymbol{x})=\boldsymbol{T}(t, \boldsymbol{x}) \cdot \boldsymbol{\tau}(\boldsymbol{x}+\boldsymbol{u}(t, \boldsymbol{x}))
$$

are components of the first Piola-Kirchhoff stress vector $\boldsymbol{T}$ at $(t, \boldsymbol{x})$ in the directions $\boldsymbol{\nu}$ and $\boldsymbol{\tau}, \boldsymbol{T}(t, \boldsymbol{x})=\boldsymbol{\sigma}(t, \boldsymbol{x}) \boldsymbol{n}(\boldsymbol{x})$. The friction coefficient $\mathscr{F}$ is a non-negative function of $\boldsymbol{x}$ and

$$
\dot{u}_{\tau}(t, \boldsymbol{x})=\frac{\partial \boldsymbol{u}}{\partial t}(t, \boldsymbol{x}) \cdot \boldsymbol{\tau}(\boldsymbol{x}+\boldsymbol{u}(t, \boldsymbol{x}))
$$

is the tangent velocity of a point $\boldsymbol{x}$ at a time $t$. 
To interpret the boundary conditions on $\Gamma_{c}$, let us consider $(t, \boldsymbol{x}) \in(0, T) \times \Gamma_{c}$ fixed and assume that $\boldsymbol{\varphi}$ is sufficiently smooth so that $\boldsymbol{\varphi}(t, \Omega)$ is a bounded domain with a Lipschitz-continuous boundary $\partial \boldsymbol{\varphi}(t, \Omega)$ and $\partial \boldsymbol{\varphi}(t, \Omega)=\boldsymbol{\varphi}(t, \partial \Omega)$. If $\boldsymbol{x}^{\boldsymbol{\varphi}}:=\boldsymbol{x}+\boldsymbol{u}(t, \boldsymbol{x})$ denotes the current position of the point $\boldsymbol{x}$ at the time $t$, then the unit outward normal vector $\boldsymbol{n}^{\varphi}$ along $\partial \boldsymbol{\varphi}(t, \Omega)$ can be expressed according to [7, Section 1.7] as

$$
\boldsymbol{n}^{\varphi}\left(\boldsymbol{x}^{\varphi}\right)=\frac{(\boldsymbol{I}+\boldsymbol{\nabla} \boldsymbol{u}(t, \boldsymbol{x}))^{-\top} \boldsymbol{n}(\boldsymbol{x})}{\left\|(\boldsymbol{I}+\boldsymbol{\nabla u}(t, \boldsymbol{x}))^{-\top} \boldsymbol{n}(\boldsymbol{x})\right\|} .
$$

From here and (21), the Cauchy stress vector $\boldsymbol{T}^{\varphi}, \boldsymbol{T}^{\varphi}\left(t, \boldsymbol{x}^{\varphi}\right)=\boldsymbol{\sigma}^{\varphi}\left(t, \boldsymbol{x}^{\varphi}\right) \boldsymbol{n}^{\varphi}\left(\boldsymbol{x}^{\varphi}\right)$, can be written as

$$
\boldsymbol{T}^{\boldsymbol{\varphi}}\left(t, \boldsymbol{x}^{\boldsymbol{\varphi}}\right)=\frac{1}{\operatorname{det}(\boldsymbol{I}+\boldsymbol{\nabla} \boldsymbol{u}(t, \boldsymbol{x}))\left\|(\boldsymbol{I}+\boldsymbol{\nabla} \boldsymbol{u}(t, \boldsymbol{x}))^{-\top} \boldsymbol{n}(\boldsymbol{x})\right\|} \boldsymbol{T}(t, \boldsymbol{x})
$$

and it is readily seen that the contact conditions $(20)_{6-8}$ at $(t, \boldsymbol{x})$ are equivalent to

$$
\begin{aligned}
g\left(\boldsymbol{x}^{\boldsymbol{\varphi}}\right) & \leq 0, \quad T_{\nu}^{\boldsymbol{\varphi}}\left(t, \boldsymbol{x}^{\boldsymbol{\varphi}}\right) \leq 0, \quad g\left(\boldsymbol{x}^{\boldsymbol{\varphi}}\right) T_{\nu}^{\boldsymbol{\varphi}}\left(t, \boldsymbol{x}^{\boldsymbol{\varphi}}\right)=0, \\
\left|T_{\tau}^{\boldsymbol{\varphi}}\left(t, \boldsymbol{x}^{\boldsymbol{\varphi}}\right)\right| & \leq-\mathscr{F}^{\boldsymbol{\varphi}}\left(\boldsymbol{x}^{\boldsymbol{\varphi}}\right) T_{\nu}^{\boldsymbol{\varphi}}\left(t, \boldsymbol{x}^{\boldsymbol{\varphi}}\right), \\
T_{\tau}^{\boldsymbol{\varphi}}\left(t, \boldsymbol{x}^{\boldsymbol{\varphi}}\right) & =\mathscr{F}^{\boldsymbol{\varphi}}\left(\boldsymbol{x}^{\boldsymbol{\varphi}}\right) T_{\nu}^{\boldsymbol{\varphi}}\left(t, \boldsymbol{x}^{\boldsymbol{\varphi}}\right) \frac{v_{\tau}^{\varphi}\left(t, \boldsymbol{x}^{\boldsymbol{\varphi}}\right)}{\left|v_{\tau}^{\boldsymbol{\varphi}}\left(t, \boldsymbol{x}^{\boldsymbol{\varphi}}\right)\right|} \quad \text { if } v_{\tau}^{\boldsymbol{\varphi}}\left(t, \boldsymbol{x}^{\boldsymbol{\varphi}}\right) \neq 0 .
\end{aligned}
$$

Here, $v_{\tau}^{\varphi}\left(t, \boldsymbol{x}^{\boldsymbol{\varphi}}\right)=\dot{u}_{\tau}(t, \boldsymbol{x})$ is the tangent velocity of the point with the current position $\boldsymbol{x}^{\varphi}, \mathscr{F} \boldsymbol{\varphi}\left(\boldsymbol{x}^{\varphi}\right)=\mathscr{F}(\boldsymbol{x})$ and

$$
T_{\nu}^{\varphi}\left(t, \boldsymbol{x}^{\varphi}\right)=\boldsymbol{T}^{\varphi}\left(t, \boldsymbol{x}^{\varphi}\right) \cdot \boldsymbol{\nu}\left(\boldsymbol{x}^{\varphi}\right), \quad T_{\tau}^{\varphi}\left(t, \boldsymbol{x}^{\varphi}\right)=\boldsymbol{T}^{\varphi}\left(t, \boldsymbol{x}^{\varphi}\right) \cdot \boldsymbol{\tau}\left(\boldsymbol{x}^{\varphi}\right) .
$$

In this way, we obtain the unilateral condition and the Coulomb friction law in the part $\boldsymbol{\varphi}\left(t, \Gamma_{c}\right)$ of the boundary $\partial \boldsymbol{\varphi}(t, \Omega)$ of the deformed configuration.

Now, we shall describe briefly discretisation of the problem. Spatial discretisation is done by applying a Lagrange finite-element method to a mixed variational formulation of (20) with Lagrange multipliers enforcing the Dirichlet and contact boundary conditions. In particular, the contact conditions are discretised similarly as in the example of the "almost conformal" discretisation of the displacement in [20, Section 4].

Let $\left\{\boldsymbol{\phi}_{i}\right\}_{1 \leq i \leq 2 n_{\Omega}}$ be a basis of shape functions for displacements and $\left\{\boldsymbol{x}_{i}\right\}_{1 \leq i \leq n_{\Omega}}$ be the set of the corresponding finite-element nodes such that

$$
\boldsymbol{\phi}_{i}\left(\boldsymbol{x}_{j}\right)=\left(\delta_{i, 2 j-1}, \delta_{i, 2 j}\right)
$$

where $\delta$ denotes the Kronecker delta. Let $\left\{\boldsymbol{\xi}_{i}\right\}_{1 \leq i \leq 2 n_{D}}$ be a basis of shape functions on $\bar{\Gamma}_{D}$. By $\left\{\boldsymbol{y}_{i}\right\}_{1 \leq i \leq n_{c}}$, we denote the nodes from $\left\{\boldsymbol{x}_{i}\right\}_{1 \leq i \leq n_{\Omega}}$ lying on $\bar{\Gamma}_{c} \backslash \bar{\Gamma}_{D}$ and by $\left\{\eta_{i}\right\}_{1 \leq i \leq n_{c}}$, the basis of shape functions on $\bar{\Gamma}_{c}$ formed by the restrictions of appropriate component functions of $\phi_{i}$ onto $\bar{\Gamma}_{c}$ such that

$$
\eta_{i}\left(\boldsymbol{y}_{j}\right)=\delta_{i j}
$$


The local and global numeration of the nodes on $\bar{\Gamma}_{c} \backslash \bar{\Gamma}_{D}$ are linked by the mapping $\Theta:\left\{1, \ldots, n_{c}\right\} \rightarrow\left\{1, \ldots, n_{\Omega}\right\}$ such that $\boldsymbol{y}_{i}=\boldsymbol{x}_{\Theta(i)}$. In what follows, we shall assume that the finite-element partitions corresponding to the bases $\left\{\boldsymbol{\phi}_{i}\right\}_{1 \leq i \leq 2 n_{\Omega}}$ and $\left\{\boldsymbol{\xi}_{i}\right\}_{1 \leq i \leq 2 n_{D}}$ are compatible with the decomposition of $\partial \Omega$ into $\bar{\Gamma}_{D}, \bar{\Gamma}_{N}$ and $\bar{\Gamma}_{c}$.

We introduce the following vectors and matrices for $\boldsymbol{w}=\left(w_{i}\right)_{1 \leq i \leq 2 n_{\Omega}}$ and $t \in[0, T]$ :

$$
\begin{aligned}
& \boldsymbol{A}(\boldsymbol{w})=\left(A_{i}(\boldsymbol{w})\right)_{1 \leq i \leq 2 n_{\Omega}}, \quad A_{i}(\boldsymbol{w})=\int_{\Omega} \hat{\boldsymbol{\sigma}}\left(\boldsymbol{x}, \boldsymbol{I}+\sum_{j=1}^{2 n_{\Omega}} w_{j} \boldsymbol{\nabla} \boldsymbol{\phi}_{j}(\boldsymbol{x})\right): \nabla \phi_{i}(\boldsymbol{x}) \mathrm{d} \boldsymbol{x} \\
& \boldsymbol{B}_{D}=\left(B_{D, i j}\right)_{\substack{1 \leq i \leq 2 n_{D} \\
1 \leq j \leq 2 n_{\Omega}}}, \quad B_{D, i j}=\int_{\Gamma_{D}} \boldsymbol{\xi}_{i}(\boldsymbol{x}) \cdot \boldsymbol{\phi}_{j}(\boldsymbol{x}) \mathrm{d} s, \\
& \boldsymbol{B}_{\nu}(\boldsymbol{w})=\left(B_{\nu, i j}(\boldsymbol{w})\right)_{\substack{1 \leq i \leq n_{c} \\
1 \leq j \leq 2 n_{\Omega}}} \quad B_{\nu, i j}(\boldsymbol{w})= \begin{cases}\nu_{1}\left(\boldsymbol{y}_{i}+\left(w_{2 \Theta(i)-1}, w_{2 \Theta(i)}\right)\right) & \text { if } j=2 \Theta(i)-1, \\
\nu_{2}\left(\boldsymbol{y}_{i}+\left(w_{2 \Theta(i)-1}, w_{2 \Theta(i)}\right)\right) & \text { if } j=2 \Theta(i), \\
0 & \text { otherwise, }\end{cases} \\
& \boldsymbol{B}_{\tau}(\boldsymbol{w})=\left(B_{\tau, i j}(\boldsymbol{w})\right)_{\substack{1 \leq i \leq n_{c} \\
1 \leq j \leq 2 n_{\Omega}}} \quad B_{\tau, i j}(\boldsymbol{w})= \begin{cases}\tau_{1}\left(\boldsymbol{y}_{i}+\left(w_{2 \Theta(i)-1}, w_{2 \Theta(i)}\right)\right) & \text { if } j=2 \Theta(i)-1, \\
\tau_{2}\left(\boldsymbol{y}_{i}+\left(w_{2 \Theta(i)-1}, w_{2 \Theta(i)}\right)\right) & \text { if } j=2 \Theta(i), \\
0 & \text { otherwise, }\end{cases} \\
& \boldsymbol{l}(t, \boldsymbol{w})=\left(l_{i}(t, \boldsymbol{w})\right)_{1 \leq i \leq 2 n_{\Omega}}, \\
& l_{i}(t, \boldsymbol{w})=\int_{\Omega}\left(\operatorname{det}\left(\boldsymbol{I}+\sum_{j=1}^{2 n_{\Omega}} w_{j} \boldsymbol{\nabla} \boldsymbol{\phi}_{j}(\boldsymbol{x})\right)\right) \boldsymbol{f}^{\varphi}\left(t, \boldsymbol{x}+\sum_{j=1}^{2 n_{\Omega}} w_{j} \boldsymbol{\phi}_{j}(\boldsymbol{x})\right) \cdot \boldsymbol{\phi}_{i}(\boldsymbol{x}) \mathrm{d} \boldsymbol{x} \\
& +\int_{\Gamma_{N}}\left(\operatorname{det}\left(\boldsymbol{I}+\sum_{j=1}^{2 n_{\Omega}} w_{j} \boldsymbol{\nabla} \boldsymbol{\phi}_{j}(\boldsymbol{x})\right)\right)\left\|\left(\boldsymbol{I}+\sum_{j=1}^{2 n_{\Omega}} w_{j} \boldsymbol{\nabla} \boldsymbol{\phi}_{j}(\boldsymbol{x})\right)^{-\top} \boldsymbol{n}(\boldsymbol{x})\right\| \\
& \cdot \boldsymbol{h}^{\varphi}\left(t, \boldsymbol{x}+\sum_{j=1}^{2 n_{\Omega}} w_{j} \boldsymbol{\phi}_{j}(\boldsymbol{x})\right) \cdot \boldsymbol{\phi}_{i}(\boldsymbol{x}) \mathrm{d} s, \\
& \boldsymbol{u}_{D}(t)=\left(u_{D, i}(t)\right)_{1 \leq i \leq 2 n_{D}}, \quad u_{D, i}(t)=\int_{\Gamma_{D}} \boldsymbol{U}_{D}(t, \boldsymbol{x}) \cdot \boldsymbol{\xi}_{i}(\boldsymbol{x}) \mathrm{d} s, \\
& \boldsymbol{g}(\boldsymbol{w})=\left(g_{i}(\boldsymbol{w})\right)_{1 \leq i \leq n_{c}}, \quad g_{i}(\boldsymbol{w})=g\left(\boldsymbol{y}_{i}+\left(w_{2 \Theta(i)-1}, w_{2 \Theta(i)}\right)\right), \\
& \mathscr{F}=\left(\mathscr{F}_{i}\right)_{1 \leq i \leq n_{c}}, \quad \mathscr{F}_{i}=\mathscr{F}\left(\boldsymbol{y}_{i}\right) .
\end{aligned}
$$

Furthermore, we take $\boldsymbol{u}^{0}=\left(u_{i}^{0}\right)_{1 \leq i \leq 2 n_{\Omega}}$ such that $\sum_{i=1}^{2 n_{\Omega}} u_{i}^{0} \boldsymbol{\phi}_{i}$ approximates $\boldsymbol{U}_{0}$ and we define the sets:

$$
\begin{aligned}
\boldsymbol{\Lambda}_{\nu} & =\mathbb{R}_{-}^{n_{c}} \equiv\left\{\boldsymbol{\mu}_{\nu}=\left(\mu_{\nu, i}\right)_{1 \leq i \leq n_{c}} ; \mu_{\nu, i} \leq 0\right\} \\
\boldsymbol{\Lambda}_{\tau}\left(\mathscr{F} \boldsymbol{\mu}_{\nu}\right) & =\left\{\boldsymbol{\mu}_{\tau}=\left(\mu_{\tau, i}\right)_{1 \leq i \leq n_{c}} ;\left|\mu_{\tau, i}\right| \leq-\mathscr{F}_{i} \mu_{\nu, i}\right\}, \quad \boldsymbol{\mu}_{\nu} \in \boldsymbol{\Lambda}_{\nu} .
\end{aligned}
$$


Then, the spatial semi-discretisation of (20) can be formulated as follows:

Find $\boldsymbol{u}:[0, T] \rightarrow \mathbb{R}^{2 n_{\Omega}}, \boldsymbol{\lambda}_{D}:[0, T] \rightarrow \mathbb{R}^{2 n_{D}}, \boldsymbol{\lambda}_{\nu}, \boldsymbol{\lambda}_{\tau}:[0, T] \rightarrow \mathbb{R}^{n_{c}}$ such that $\boldsymbol{u}(0)=\boldsymbol{u}^{0}$ and for any $t \in(0, T)$, it holds that $\boldsymbol{\lambda}_{\nu}(t) \in \boldsymbol{\Lambda}_{\nu}, \boldsymbol{\lambda}_{\tau}(t) \in \boldsymbol{\Lambda}_{\tau}\left(\mathscr{F} \boldsymbol{\lambda}_{\nu}(t)\right)$ and

$$
\left.\begin{array}{rl}
\boldsymbol{A}(\boldsymbol{u}(t))-\boldsymbol{B}_{D}^{\top} \boldsymbol{\lambda}_{D}(t)-\boldsymbol{B}_{\nu}^{\top}(\boldsymbol{u}(t)) \boldsymbol{\lambda}_{\nu}(t)-\boldsymbol{B}_{\tau}^{\top}(\boldsymbol{u}(t)) \boldsymbol{\lambda}_{\tau}(t) & =\boldsymbol{l}(t, \boldsymbol{u}(t)), \\
\boldsymbol{B}_{D} \boldsymbol{u}(t) & =\boldsymbol{u}_{D}(t), \\
\left(\boldsymbol{\mu}_{\nu}-\boldsymbol{\lambda}_{\nu}(t)\right)^{\top} \boldsymbol{g}(\boldsymbol{u}(t)) & \geq 0, \quad \forall \boldsymbol{\mu}_{\nu} \in \boldsymbol{\Lambda}_{\nu}, \\
\left(\boldsymbol{\mu}_{\tau}-\boldsymbol{\lambda}_{\tau}(t)\right)^{\top} \boldsymbol{B}_{\tau}(\boldsymbol{u}(t)) \dot{\boldsymbol{u}}(t) & \geq 0, \quad \forall \boldsymbol{\mu}_{\tau} \in \boldsymbol{\Lambda}_{\tau}\left(\mathscr{F} \boldsymbol{\lambda}_{\nu}(t)\right),
\end{array}\right\}
$$

where $\boldsymbol{u}$ is the vector of nodal displacements, $\boldsymbol{\lambda}_{D}$ is the Lagrange multiplier corresponding to the Dirichlet condition and $\boldsymbol{\lambda}_{\nu}, \boldsymbol{\lambda}_{\tau}$ are the normal and tangential Lagrange multipliers on the contact zone, respectively.

Approximating $\dot{\boldsymbol{u}}(t)$ by a backward difference and employing the idea from the abstract frame, one obtains the continuation problem:

Find $\gamma \in \mathbb{R}, \boldsymbol{u} \in \mathbb{R}^{2 n_{\Omega}}, \boldsymbol{\lambda}_{D} \in \mathbb{R}^{2 n_{D}}, \boldsymbol{\lambda}_{\nu} \in \boldsymbol{\Lambda}_{\nu}, \boldsymbol{\lambda}_{\tau} \in \boldsymbol{\Lambda}_{\tau}\left(\mathscr{F} \boldsymbol{\lambda}_{\nu}\right)$ such that

$$
\begin{aligned}
\boldsymbol{A}(\boldsymbol{u})-\boldsymbol{B}_{D}^{\top} \boldsymbol{\lambda}_{D}-\boldsymbol{B}_{\nu}^{\top}(\boldsymbol{u}) \boldsymbol{\lambda}_{\nu}-\boldsymbol{B}_{\tau}^{\top}(\boldsymbol{u}) \boldsymbol{\lambda}_{\tau} & =\boldsymbol{L}(\gamma, \boldsymbol{u}), \\
\boldsymbol{B}_{D} \boldsymbol{u} & =\boldsymbol{U}_{D}(\gamma), \\
\left(\boldsymbol{\mu}_{\nu}-\boldsymbol{\lambda}_{\nu}\right)^{\top} \boldsymbol{g}(\boldsymbol{u}) & \geq 0, \quad \forall \boldsymbol{\mu}_{\nu} \in \boldsymbol{\Lambda}_{\nu}, \\
\left(\boldsymbol{\mu}_{\tau}-\boldsymbol{\lambda}_{\tau}\right)^{\top}\left(\boldsymbol{B}_{\tau}(\boldsymbol{u}) \boldsymbol{u}-\boldsymbol{B}_{\tau}(\boldsymbol{u}) \boldsymbol{w}+(1-\gamma) \boldsymbol{V}_{\tau}\right) & \geq 0, \quad \forall \boldsymbol{\mu}_{\tau} \in \boldsymbol{\Lambda}_{\tau}\left(\mathscr{F} \boldsymbol{\lambda}_{\nu}\right),
\end{aligned}
$$

with

$$
\begin{gathered}
\boldsymbol{L}(\gamma, \boldsymbol{u})=\gamma \boldsymbol{l}\left(t_{k+1}, \boldsymbol{u}\right)+(1-\gamma) \boldsymbol{l}\left(t_{k}, \boldsymbol{u}\right), \quad \boldsymbol{U}_{D}(\gamma)=\gamma \boldsymbol{u}_{D}\left(t_{k+1}\right)+(1-\gamma) \boldsymbol{u}_{D}\left(t_{k}\right) \\
\boldsymbol{w}=\boldsymbol{u}^{k}, \quad \boldsymbol{V}_{\tau}=\left(t_{k+1}-t_{k}\right) \frac{\boldsymbol{B}_{\tau}\left(\boldsymbol{u}^{k}\right) \boldsymbol{u}^{k}-\boldsymbol{B}_{\tau}\left(\boldsymbol{u}^{k}\right) \boldsymbol{u}^{k-1}}{t_{k}-t_{k-1}}
\end{gathered}
$$

As in the abstract frame, the benefit of this continuation problem is that any solution from the $k$ th time level of the corresponding fully discretised problem yields always a solution of the continuation problem, which can be taken as a starting point. Moreover, any solution of the continuation problem with $\gamma=1$ yields a solution of the discretised (incremental) problem on the $(k+1)$ st time level.

As $\boldsymbol{L}$ and $\boldsymbol{U}_{D}$, which are parametrised by $\gamma$, represent loadings, $\gamma$ may be viewed as a loading parameter in this problem. Nevertheless, as already emphasised in the abstract frame, $\gamma$ does not play the exact role of time. It serves merely for a transition between two time levels of the fully discretised problem for a fixed time increment!

Due to variational characterisation of a projection onto a closed convex set, this continuation problem is equivalent to

$$
\left.\begin{array}{l}
\text { Find }\left(\gamma, \boldsymbol{u}, \boldsymbol{\lambda}_{D}, \boldsymbol{\lambda}_{\nu}, \boldsymbol{\lambda}_{\tau}\right) \in \mathbb{R}^{1+2\left(n_{\Omega}+n_{D}+n_{c}\right)} \text { such that } \\
\boldsymbol{H}\left(\gamma, \boldsymbol{u}, \boldsymbol{\lambda}_{D}, \boldsymbol{\lambda}_{\nu}, \boldsymbol{\lambda}_{\tau}\right)=\mathbf{0}
\end{array}\right\}
$$


where $\boldsymbol{H}: \mathbb{R}^{1+2\left(n_{\Omega}+n_{D}+n_{c}\right)} \rightarrow \mathbb{R}^{2\left(n_{\Omega}+n_{D}+n_{c}\right)}$ is defined by

$$
\boldsymbol{H}\left(\gamma, \boldsymbol{u}, \boldsymbol{\lambda}_{D}, \boldsymbol{\lambda}_{\nu}, \boldsymbol{\lambda}_{\tau}\right)=\left(\begin{array}{c}
\boldsymbol{A}(\boldsymbol{u})-\boldsymbol{L}(\gamma, \boldsymbol{u})-\boldsymbol{B}_{D}^{\top} \boldsymbol{\lambda}_{D}-\boldsymbol{B}_{\nu}^{\top}(\boldsymbol{u}) \boldsymbol{\lambda}_{\nu}-\boldsymbol{B}_{\tau}^{\top}(\boldsymbol{u}) \boldsymbol{\lambda}_{\tau} \\
\boldsymbol{B}_{D} \boldsymbol{u}-\boldsymbol{U}_{D}(\gamma) \\
\lambda_{\nu, j}-\left(\lambda_{\nu, j}-g_{j}(\boldsymbol{u})\right)_{-}, \quad j=1, \ldots, n_{c} \\
\lambda_{\tau, j}-P_{\left[\mathscr{F}_{j}\left(\lambda_{\nu, j}-g_{j}(\boldsymbol{u})\right)_{-},-\mathscr{F}_{j}\left(\lambda_{\nu, j}-g_{j}(\boldsymbol{u})\right)_{-}\right]}\left(\lambda_{\tau, j}\right. \\
\left.-\left(\boldsymbol{B}_{\tau}(\boldsymbol{u}) \boldsymbol{u}-\boldsymbol{B}_{\tau}(\boldsymbol{u}) \boldsymbol{w}\right)_{j}-(1-\gamma) V_{\tau, j}\right), \quad j=1, \ldots, n_{c}
\end{array}\right)
$$

(see also [2, Section 3]). Here, $(a)_{-}$is the non-positive part of $a$, or equivalently, the projection of $a$ onto the interval $(-\infty, 0]$, and $P_{[a, b]}$ stands for the projection onto an interval $[a, b]$.

Since the functions $(a, b) \mapsto a-(a-b)_{-}$and $(a, b, c) \mapsto a-P_{\left[k c_{-},-k c_{-}\right]}(a-b), k \geq 0$, are both $P C^{1}$ (see Figure 6) and a composition of two $P C^{1}$-functions is again $P C^{1}, \boldsymbol{H}$ is a $P C^{1}$-function under the following assumption:

Assumption 1. Let $\boldsymbol{A}$ and $\boldsymbol{l}(t,),. t \in[0, T]$, be $C^{1}$-functions and $g$ demarcating the rigid obstacle be of the class $C^{2}$.

In this case, the general theory from the previous section can be applied.

Remark 3. In the present discretisation, we treat the Dirichlet condition via a Lagrange multiplier so that it can be simply parametrised. However, if the Dirichlet condition does not depend on the parameter (or more precisely, on the time), it can be prescribed directly in the discrete problem without any principal changes.

\subsection{First-Order System}

Let $\left(\bar{\gamma}, \overline{\boldsymbol{u}}, \overline{\boldsymbol{\lambda}}_{D}, \overline{\boldsymbol{\lambda}}_{\nu}, \overline{\boldsymbol{\lambda}}_{\tau}\right)$ be a fixed solution of $(\mathcal{P})$. We shall now give a particular expression for the first-order system

$$
\boldsymbol{H}^{\prime}\left(\left(\bar{\gamma}, \overline{\boldsymbol{u}}, \overline{\boldsymbol{\lambda}}_{D}, \overline{\boldsymbol{\lambda}}_{\nu}, \overline{\boldsymbol{\lambda}}_{\tau}\right) ;\left(\gamma^{\prime}, \boldsymbol{u}^{\prime}, \boldsymbol{\lambda}_{D}^{\prime}, \boldsymbol{\lambda}_{\nu}^{\prime}, \boldsymbol{\lambda}_{\tau}^{\prime}\right)\right)=\mathbf{0}
$$

(recall $\left(\mathscr{P}^{\prime}\right)$ ) provided that Assumption 1 is satisfied. The dependence upon $\bar{\gamma}, \overline{\boldsymbol{u}}, \overline{\boldsymbol{\lambda}}{ }_{D}, \overline{\boldsymbol{\lambda}}_{\nu}$ and $\overline{\boldsymbol{\lambda}}_{\tau}$ will not usually be explicitly indicated in what follows.

After tedious applications of the chain rule, the first-order equilibrium equation corresponding to the first block of $\boldsymbol{H}$ reads

$$
\boldsymbol{K} \boldsymbol{u}^{\prime}-\gamma^{\prime} \boldsymbol{P}-\boldsymbol{B}_{D}^{\top} \boldsymbol{\lambda}_{D}^{\prime}-\boldsymbol{B}_{\nu}^{\top} \boldsymbol{\lambda}_{\nu}^{\prime}-\boldsymbol{B}_{\tau}^{\top} \boldsymbol{\lambda}_{\tau}^{\prime}=\mathbf{0},
$$

where we denote $\boldsymbol{B}_{\nu}=\boldsymbol{B}_{\nu}(\overline{\boldsymbol{u}}), \boldsymbol{B}_{\tau}=\boldsymbol{B}_{\tau}(\overline{\boldsymbol{u}}), \boldsymbol{P}=\frac{\partial \boldsymbol{L}}{\partial \gamma}(\bar{\gamma}, \overline{\boldsymbol{u}})$, and

$$
\boldsymbol{K}=\boldsymbol{\nabla} \boldsymbol{A}(\overline{\boldsymbol{u}})-\boldsymbol{\nabla}_{\boldsymbol{u}} \boldsymbol{L}(\bar{\gamma}, \overline{\boldsymbol{u}})-\boldsymbol{K}_{\nu}\left(\overline{\boldsymbol{u}}, \overline{\boldsymbol{\lambda}}_{\nu}\right)-\boldsymbol{K}_{\tau}\left(\overline{\boldsymbol{u}}, \overline{\boldsymbol{\lambda}}_{\tau}\right)
$$

is the $2 n_{\Omega} \times 2 n_{\Omega}$ tangent stiffness matrix with

$$
K_{\nu, i j}\left(\overline{\boldsymbol{u}}, \overline{\boldsymbol{\lambda}}_{\nu}\right)= \begin{cases}\frac{\partial \nu_{1}}{\partial x_{1}}\left(\boldsymbol{y}_{k}+\left(\bar{u}_{2 \Theta(k)-1}, \bar{u}_{2 \Theta(k)}\right)\right) \bar{\lambda}_{\nu, k} & \text { if } i=j=2 \Theta(k)-1, \\ \frac{\partial \nu_{1}}{\partial x_{2}}\left(\boldsymbol{y}_{k}+\left(\bar{u}_{2 \Theta(k)-1}, \bar{u}_{2 \Theta(k)}\right)\right) \bar{\lambda}_{\nu, k} & \text { if } i=2 \Theta(k)-1, j=2 \Theta(k), \\ \frac{\partial \nu_{2}}{\partial x_{1}}\left(\boldsymbol{y}_{k}+\left(\bar{u}_{2 \Theta(k)-1}, \bar{u}_{2 \Theta(k)}\right)\right) \bar{\lambda}_{\nu, k} & \text { if } i=2 \Theta(k), j=2 \Theta(k)-1, \\ \frac{\partial \nu_{2}}{\partial x_{2}}\left(\boldsymbol{y}_{k}+\left(\bar{u}_{2 \Theta(k)-1}, \bar{u}_{2 \Theta(k)}\right)\right) \bar{\lambda}_{\nu, k} & \text { if } i=j=2 \Theta(k), \\ 0 & \text { otherwise, }\end{cases}
$$




$$
\begin{gathered}
K_{\tau, i j}\left(\overline{\boldsymbol{u}}, \overline{\boldsymbol{\lambda}}_{\tau}\right)= \begin{cases}\frac{\partial \tau_{1}}{\partial x_{1}}\left(\boldsymbol{y}_{k}+\left(\bar{u}_{2 \Theta(k)-1}, \bar{u}_{2 \Theta(k)}\right)\right) \bar{\lambda}_{\tau, k} & \text { if } i=j=2 \Theta(k)-1, \\
\frac{\partial \tau_{1}}{\partial x_{2}}\left(\boldsymbol{y}_{k}+\left(\bar{u}_{2 \Theta(k)-1}, \bar{u}_{2 \Theta(k)}\right)\right) \bar{\lambda}_{\tau, k} & \text { if } i=2 \Theta(k)-1, j=2 \Theta(k), \\
\frac{\partial \tau_{2}}{\partial x_{1}}\left(\boldsymbol{y}_{k}+\left(\bar{u}_{2 \Theta(k)-1}, \bar{u}_{2 \Theta(k)}\right)\right) \bar{\lambda}_{\tau, k} & \text { if } i=2 \Theta(k), j=2 \Theta(k)-1, \\
\frac{\partial \tau_{2}}{\partial x_{2}}\left(\boldsymbol{y}_{k}+\left(\bar{u}_{2 \Theta(k)-1}, \bar{u}_{2 \Theta(k)}\right)\right) \bar{\lambda}_{\tau, k} & \text { if } i=j=2 \Theta(k), \\
0 & \text { otherwise, }\end{cases} \\
\frac{\partial \nu_{k}}{\partial x_{m}}=\frac{1}{\|\nabla g\|} \sum_{l=1}^{2} \frac{\partial^{2} g}{\partial x_{l} \partial x_{m}} \tau_{k} \tau_{l}, \quad \frac{\partial \tau_{k}}{\partial x_{m}}=-\frac{1}{\|\nabla g\|} \sum_{l=1}^{2} \frac{\partial^{2} g}{\partial x_{l} \partial x_{m}} \nu_{k} \tau_{l} .
\end{gathered}
$$

Remark 4. The partial derivatives of the normal and tangent are connected with the obstacle curvature $\chi$ : one has

$$
\frac{\partial \nu_{1}}{\partial x_{1}}+\frac{\partial \nu_{2}}{\partial x_{2}}=\frac{\partial \tau_{2}}{\partial x_{1}}-\frac{\partial \tau_{1}}{\partial x_{2}}=-\chi
$$

(curvature formulae for implicit planar curves can be found, for instance, in [10, Section 3]).

Furthermore, the first-order Dirichlet condition is

$$
\boldsymbol{B}_{D} \boldsymbol{u}^{\prime}-\gamma^{\prime} \boldsymbol{U}_{D}^{\prime}=\mathbf{0}
$$

with $\boldsymbol{U}_{D}^{\prime}=\boldsymbol{U}_{D}^{\prime}(\bar{\gamma})$

To derive the first-order contact conditions, one can show by other applications of the chain rule that

$$
g_{j}^{\prime}\left(\overline{\boldsymbol{u}} ; \boldsymbol{u}^{\prime}\right)=\left(\boldsymbol{B}_{\nu} \boldsymbol{u}^{\prime}\right)_{j}, \quad\left(\left(\boldsymbol{B}_{\tau}(.) .-\boldsymbol{B}_{\tau}(.) \boldsymbol{w}\right)_{j}\right)^{\prime}\left(\overline{\boldsymbol{u}} ; \boldsymbol{u}^{\prime}\right)=\left(\boldsymbol{C}_{\tau} \boldsymbol{u}^{\prime}\right)_{j},
$$

where

$$
\begin{aligned}
& \boldsymbol{C}_{\tau}= \boldsymbol{C}_{\tau}^{\overline{\boldsymbol{u}}}(\overline{\boldsymbol{u}})+\boldsymbol{B}_{\tau}-\boldsymbol{C}_{\tau}^{\boldsymbol{w}}(\overline{\boldsymbol{u}}) \in \mathbb{R}^{n_{c} \times 2 n_{\Omega}}, \\
& C_{\tau, i j}^{\boldsymbol{w}}(\overline{\boldsymbol{u}})=\left\{\begin{array}{cl}
\frac{\partial \tau_{1}}{\partial x_{1}}\left(\boldsymbol{y}_{j}+\left(\bar{u}_{2 \Theta(i)-1}, \bar{u}_{2 \Theta(i)}\right)\right) w_{2 \Theta(i)-1} \\
\quad+\frac{\partial \tau_{2}}{\partial x_{1}}\left(\boldsymbol{y}_{j}+\left(\bar{u}_{2 \Theta(i)-1}, \bar{u}_{2 \Theta(i)}\right)\right) w_{2 \Theta(i)} & \text { if } 2 \Theta(i)-1=j, \\
\frac{\partial \tau_{1}}{\partial x_{2}}\left(\boldsymbol{y}_{j}+\left(\bar{u}_{2 \Theta(i)-1}, \bar{u}_{2 \Theta(i)}\right)\right) w_{2 \Theta(i)-1} & \\
\quad+\frac{\partial \tau_{2}}{\partial x_{2}}\left(\boldsymbol{y}_{j}+\left(\bar{u}_{2 \Theta(i)-1}, \bar{u}_{2 \Theta(i)}\right)\right) w_{2 \Theta(i)} & \text { if } 2 \Theta(i)=j, \\
0 & \text { otherwise. }
\end{array}\right.
\end{aligned}
$$

Moreover, we define $P C^{1}$-functions $F: \mathbb{R}^{2} \rightarrow \mathbb{R}$ and $G_{k}: \mathbb{R}^{3} \rightarrow \mathbb{R}$ for any $k \geq 0$ by (see Figure 6)

$$
\begin{gathered}
F(a, b):=a-(a-b)_{-}= \begin{cases}a & \text { if } a-b \geq 0, \\
b & \text { if } a-b \leq 0,\end{cases} \\
G_{k}(a, b, c):=a-P_{\left[k c_{-},-k c_{-}\right]}(a-b)= \begin{cases}a & \text { if } c \geq 0, \\
b & \text { if } c \leq 0,|a-b| \leq-k c, \\
a-k c & \text { if } c \leq 0, a-b \leq k c, \\
a+k c & \text { if } c \leq 0, a-b \geq-k c .\end{cases}
\end{gathered}
$$




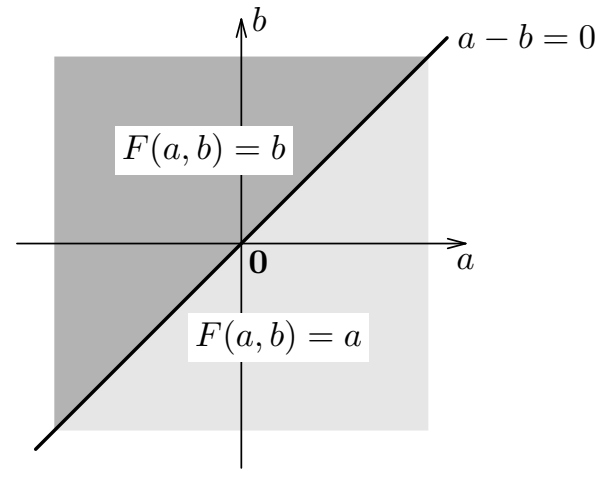

(a) $F(a, b)=a-(a-b)_{-}$.

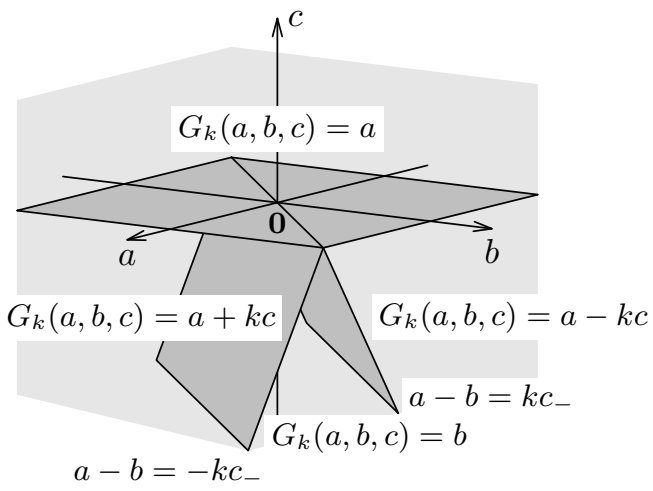

(b) $G_{k}(a, b, c)=a-P_{\left[k c_{-},-k c_{-}\right]}(a-b)$ for $k>0$.

Figure 6: Subdivisions for the $P C^{1}$-functions $F$ and $G_{k}$ defined by (23) and (24).

It is readily seen that for any $a, b, c, a^{\prime}, b^{\prime}, c^{\prime} \in \mathbb{R}$,

$$
\begin{aligned}
F^{\prime}\left((a, b) ;\left(a^{\prime}, b^{\prime}\right)\right) & = \begin{cases}a^{\prime} & \text { if } a-b>0, \\
b^{\prime} & \text { if } a-b<0, \\
a^{\prime}-\left(a^{\prime}-b^{\prime}\right)_{-} & \text {if } a-b=0,\end{cases} \\
G_{0}^{\prime}\left((a, b, c) ;\left(a^{\prime}, b^{\prime}, c^{\prime}\right)\right) & =a^{\prime}
\end{aligned}
$$

and if $k>0$,

$$
G_{k}^{\prime}\left((a, b, c) ;\left(a^{\prime}, b^{\prime}, c^{\prime}\right)\right)= \begin{cases}a^{\prime} & \text { if } c>0, \\ b^{\prime} & \text { if } c<0,|a-b|<-k c, \\ a^{\prime}-k c^{\prime} & \text { if } c<0, a-b<k c, \\ a^{\prime}+k c^{\prime} & \text { if } c<0, a-b>-k c, \\ a^{\prime}-k\left(c^{\prime}\right)_{-} & \text {if } c=0, a-b<0, \\ a^{\prime}+k\left(c^{\prime}\right)_{-} & \text {if } c=0, a-b>0, \\ a^{\prime}-P_{\left[k c^{\prime},+\infty\right)}\left(a^{\prime}-b^{\prime}\right) & \text { if } c<0, a-b=k c, \\ a^{\prime}-P_{\left(-\infty,-k c^{\prime}\right]}\left(a^{\prime}-b^{\prime}\right) & \text { if } c<0, a-b=-k c, \\ a^{\prime}-P_{\left[k\left(c^{\prime}\right)_{-},-k\left(c^{\prime}\right)_{-}\right]}\left(a^{\prime}-b^{\prime}\right) & \text { if } c=a-b=0 .\end{cases}
$$

Hence, Proposition 1 gives the first-order contact conditions in the form:

$$
\left.\begin{array}{rl}
\lambda_{\nu, j}^{\prime}=0 & \text { if } \bar{\lambda}_{\nu, j}-g_{j}(\overline{\boldsymbol{u}})>0, \\
\left(\boldsymbol{B}_{\nu} \boldsymbol{u}^{\prime}\right)_{j}=0 & \text { if } \bar{\lambda}_{\nu, j}-g_{j}(\overline{\boldsymbol{u}})<0, \\
\lambda_{\nu, j}^{\prime}-\left(\lambda_{\nu, j}^{\prime}-\left(\boldsymbol{B}_{\nu} \boldsymbol{u}^{\prime}\right)_{j}\right)_{-}=0 & \text { if } \bar{\lambda}_{\nu, j}-g_{j}(\overline{\boldsymbol{u}})=0,
\end{array}\right\}
$$




$$
\begin{aligned}
& \lambda_{\tau, j}^{\prime}=0 \quad \text { if } \mathscr{F}_{j}=0 \text { or } \mathscr{F}_{j}>0, \bar{\lambda}_{\nu, j}-g_{j}(\overline{\boldsymbol{u}})>0, \\
& \left(\boldsymbol{C}_{\tau} \boldsymbol{u}^{\prime}\right)_{j}-\gamma^{\prime} V_{\tau, j}=0 \quad \text { if } \mathscr{F}_{j}>0, \bar{\lambda}_{\nu, j}-g_{j}(\overline{\boldsymbol{u}})<0 \text {, } \\
& \left|\bar{\lambda}_{\tau, j}-\bar{v}_{\tau, j}-(1-\bar{\gamma}) V_{\tau, j}\right|<-\mathscr{F}_{j}\left(\bar{\lambda}_{\nu, j}-g_{j}(\overline{\boldsymbol{u}})\right), \\
& \lambda_{\tau, j}^{\prime}-\mathscr{F}_{j}\left(\lambda_{\nu, j}^{\prime}-\left(\boldsymbol{B}_{\nu} \boldsymbol{u}^{\prime}\right)_{j}\right)=0 \quad \text { if } \mathscr{F}_{j}>0, \bar{\lambda}_{\nu, j}-g_{j}(\overline{\boldsymbol{u}})<0 \text {, } \\
& \bar{\lambda}_{\tau, j}-\bar{v}_{\tau, j}-(1-\bar{\gamma}) V_{\tau, j}<\mathscr{F}_{j}\left(\bar{\lambda}_{\nu, j}-g_{j}(\overline{\boldsymbol{u}})\right), \\
& \lambda_{\tau, j}^{\prime}+\mathscr{F}_{j}\left(\lambda_{\nu, j}^{\prime}-\left(\boldsymbol{B}_{\nu} \boldsymbol{u}^{\prime}\right)_{j}\right)=0 \quad \text { if } \mathscr{F}_{j}>0, \bar{\lambda}_{\nu, j}-g_{j}(\overline{\boldsymbol{u}})<0, \\
& \bar{\lambda}_{\tau, j}-\bar{v}_{\tau, j}-(1-\bar{\gamma}) V_{\tau, j}>-\mathscr{F}_{j}\left(\bar{\lambda}_{\nu, j}-g_{j}(\overline{\boldsymbol{u}})\right), \\
& \lambda_{\tau, j}^{\prime}-\mathscr{F}_{j}\left(\lambda_{\nu, j}^{\prime}-\left(\boldsymbol{B}_{\nu} \boldsymbol{u}^{\prime}\right)_{j}\right)_{-}=0 \quad \text { if } \mathscr{F}_{j}>0, \bar{\lambda}_{\nu, j}-g_{j}(\overline{\boldsymbol{u}})=0 \text {, } \\
& \bar{\lambda}_{\tau, j}-\bar{v}_{\tau, j}-(1-\bar{\gamma}) V_{\tau, j}<0, \\
& \lambda_{\tau, j}^{\prime}+\mathscr{F}_{j}\left(\lambda_{\nu, j}^{\prime}-\left(\boldsymbol{B}_{\nu} \boldsymbol{u}^{\prime}\right)_{j}\right)_{-}=0 \quad \text { if } \mathscr{F}_{j}>0, \bar{\lambda}_{\nu, j}-g_{j}(\overline{\boldsymbol{u}})=0 \text {, } \\
& \bar{\lambda}_{\tau, j}-\bar{v}_{\tau, j}-(1-\bar{\gamma}) V_{\tau, j}>0, \\
& \lambda_{\tau, j}^{\prime}-P_{\left[\mathscr{F}_{j}\left(\lambda_{\nu, j}^{\prime}-\left(\boldsymbol{B}_{\nu} \boldsymbol{u}^{\prime}\right)_{j}\right)_{,+\infty}\right)}\left(\lambda_{\tau, j}^{\prime}-\left(\boldsymbol{C}_{\tau} \boldsymbol{u}^{\prime}\right)_{j}+\gamma^{\prime} V_{\tau, j}\right)=0 \\
& \text { if } \mathscr{F}_{j}>0, \bar{\lambda}_{\nu, j}-g_{j}(\overline{\boldsymbol{u}})<0, \bar{\lambda}_{\tau, j}-\bar{v}_{\tau, j}-(1-\bar{\gamma}) V_{\tau, j}=\mathscr{F}_{j}\left(\bar{\lambda}_{\nu, j}-g_{j}(\overline{\boldsymbol{u}})\right) \text {, } \\
& \lambda_{\tau, j}^{\prime}-P_{\left(-\infty,-\mathscr{F}_{j}\left(\lambda_{\nu, j}^{\prime}-\left(\boldsymbol{B}_{\nu} \boldsymbol{u}^{\prime}\right)_{j}\right)\right]}\left(\lambda_{\tau, j}^{\prime}-\left(\boldsymbol{C}_{\tau} \boldsymbol{u}^{\prime}\right)_{j}+\gamma^{\prime} V_{\tau, j}\right)=0 \\
& \text { if } \mathscr{F}_{j}>0, \bar{\lambda}_{\nu, j}-g_{j}(\overline{\boldsymbol{u}})<0, \bar{\lambda}_{\tau, j}-\bar{v}_{\tau, j}-(1-\bar{\gamma}) V_{\tau, j}=-\mathscr{F}_{j}\left(\bar{\lambda}_{\nu, j}-g_{j}(\overline{\boldsymbol{u}})\right) \text {, } \\
& \lambda_{\tau, j}^{\prime}-P_{\left[\mathscr{F}_{j}\left(\lambda_{\nu, j}^{\prime}-\left(\boldsymbol{B}_{\nu} \boldsymbol{u}^{\prime}\right)_{j}\right)_{-},-\mathscr{F}_{j}\left(\lambda_{\nu, j}^{\prime}-\left(\boldsymbol{B}_{\nu} \boldsymbol{u}^{\prime}\right)_{j}\right)_{-}\right]}\left(\lambda_{\tau, j}^{\prime}-\left(\boldsymbol{C}_{\tau} \boldsymbol{u}^{\prime}\right)_{j}+\gamma^{\prime} V_{\tau, j}\right)=0 \\
& \text { if } \mathscr{F}_{j}>0, \bar{\lambda}_{\nu, j}-g_{j}(\overline{\boldsymbol{u}})=\bar{\lambda}_{\tau, j}-\bar{v}_{\tau, j}-(1-\bar{\gamma}) V_{\tau, j}=0 \text {, }
\end{aligned}
$$

where $\bar{v}_{\tau, j}=\left(\boldsymbol{B}_{\tau}(\overline{\boldsymbol{u}}) \overline{\boldsymbol{u}}-\boldsymbol{B}_{\tau}(\overline{\boldsymbol{u}}) \boldsymbol{w}\right)_{j}$ and $j$ passes $\left\{1, \ldots, n_{c}\right\}$.

To sum up, the complete first-order system for Problem $(\mathcal{P})$ consists in finding a vector $\left(\gamma^{\prime}, \boldsymbol{u}^{\prime}, \boldsymbol{\lambda}_{D}^{\prime}, \boldsymbol{\lambda}_{\nu}^{\prime}, \boldsymbol{\lambda}_{\tau}^{\prime}\right) \in \mathbb{R}^{1+2\left(n_{\Omega}+n_{D}+n_{c}\right)}$ satisfying $\left(\mathcal{P}^{\prime} 1\right),\left(\mathcal{P}^{\prime} 2\right),\left(\mathcal{P}^{\prime} 3\right)$ and $\left(\mathcal{P}^{\prime} 4\right)$.

Unfortunately, Proposition 6 guaranteeing existence and uniqueness of solutions of a general $P C^{1}$ first-order system does not seem to be very useful for analysing this particular system. We can refer the reader to [1] and [21] for attempts to employ some results from theory of $P C^{1}$-functions to discrete contact problems. The resulting conditions include verification of signs of matrices whose number grows exponentially with the dimension of the problem. As far as we know, there is no satisfactory procedure for such a task. For this reason, we shall present some other forms of the first-order system.

To start with, we shall reformulate the contact conditions with the aid of the following lemma, which can be proved by a brief discussion. By Sgn, we denote the multifunction defined by

$$
\operatorname{Sgn}(b)= \begin{cases}\frac{b}{|b|} & \text { if } b \neq 0 \\ {[-1,1]} & \text { if } b=0\end{cases}
$$


Lemma 1. For any $a, b, c \in \mathbb{R}$ and any $k \geq 0$, it holds that

$$
\begin{aligned}
& \text { (ii) } \quad a-P_{[k c,+\infty)}(a-b)=0 \Longleftrightarrow a-k c \geq 0, b \geq 0,(a-k c) b=0 ; \\
& \text { (iii) } \quad a-P_{(-\infty,-k c]}(a-b)=0 \Longleftrightarrow a+k c \leq 0, b \leq 0,(a+k c) b=0 ; \\
& \text { (iv) } a-P_{\left[k c_{-},-k c_{-}\right]}(a-b)=0 \Longleftrightarrow a \in k c_{-} \operatorname{Sgn}(b) .
\end{aligned}
$$

Next, we introduce the index sets (compare to $\left(\mathcal{P}^{\prime} 3\right)$ and $\left.\left(\mathcal{P}^{\prime} 4\right)\right)$ :

$$
\begin{aligned}
& I=\left\{1, \ldots, n_{c}\right\}, \quad I_{\mathrm{f}}=\left\{j \in I ; \bar{\lambda}_{\nu, j}-g_{j}(\overline{\boldsymbol{u}})>0\right\}, \\
& I_{\mathrm{c}}=\left\{j \in I ; \bar{\lambda}_{\nu, j}-g_{j}(\overline{\boldsymbol{u}})<0\right\}, \quad I_{\mathrm{z}}=\left\{j \in I ; \bar{\lambda}_{\nu, j}-g_{j}(\overline{\boldsymbol{u}})=0\right\}, \\
& I_{0}=\left\{j \in I ; \mathscr{F}_{j}=0\right\} \text {, } \\
& I_{\mathrm{s}}=\left\{j \in I ; \mathscr{F}_{j}>0,\left|\bar{\lambda}_{\tau, j}-\bar{v}_{\tau, j}-(1-\bar{\gamma}) V_{\tau, j}\right|<-\mathscr{F}_{j}\left(\bar{\lambda}_{\nu, j}-g_{j}(\overline{\boldsymbol{u}})_{-}\right\},\right. \\
& I_{1}=\left\{j \in I ; \mathscr{F}_{j}>0,\left|\bar{\lambda}_{\tau, j}-\bar{v}_{\tau, j}-(1-\bar{\gamma}) V_{\tau, j}\right|>-\mathscr{F}_{j}\left(\bar{\lambda}_{\nu, j}-g_{j}(\overline{\boldsymbol{u}})\right)_{-}\right\}, \\
& I_{\mathrm{i}}=\left\{j \in I ; \mathscr{F}_{j}>0,\left|\bar{\lambda}_{\tau, j}-\bar{v}_{\tau, j}-(1-\bar{\gamma}) V_{\tau, j}\right|=-\mathscr{F}_{j}\left(\bar{\lambda}_{\nu, j}-g_{j}(\overline{\boldsymbol{u}})_{-}\right\} .\right\}
\end{aligned}
$$

Observe that the contact conditions corresponding to the last two blocks of components of $\boldsymbol{H}$ can be rewritten for $\left(\bar{\gamma}, \overline{\boldsymbol{u}}, \overline{\boldsymbol{\lambda}}_{D}, \overline{\boldsymbol{\lambda}}_{\nu}, \overline{\boldsymbol{\lambda}}_{\tau}\right)$ according to Lemma 1 as

$$
\begin{gathered}
\bar{\lambda}_{\nu, j} \leq 0, g_{j}(\overline{\boldsymbol{u}}) \leq 0, \bar{\lambda}_{\nu, j} g_{j}(\overline{\boldsymbol{u}})=0, \quad j \in I, \\
\bar{\lambda}_{\tau, j} \in \mathscr{F}_{j} \bar{\lambda}_{\nu, j} \operatorname{Sgn}\left(\bar{v}_{\tau, j}+(1-\bar{\gamma}) V_{\tau, j}\right), \quad j \in I,
\end{gathered}
$$

which can be viewed as contact conditions at each contact node. Note the modification of the friction law in virtue of the term $(1-\bar{\gamma}) V_{\tau, j}$ in comparison with the incremental problem. Although it changes slightly the physical interpretation of different contact modes, we shall use the terms "stick" and "slip" as usual in what follows, having in mind their correct interpretation.

Consequently, one obtains

$I_{\mathrm{f}}=\left\{j \in I ; \bar{\lambda}_{\nu, j}=0, g_{j}(\overline{\boldsymbol{u}})<0\right\} \quad$ (nodes not in contact - free), $I_{\mathrm{c}}=\left\{j \in I ; \bar{\lambda}_{\nu, j}<0, g_{j}(\overline{\boldsymbol{u}})=0\right\} \quad$ (nodes in strong contact),

$I_{\mathrm{z}}=\left\{j \in I ; \bar{\lambda}_{\nu, j}=g_{j}(\overline{\boldsymbol{u}})=0\right\} \quad$ (nodes in grazing contact - with zero contact forces), $I_{\mathrm{s}}=\left\{j \in I ; \mathscr{F}_{j}>0,\left|\bar{\lambda}_{\tau, j}\right|<-\mathscr{F}_{j} \bar{\lambda}_{\nu, j}, \bar{v}_{\tau, j}+(1-\bar{\gamma}) V_{\tau, j}=0\right\} \quad$ (nodes in strong stick), $I_{1}=\left\{j \in I ; \mathscr{F}_{j}>0,\left|\bar{\lambda}_{\tau, j}\right|=-\mathscr{F}_{j} \bar{\lambda}_{\nu, j}, \bar{v}_{\tau, j}+(1-\bar{\gamma}) V_{\tau, j} \neq 0\right\} \quad$ (nodes in non-zero slip), $I_{\mathrm{i}}=\left\{j \in I ; \mathscr{F}_{j}>0,\left|\bar{\lambda}_{\tau, j}\right|=-\mathscr{F}_{j} \bar{\lambda}_{\nu, j}, \bar{v}_{\tau, j}+(1-\bar{\gamma}) V_{\tau, j}=0\right\} \quad$ (nodes in impending slip)

and $I_{\mathrm{s}} \subset I_{\mathrm{c}}$.

To conclude, $\left(\mathcal{P}^{\prime} 3\right)$ and $\left(\mathcal{P}^{\prime} 4\right)$ are equivalent to

$$
\left.\begin{array}{rl}
\lambda_{\nu, j}^{\prime}=0, & j \in I_{\mathrm{f}}, \\
\left(\boldsymbol{B}_{\nu} \boldsymbol{u}^{\prime}\right)_{j}=0, & j \in I_{\mathrm{c}}, \\
\lambda_{\nu, j}^{\prime} \leq 0,\left(\boldsymbol{B}_{\nu} \boldsymbol{u}^{\prime}\right)_{j} \leq 0, \lambda_{\nu, j}^{\prime}\left(\boldsymbol{B}_{\nu} \boldsymbol{u}^{\prime}\right)_{j}=0, & j \in I_{\mathrm{z}}
\end{array}\right\}
$$




$$
\left.\begin{array}{rl}
\lambda_{\tau, j}^{\prime}=0, & j \in I_{\mathrm{f}} \cup I_{0}, \\
\left(\boldsymbol{C}_{\tau} \boldsymbol{u}^{\prime}\right)_{j}=\gamma^{\prime} V_{\tau, j}, & j \in I_{\mathrm{s}}, \\
\lambda_{\tau, j}^{\prime}=\operatorname{sgn}\left(\bar{v}_{\tau, j}+(1-\bar{\gamma}) V_{\tau, j}\right) \mathscr{F}_{j} \lambda_{\nu, j}^{\prime}, & j \in\left(I_{\mathrm{c}} \cup I_{\mathrm{z}}\right) \cap I_{1}, \\
\operatorname{sgn}\left(\bar{\lambda}_{\tau, j}\right)\left(\left(\boldsymbol{C}_{\tau} \boldsymbol{u}^{\prime}\right)_{j}-\gamma^{\prime} V_{\tau, j}\right) \leq 0, \operatorname{sgn}\left(\bar{\lambda}_{\tau, j}\right) \lambda_{\tau, j}^{\prime}+\mathscr{F}_{j} \lambda_{\nu, j}^{\prime} \leq 0, & \\
\operatorname{sgn}\left(\bar{\lambda}_{\tau, j}\right)\left(\left(\boldsymbol{C}_{\tau} \boldsymbol{u}^{\prime}\right)_{j}-\gamma^{\prime} V_{\tau, j}\right)\left(\operatorname{sgn}\left(\bar{\lambda}_{\tau, j}\right) \lambda_{\tau, j}^{\prime}+\mathscr{F}_{j} \lambda_{\nu, j}^{\prime}\right)=0, & j \in I_{\mathrm{c}} \cap I_{\mathrm{i}}, \\
\lambda_{\tau, j}^{\prime} \in \mathscr{F}_{j} \lambda_{\nu, j}^{\prime} \operatorname{Sgn}\left(\left(\boldsymbol{C}_{\tau} \boldsymbol{u}^{\prime}\right)_{j}-\gamma^{\prime} V_{\tau, j}\right), & j \in I_{\mathrm{z}} \cap I_{\mathrm{i}} .
\end{array}\right\}
$$

Mechanical interpretation of these conditions is clear: If $j \in I_{\mathrm{f}}$, normal contact stress remains zero at the $j$ th contact node, if $j \in I_{\mathrm{c}}$, the $j$ th node remains in contact with the obstacle and if $j \in I_{z}$, the node is either pressed down to the obstacle, it separates from it or it remains in grazing contact. Further, if $j \in I_{\mathrm{f}} \cup I_{0}$, friction force at the node remains zero, if $j \in I_{\mathrm{s}}$, the node remains "stuck" and if $j \in\left(I_{\mathrm{c}} \cup I_{\mathrm{z}}\right) \cap I_{\mathrm{l}}$, the tangential force remains on its bound. If $j \in I_{\mathrm{c}} \cap I_{\mathrm{i}}$, the node starts slipping, the tangential force leaves its bound or impending slip remains, and finally, if $j \in I_{\mathrm{z}} \cap I_{\mathrm{i}}$, the node obeys the full friction law.

From the mathematical point of view, the system $\left(\mathcal{P}^{\prime} 1\right),\left(\mathcal{P}^{\prime} 2\right),\left(\mathcal{P}^{\prime} 3\right.$ ') and $\left(\mathcal{P}^{\prime} 4^{\prime}\right)$ is close to a rate problem of a quasi-static contact problem discretised in space (this problem can be found in [22], for example). Let us recall that the rate problem serves for direct computing of first-order right rates of change, that is, right-hand derivatives, of a solution of a quasi-static problem for a given rate of input data. Time derivatives are not approximated contrary to our approach. Nevertheless, regarding $\gamma$ as a pseudo-time, an important difference between the two problems is that in our continuation problem, we suppress solutions in which slipping contact nodes become stuck when loading of the body changes to unloading suddenly. Indeed, there is no equivalent of the index set $I_{1}$ in the rate problem and all nodes in contact are treated either as in stick or as in possible slip in near future. Of course, this causes the rate problem to be more complicated (compare $\left(\mathcal{P}^{\prime} 4^{\prime}\right)$ for $j \in I_{1}$ with $\left.j \in I_{\mathrm{i}}\right)$.

Let us also mention that due to nodal discretisation of the contact conditions considered in this paper, the continuation problem could be formulated without the term $(1-\gamma) \boldsymbol{V}_{\tau}$ approximating a multiple of an initial velocity in the frictional condition and $\left(0, \boldsymbol{u}^{k}, \boldsymbol{\lambda}_{D}^{k}, \boldsymbol{\lambda}_{\nu}^{k}, \boldsymbol{\lambda}_{\tau}^{k}\right)$ would remain its solution (which is not always the case for other types of discretisation). However, when starting continuation from this point, one would run into an analogous situation as in the rate problem. More precisely, one would have $\overline{\boldsymbol{v}}_{\tau}=\mathbf{0}$ for $\left(\bar{\gamma}, \overline{\boldsymbol{u}}, \overline{\boldsymbol{\lambda}}_{D}, \overline{\boldsymbol{\lambda}}_{\nu}, \overline{\boldsymbol{\lambda}}_{\tau}\right)=\left(0, \boldsymbol{u}^{k}, \boldsymbol{\lambda}_{D}^{k}, \boldsymbol{\lambda}_{\nu}^{k}, \boldsymbol{\lambda}_{\tau}^{k}\right)$, the indices $j$ with $\left(\boldsymbol{B}_{\tau}\left(\boldsymbol{u}^{k}\right) \boldsymbol{u}^{k}-\boldsymbol{B}_{\tau}\left(\boldsymbol{u}^{k}\right) \boldsymbol{u}^{k-1}\right)_{j} \neq 0$, which correspond to slipping nodes at the time $t_{k}$, would fall into $I_{\mathrm{i}}$ instead of $I_{1}$ and $I_{1}$ would be empty.

Example 3. Let us consider modification of the simple example from [13, Section 4] to the quasi-static case (which is nothing else than a special case of the example with Coulomb's law of friction in [17]): contact of a single $P_{1}$ triangle with a flat foundation in the framework of small-deformation elasticity (Figure 7 ). The triangle is fixed along $\Gamma_{D}$ and the Dirichlet condition is prescribed directly in this example, hence, all degrees of freedom are related only to the vertex in $\mathbf{0}$. The volume forces and the initial state are supposed to be null. 


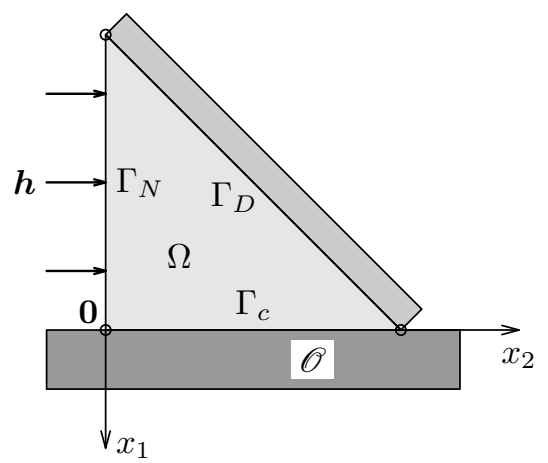

Figure 7: Geometry from Example 3.

The evolution problem can be formulated as follows (compare to $(22)$ and $(\mathcal{P})$ ):

Find $u_{1}, u_{2}, \lambda_{\nu}, \lambda_{\tau}:[0, T] \rightarrow \mathbb{R}$ such that $\left(u_{1}(0), u_{2}(0), \lambda_{\nu}(0), \lambda_{\tau}(0)\right)=(0,0,0,0)$ and for any $t \in(0, T)$, it holds that

$$
\begin{aligned}
\left(\begin{array}{cc}
a & -b \\
-b & a
\end{array}\right)\left(\begin{array}{l}
u_{1}(t) \\
u_{2}(t)
\end{array}\right)-\left(\begin{array}{l}
l_{1}(t) \\
l_{2}(t)
\end{array}\right)-\left(\begin{array}{c}
\lambda_{\nu}(t) \\
\lambda_{\tau}(t)
\end{array}\right) & =\left(\begin{array}{l}
0 \\
0
\end{array}\right), \\
\lambda_{\nu}(t)-\left(\lambda_{\nu}(t)-u_{1}(t)\right)_{-} & =0, \\
\lambda_{\tau}(t)-P_{\left[\mathscr{F}\left(\lambda_{\nu}(t)-u_{1}(t)\right)_{-},-\mathscr{F}\left(\lambda_{\nu}(t)-u_{1}(t)\right)_{-}\right]}\left(\lambda_{\tau}(t)-\dot{u}_{2}(t)\right) & =0,
\end{aligned}
$$

where $a:=(\lambda+3 \mu) / 2$ and $b:=(\lambda+\mu) / 2$ are given by Lamé constants $\lambda$ and $\mu$. The loading is considered to be $\left(l_{1}(t), l_{2}(t)\right)=(0, t)$.

According to the analysis in [17], the unique solution of this problem is

$$
u_{1}(t)=0, \quad u_{2}(t)=\frac{t}{a+\mathscr{F} b}, \quad \lambda_{\nu}(t)=-\frac{b t}{a+\mathscr{F} b}, \quad \lambda_{\tau}(t)=-\frac{\mathscr{F} b t}{a+\mathscr{F} b}
$$

and it corresponds to slip to the right of the only contact node. The rate problem at each solution point for $t>0$ is:

$$
\begin{aligned}
& \text { Find }\left(\dot{u}_{1}, \dot{u}_{2}, \dot{\lambda}_{\nu}, \dot{\lambda}_{\tau}\right) \in \mathbb{R}^{4} \text { such that } \\
& \left(\begin{array}{cc}
a & -b \\
-b & a
\end{array}\right)\left(\begin{array}{l}
\dot{u}_{1} \\
\dot{u}_{2}
\end{array}\right)-\left(\begin{array}{l}
0 \\
1
\end{array}\right)-\left(\begin{array}{l}
\dot{\lambda}_{\nu} \\
\dot{\lambda}_{\tau}
\end{array}\right)=\left(\begin{array}{l}
0 \\
0
\end{array}\right), \\
& \dot{u}_{1}=0 \text {, } \\
& \dot{u}_{2} \geq 0, \quad-\dot{\lambda}_{\tau}+\mathscr{F} \dot{\lambda}_{\nu} \leq 0, \quad \dot{u}_{2}\left(-\dot{\lambda}_{\tau}+\mathscr{F} \dot{\lambda}_{\nu}\right)=0, \quad
\end{aligned}
$$

that is, one has to deal with a complementarity between slip and stick at each time (in fact, the contact node would get stuck if the loading changed to unloading in the opposite direction).

On the other hand, solutions of the incremental problems are:

$$
u_{1}^{k}=0, \quad u_{2}^{k}=\frac{t_{k}}{a+\mathscr{F} b}, \quad \lambda_{\nu}^{k}=-\frac{b t_{k}}{a+\mathscr{F} b}, \quad \lambda_{\tau}^{k}=-\frac{\mathscr{F} b t_{k}}{a+\mathscr{F} b}
$$


and the continuation problem proposed in this paper reads as follows:

$$
\begin{aligned}
& \text { Find }\left(\gamma, u_{1}, u_{2}, \lambda_{\nu}, \lambda_{\tau}\right) \in \mathbb{R}^{5} \text { such that } \\
& \qquad \begin{array}{r}
\left(\begin{array}{cc}
a & -b \\
-b & a
\end{array}\right)\left(\begin{array}{l}
u_{1} \\
u_{2}
\end{array}\right)-\left(\begin{array}{l}
L_{1}(\gamma) \\
L_{2}(\gamma)
\end{array}\right)-\left(\begin{array}{c}
\lambda_{\nu} \\
\lambda_{\tau}
\end{array}\right)=\left(\begin{array}{l}
0 \\
0
\end{array}\right), \\
\lambda_{\nu}-\left(\lambda_{\nu}-u_{1}\right)_{-}=0,
\end{array} \\
& \begin{array}{r}
\lambda_{\tau}-P_{\left[\mathscr{F}\left(\lambda_{\nu}-u_{1}\right)_{-},-\mathscr{F}\left(\lambda_{\nu}-u_{1}\right)_{-}\right]}\left(\lambda_{\tau}-\left(u_{2}-w_{2}\right)-(1-\gamma) V_{\tau}\right)=0 .
\end{array}
\end{aligned}
$$

Here, $\left(L_{1}(\gamma), L_{2}(\gamma)\right)=\left(0, t_{k}+\gamma\left(t_{k+1}-t_{k}\right)\right), w_{2}=u_{2}^{k}$, and $V_{\tau}=\left(t_{k+1}-t_{k}\right)\left(u_{2}^{k}-u_{2}^{k-1}\right) /\left(t_{k}-\right.$ $\left.t_{k-1}\right)$ is positive for any $k \geq 1$. Solutions of this problem form the set:

$$
\begin{aligned}
&\left\{\left(\gamma, u_{1}, u_{2}, \lambda_{\nu}, \lambda_{\tau}\right) \in \mathbb{R}^{5} ; \gamma \in[0,1], u_{1}=0, u_{2}=\frac{t_{k}+\gamma\left(t_{k+1}-t_{k}\right)}{a+\mathscr{F} b}\right. \\
&\left.\lambda_{\nu}=-\frac{b\left(t_{k}+\gamma\left(t_{k+1}-t_{k}\right)\right)}{a+\mathscr{F} b}, \lambda_{\tau}=-\frac{\mathscr{F} b\left(t_{k}+\gamma\left(t_{k+1}-t_{k}\right)\right)}{a+\mathscr{F} b}\right\} .
\end{aligned}
$$

Then, the corresponding first-order system for any $\bar{\gamma} \in[0,1]$ becomes:

$$
\begin{aligned}
& \text { Find }\left(\gamma^{\prime}, u_{1}^{\prime}, u_{2}^{\prime}, \lambda_{\nu}^{\prime}, \lambda_{\tau}^{\prime}\right) \in \mathbb{R}^{5} \text { such that } \\
& \qquad \begin{array}{rc}
\left(\begin{array}{cc}
a & -b \\
-b & a
\end{array}\right)\left(\begin{array}{c}
u_{1}^{\prime} \\
u_{2}^{\prime}
\end{array}\right)-\left(\begin{array}{c}
0 \\
\gamma^{\prime}\left(t_{k+1}-t_{k}\right)
\end{array}\right)-\left(\begin{array}{c}
\lambda_{\nu}^{\prime} \\
\lambda_{\tau}^{\prime}
\end{array}\right) & =\left(\begin{array}{c}
0 \\
0
\end{array}\right) \\
u_{1}^{\prime} & =0 \\
\lambda_{\tau}^{\prime} & =\mathscr{F} \lambda_{\nu}^{\prime} .
\end{array}
\end{aligned}
$$

In comparison with the rate problem (26), the complementarity conditions are replaced by an equality here, which is apparently easier to handle.

Finally, solutions of the continuation problem without $(1-\gamma) V_{\tau}$ would remain the same as with it, however, the first-order system would contain the following frictional conditions at $\bar{\gamma}=0$ :

$$
u_{2}^{\prime} \geq 0, \quad-\lambda_{\tau}^{\prime}+\mathscr{F} \lambda_{\nu}^{\prime} \leq 0, \quad u_{2}^{\prime}\left(-\lambda_{\tau}^{\prime}+\mathscr{F} \lambda_{\nu}^{\prime}\right)=0
$$

Therefore, it would be more complicated to find a suitable initial tangent vector for numerical continuation.

Due to the similarity of the first-order system to the rate problem mentioned above, we shall follow steps in [22] and we shall reformulate it as a mixed complementarity inclusion and an explicit complementarity problem. To this end, we shall need the following assumption.

Assumption 2. Let the matrix

$$
\boldsymbol{B}:=\left(\begin{array}{c}
\boldsymbol{B}_{D} \\
\boldsymbol{B}_{\nu} \\
\boldsymbol{B}_{\tau}
\end{array}\right)
$$

have full row rank. 
One can easily verify that this assumption is equivalent to the following inf-sup condition:

$$
\exists \beta>0: \quad \inf _{\mathbf{0} \neq\left(\boldsymbol{\lambda}_{D}, \boldsymbol{\lambda}_{\nu}, \boldsymbol{\lambda}_{\tau}\right) \in \mathbb{R}^{2\left(n_{D}+n_{c}\right)}} \sup _{\mathbf{0} \neq \boldsymbol{u} \in \mathbb{R}^{2 n_{\Omega}}} \frac{\boldsymbol{u}^{\top}\left(\boldsymbol{B}_{D}^{\top} \boldsymbol{\lambda}_{D}+\boldsymbol{B}_{\nu}^{\top} \boldsymbol{\lambda}_{\nu}+\boldsymbol{B}_{\tau}^{\top} \boldsymbol{\lambda}_{\tau}\right)}{\|\boldsymbol{u}\|\left\|\left(\boldsymbol{\lambda}_{D}, \boldsymbol{\lambda}_{\nu}, \boldsymbol{\lambda}_{\tau}\right)\right\|} \geq \beta .
$$

This is a reasonable assumption on the used spatial discretisation because it prevents the discretised problem from being over-constrained. If it is satisfied,

$$
\operatorname{dim} \operatorname{Im}\left(\boldsymbol{B}^{\top}\right)=2\left(n_{D}+n_{c}\right), \quad \operatorname{dim} \operatorname{Ker}(\boldsymbol{B})=2 n_{\Omega}-2\left(n_{D}+n_{c}\right)
$$

and one can define a non-singular matrix

$$
\boldsymbol{T}_{\boldsymbol{B}}:=\left(\begin{array}{ll}
\boldsymbol{N} & \boldsymbol{B}^{+}
\end{array}\right)
$$

where the columns of the matrix $\boldsymbol{N} \in \mathbb{R}^{2 n_{\Omega} \times\left(2 n_{\Omega}-2\left(n_{D}+n_{c}\right)\right)}$ form a basis of $\operatorname{Ker}(\boldsymbol{B})$ and $\boldsymbol{B}^{+}=\boldsymbol{B}^{\top}\left(\boldsymbol{B} \boldsymbol{B}^{\top}\right)^{-1}$ is the right inverse of $\boldsymbol{B}$. Then

$$
\boldsymbol{T}_{\boldsymbol{B}}^{\top}\left(\begin{array}{lll}
\boldsymbol{B}_{D}^{\top} & \boldsymbol{B}_{\nu}^{\top} & \boldsymbol{B}_{\tau}^{\top}
\end{array}\right)\left(\begin{array}{c}
\boldsymbol{\lambda}_{D}^{\prime} \\
\boldsymbol{\lambda}_{\nu}^{\prime} \\
\boldsymbol{\lambda}_{\tau}^{\prime}
\end{array}\right)=\left(\begin{array}{ll}
\boldsymbol{B N} & \boldsymbol{B} \boldsymbol{B}^{+}
\end{array}\right)^{\top}\left(\begin{array}{c}
\boldsymbol{\lambda}_{D}^{\prime} \\
\boldsymbol{\lambda}_{\nu}^{\prime} \\
\boldsymbol{\lambda}_{\tau}^{\prime}
\end{array}\right)=\left(\begin{array}{c}
\boldsymbol{0}_{2 n_{\Omega}-\left(2 n_{D}+2 n_{c}\right)} \\
\boldsymbol{\lambda}_{D}^{\prime} \\
\boldsymbol{\lambda}_{\nu}^{\prime} \\
\boldsymbol{\lambda}_{\tau}^{\prime}
\end{array}\right) .
$$

Remark 5. For the nodal discretisation of contact conditions considered in this paper, the rows of $\left(\begin{array}{c}\boldsymbol{B}_{\nu} \\ \boldsymbol{B}_{\tau}\end{array}\right)$ are always linearly independent and they are orthogonal to the rows of $\boldsymbol{B}_{D}$. Therefore, Assumption 2 is satisfied provided that $\boldsymbol{B}_{D}$ has full row rank, which is equivalent to the inf-sup condition on the Dirichlet boundary solely:

$$
\exists \beta>0: \inf _{\mathbf{0} \neq \boldsymbol{\lambda}_{D} \in \mathbb{R}^{2 n_{D}}} \sup _{\mathbf{0} \neq \boldsymbol{u} \in \mathbb{R}^{2 n_{\Omega}}} \frac{\boldsymbol{u}^{\top} \boldsymbol{B}_{D}^{\top} \boldsymbol{\lambda}_{D}}{\|\boldsymbol{u}\|\left\|\boldsymbol{\lambda}_{D}\right\|} \geq \beta .
$$

If it is the case,

$$
\boldsymbol{T}_{\boldsymbol{B}}=\left(\begin{array}{lll}
\boldsymbol{N} & \boldsymbol{B}_{D}^{+} & \left(\begin{array}{c}
\boldsymbol{B}_{\nu} \\
\boldsymbol{B}_{\tau}
\end{array}\right)^{+}
\end{array}\right)
$$

and the columns of $\boldsymbol{N}$ and $\left(\begin{array}{c}\boldsymbol{B}_{\nu} \\ \boldsymbol{B}_{\tau}\end{array}\right)^{+}$form a basis of $\operatorname{Ker}\left(\boldsymbol{B}_{D}\right)$.

In a similar way, we shall need the following additional assumption.

Assumption 3. Let the matrix

$$
\boldsymbol{C}:=\left(\begin{array}{l}
\boldsymbol{B}_{D} \\
\boldsymbol{B}_{\nu} \\
\boldsymbol{C}_{\tau}
\end{array}\right)
$$

have full row rank. 
Let us note that if the rigid obstacle is plane, that is, $\nabla^{2} g=\mathbf{0}$, then $\boldsymbol{C}_{\tau}=\boldsymbol{B}_{\tau}$ and this assumption is always satisfied provided that Assumption 2 is fulfilled. Under Assumption 3, one can introduce a non-singular transformation matrix

$$
\boldsymbol{T}_{C}:=\left(\begin{array}{ll}
N & C^{+}
\end{array}\right)
$$

with the columns of $\boldsymbol{N}$ constituting a basis of $\operatorname{Ker}(\boldsymbol{C})$ and $\boldsymbol{C}^{+}=\boldsymbol{C}^{\top}\left(\boldsymbol{C} \boldsymbol{C}^{\top}\right)^{-1}$ (invoking the introduction of $\boldsymbol{C}$, one can easily verify that $\boldsymbol{N}$ may be taken the same as above). Consequently, any displacement direction $\boldsymbol{u}^{\prime} \in \mathbb{R}^{2 n_{\Omega}}$ in the first-order system can be represented by a vector $\tilde{\boldsymbol{u}}^{\prime}$ of the same length in the following way:

$$
\boldsymbol{u}^{\prime}=\boldsymbol{T}_{C} \tilde{\boldsymbol{u}}^{\prime}, \quad \tilde{\boldsymbol{u}}^{\prime}=\left(\begin{array}{c}
\boldsymbol{u}_{\mathrm{u}}^{\prime} \\
\boldsymbol{u}_{D}^{\prime} \\
\boldsymbol{u}_{\nu}^{\prime} \\
\boldsymbol{u}_{\tau}^{\prime}
\end{array}\right),
$$

where $\boldsymbol{u}_{D}^{\prime} \in \mathbb{R}^{2 n_{D}}$ is a part imposed to the first-order Dirichlet condition, $\boldsymbol{u}_{\nu}^{\prime}, \boldsymbol{u}_{\tau}^{\prime} \in \mathbb{R}^{n_{c}}$ correspond to the normal and tangential components on the contact zone and $\boldsymbol{u}_{\mathrm{u}}^{\prime} \in$ $\mathbb{R}^{2 n_{\Omega}-2\left(n_{D}+n_{c}\right)}$ is free of the first-order Dirichlet and contact conditions (unnconstrained). In fact,

$$
\left(\begin{array}{l}
\boldsymbol{B}_{D} \boldsymbol{u}^{\prime} \\
\boldsymbol{B}_{\nu} \boldsymbol{u}^{\prime} \\
\boldsymbol{C}_{\tau} \boldsymbol{u}^{\prime}
\end{array}\right)=\left(\begin{array}{l}
\boldsymbol{B}_{D} \\
\boldsymbol{B}_{\nu} \\
\boldsymbol{C}_{\tau}
\end{array}\right)\left(\begin{array}{ll}
\boldsymbol{N} & \boldsymbol{C}^{+}
\end{array}\right) \tilde{\boldsymbol{u}}^{\prime}=\left(\begin{array}{c}
\boldsymbol{u}_{D}^{\prime} \\
\boldsymbol{u}_{\nu}^{\prime} \\
\boldsymbol{u}_{\tau}^{\prime}
\end{array}\right) .
$$

Making use of (28) and (29) and introducing further partitioning of $\tilde{\boldsymbol{u}}^{\prime}, \boldsymbol{\lambda}_{\nu}^{\prime}$ and $\boldsymbol{\lambda}_{\tau}^{\prime}$, we can write $\left(\mathcal{P}^{\prime} 1\right)$ as

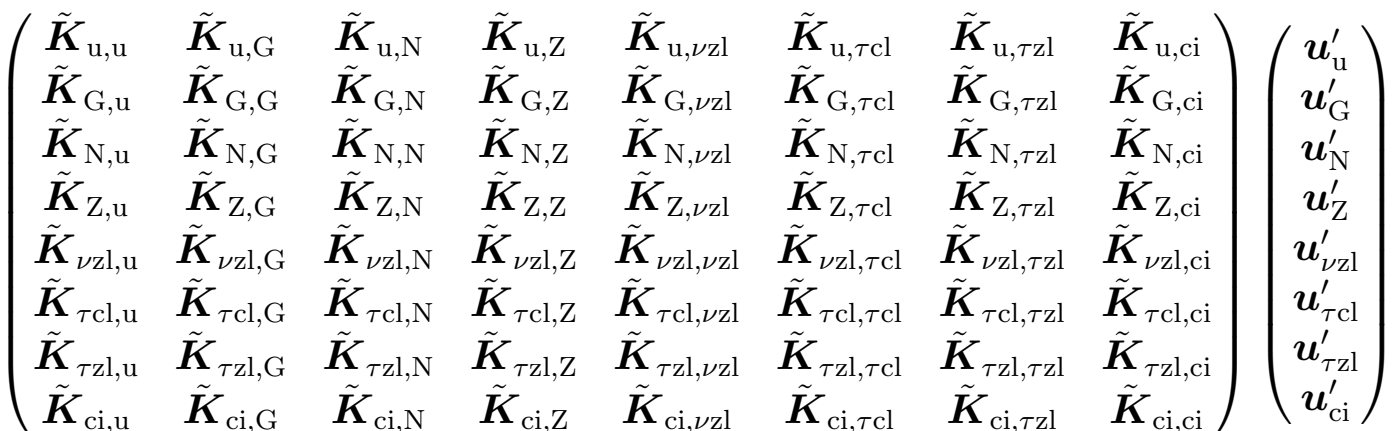

$$
\begin{aligned}
& =\left(\begin{array}{c}
\mathbf{0} \\
\boldsymbol{\lambda}_{\mathrm{G}}^{\prime} \\
\boldsymbol{\lambda}_{\mathrm{N}}^{\prime} \\
\boldsymbol{\lambda}_{\mathrm{Z}}^{\prime} \\
\boldsymbol{\lambda}_{\nu \mathrm{zl}}^{\prime} \\
\boldsymbol{\lambda}_{\tau \mathrm{cl}}^{\prime} \\
\boldsymbol{\lambda}_{\tau \mathrm{zl}}^{\prime} \\
\boldsymbol{\lambda}_{\mathrm{cl}}^{\prime}
\end{array}\right)+\gamma^{\prime}\left(\begin{array}{c}
\tilde{\boldsymbol{P}}_{\mathrm{u}} \\
\tilde{\boldsymbol{P}}_{\mathrm{G}} \\
\tilde{\boldsymbol{P}}_{\mathrm{N}} \\
\tilde{\boldsymbol{P}}_{\mathrm{Z}} \\
\tilde{\boldsymbol{P}}_{\nu \mathrm{zl}} \\
\tilde{\boldsymbol{P}}_{\tau \mathrm{cl}} \\
\tilde{\boldsymbol{P}}_{\tau \mathrm{zl}} \\
\tilde{\boldsymbol{P}}_{\mathrm{ci}}
\end{array}\right),
\end{aligned}
$$


where $\tilde{\boldsymbol{K}}=\boldsymbol{T}_{\boldsymbol{B}}^{\top} \boldsymbol{K} \boldsymbol{T}_{\boldsymbol{C}}, \tilde{\boldsymbol{P}}=\boldsymbol{T}_{\boldsymbol{B}}^{\top} \boldsymbol{P}$, G denotes jointly the indices $D, \nu \mathrm{s}, \nu \mathrm{c} 0, \nu \mathrm{cl}$ and $\tau \mathrm{s}, \mathrm{N}$ the indices $\nu \mathrm{f}, \tau \mathrm{f}, \tau \mathrm{c} 0$ and $\tau \mathrm{z} 0$, ci the indices $\nu \mathrm{ci}$ and $\tau \mathrm{ci}$, Z the indices $\nu \mathrm{z} 0, \nu \mathrm{zi}$ and $\tau \mathrm{zi}$, and $\mathrm{s}$ stands for the indices from $I_{\mathrm{s}}$, c0 for the indices from $I_{\mathrm{c}} \cap I_{0}$, cl for the indices from $I_{\mathrm{c}} \cap I_{1}$ and so forth.

The values of the components of $\boldsymbol{u}_{\mathrm{G}}^{\prime}$ and $\boldsymbol{u}_{\nu \mathrm{ci}}^{\prime}$ are given by $\left(\mathcal{P}^{\prime} 2\right),\left(\mathcal{P}^{\prime} 3^{\prime}\right)$ and $\left(\mathcal{P}^{\prime} 4^{\prime}\right)$ combined with (30):

$$
\boldsymbol{u}_{D}^{\prime}=\gamma^{\prime} \boldsymbol{U}_{D}^{\prime}, \quad \boldsymbol{u}_{\nu \mathrm{s}}^{\prime}=\mathbf{0}, \quad \boldsymbol{u}_{\nu \mathrm{c} 0}^{\prime}=\mathbf{0}, \quad \boldsymbol{u}_{\nu \mathrm{cl}}^{\prime}=\mathbf{0}, \quad \boldsymbol{u}_{\tau \mathrm{s}}^{\prime}=\gamma^{\prime} \boldsymbol{V}_{\tau \mathrm{s}}, \quad \boldsymbol{u}_{\nu \mathrm{ci}}^{\prime}=\mathbf{0}
$$

and $\boldsymbol{\lambda}_{\mathrm{G}}^{\prime}$ can be expressed directly from (31), in particular,

$$
\begin{aligned}
\boldsymbol{\lambda}_{\nu \mathrm{cl}}^{\prime}= & \tilde{\boldsymbol{K}}_{\nu \mathrm{cl}, \mathrm{u}} \boldsymbol{u}_{\mathrm{u}}^{\prime}+\tilde{\boldsymbol{K}}_{\nu \mathrm{cl}, \mathrm{N}} \boldsymbol{u}_{\mathrm{N}}^{\prime}+\tilde{\boldsymbol{K}}_{\nu \mathrm{cl}, \mathrm{Z}} \boldsymbol{u}_{\mathrm{Z}}^{\prime}+\tilde{\boldsymbol{K}}_{\nu \mathrm{cl}, \nu \mathrm{zl}} \boldsymbol{u}_{\nu \mathrm{zl}}^{\prime}+\tilde{\boldsymbol{K}}_{\nu \mathrm{cl}, \tau \mathrm{cl}} \boldsymbol{u}_{\tau \mathrm{cl}}^{\prime}+\tilde{\boldsymbol{K}}_{\nu \mathrm{cl}, \tau \mathrm{zl}} \boldsymbol{u}_{\tau \mathrm{zl}}^{\prime} \\
& +\tilde{\boldsymbol{K}}_{\nu \mathrm{cl}, \tau \mathrm{c} \mathrm{c}} \boldsymbol{u}_{\tau \mathrm{ci}}^{\prime}-\gamma^{\prime}\left(\tilde{\boldsymbol{P}}_{\nu \mathrm{cl}}-\tilde{\boldsymbol{K}}_{\nu \mathrm{cl}, D} \boldsymbol{U}_{D}^{\prime}-\tilde{\boldsymbol{K}}_{\nu \mathrm{cl}, \tau \mathrm{s}} \boldsymbol{V}_{\tau \mathrm{s}}\right) .
\end{aligned}
$$

Further, one has from $\left(\mathcal{P}^{\prime} 3^{\prime}\right)$ and $\left(\mathcal{P}^{\prime} 4^{\prime}\right)$ that

$$
\boldsymbol{\lambda}_{\mathrm{N}}^{\prime}=\mathbf{0}, \quad \boldsymbol{\lambda}_{\tau \mathrm{cl}}^{\prime}=\boldsymbol{S}_{\mathrm{cl}} \boldsymbol{F}_{\mathrm{cl}} \boldsymbol{\lambda}_{\nu \mathrm{cl}}^{\prime}, \quad \boldsymbol{\lambda}_{\tau \mathrm{zl}}^{\prime}=\boldsymbol{S}_{\mathrm{zl}} \boldsymbol{F}_{\mathrm{zl}} \boldsymbol{\lambda}_{\nu \mathrm{zl}}^{\prime},
$$

where

$$
\begin{array}{lll}
\boldsymbol{S}_{\mathrm{cl}}=\operatorname{Diag}\left(\operatorname{sgn}\left(\bar{v}_{\tau, j}+(1-\bar{\gamma}) V_{\tau, j}\right)\right), j \in I_{\mathrm{c}} \cap I_{\mathrm{l}}, & \boldsymbol{F}_{\mathrm{cl}}=\operatorname{Diag}\left(\mathscr{F}_{j}\right), j \in I_{\mathrm{c}} \cap I_{\mathrm{l}}, \\
\boldsymbol{S}_{\mathrm{zl}}=\operatorname{Diag}\left(\operatorname{sgn}\left(\bar{v}_{\tau, j}+(1-\bar{\gamma}) V_{\tau, j}\right)\right), j \in I_{\mathrm{z}} \cap I_{\mathrm{l}}, & \boldsymbol{F}_{\mathrm{zl}}=\operatorname{Diag}\left(\mathscr{F}_{j}\right), j \in I_{\mathrm{z}} \cap I_{\mathrm{l}} .
\end{array}
$$

Hence, (31) can be transformed into

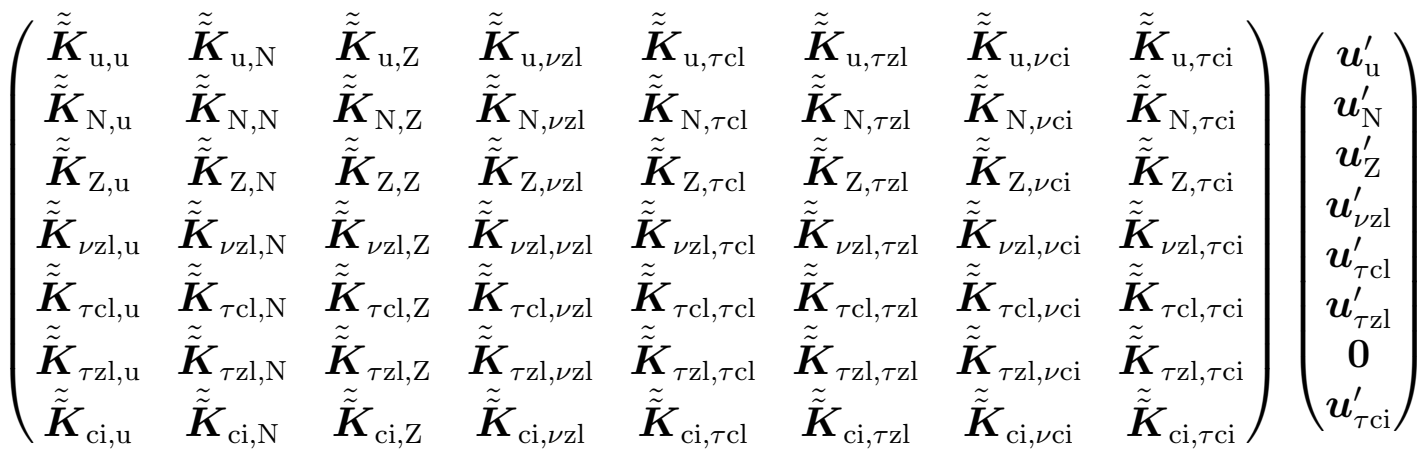

$$
\begin{aligned}
& =\left(\begin{array}{c}
\mathbf{0} \\
\mathbf{0} \\
\boldsymbol{\lambda}_{\mathrm{Z}}^{\prime} \\
\boldsymbol{\lambda}_{\nu \mathrm{zl}}^{\prime} \\
\mathbf{0} \\
\boldsymbol{S}_{\mathrm{zl}} \boldsymbol{F}_{\mathrm{zl}} \boldsymbol{\lambda}_{\nu \mathrm{zl}}^{\prime} \\
\boldsymbol{\lambda}_{\mathrm{ci}}^{\prime}
\end{array}\right)+\gamma^{\prime}\left(\begin{array}{c}
\tilde{\tilde{\boldsymbol{P}}}_{\mathrm{u}} \\
\tilde{\tilde{\boldsymbol{P}}}_{\mathrm{N}} \\
\tilde{\tilde{\boldsymbol{P}}}_{\mathrm{Z}} \\
\tilde{\tilde{\boldsymbol{P}}}_{\nu \mathrm{zl}} \\
\tilde{\tilde{\boldsymbol{P}}}_{\tau \mathrm{cl}} \\
\tilde{\tilde{\boldsymbol{P}}}_{\tau \mathrm{zl}} \\
\tilde{\tilde{\boldsymbol{P}}}_{\mathrm{ci}}
\end{array}\right)
\end{aligned}
$$


with

$$
\begin{aligned}
& \left(\begin{array}{c}
\tilde{\tilde{\boldsymbol{K}}}_{\mathrm{u}, .} \\
\tilde{\tilde{\boldsymbol{K}}}_{\mathrm{N}, .} \\
\tilde{\tilde{\boldsymbol{K}}}_{\mathrm{Z}, .} \\
\tilde{\tilde{\boldsymbol{K}}}_{\nu \mathrm{zl}, .} \\
\tilde{\tilde{\boldsymbol{K}}}_{\tau \mathrm{cl}, .} \\
\tilde{\tilde{\boldsymbol{K}}}_{\tau \mathrm{zl}, .} \\
\tilde{\tilde{\boldsymbol{K}}}_{\mathrm{ci}, .}
\end{array}\right)=\left(\begin{array}{c}
\tilde{\boldsymbol{K}}_{\mathrm{u}, .} \\
\tilde{\boldsymbol{K}}_{\mathrm{N}, .} \\
\tilde{\boldsymbol{K}}_{\mathrm{Z}, .} \\
\tilde{\boldsymbol{K}}_{\nu \mathrm{zl}, .} \\
\tilde{\boldsymbol{K}}_{\tau \mathrm{cl}, .}-\boldsymbol{S}_{\mathrm{cl}} \boldsymbol{F}_{\mathrm{cl}} \tilde{\boldsymbol{K}}_{\nu \mathrm{cl}, .} \\
\tilde{\boldsymbol{K}}_{\tau \mathrm{zl}, . .} \\
\tilde{\boldsymbol{K}}_{\mathrm{ci}, .}
\end{array}\right), \\
& \tilde{\tilde{\boldsymbol{P}}}=\left(\begin{array}{c}
\tilde{\boldsymbol{P}}_{\mathrm{u}}-\tilde{\boldsymbol{K}}_{\mathrm{u}, D} \boldsymbol{U}_{D}^{\prime}-\tilde{\boldsymbol{K}}_{\mathrm{u}, \tau \mathrm{s}} \boldsymbol{V}_{\tau \mathrm{s}} \\
\tilde{\boldsymbol{P}}_{\mathrm{N}}-\tilde{\boldsymbol{K}}_{\mathrm{N}, D} \boldsymbol{U}_{D}^{\prime}-\tilde{\boldsymbol{K}}_{\mathrm{s}, \tau \mathrm{s}} \boldsymbol{V}_{\tau \mathrm{s}} \\
\tilde{\boldsymbol{P}}_{\mathrm{Z}}-\tilde{\boldsymbol{K}}_{\mathrm{Z}, D} \boldsymbol{U}_{D}^{\prime}-\tilde{\boldsymbol{K}}_{\mathrm{Z}, \tau \mathrm{s}} \boldsymbol{V}_{\tau \mathrm{s}} \\
\tilde{\boldsymbol{P}}_{\nu \mathrm{ll}}-\tilde{\boldsymbol{K}}_{\nu \mathrm{zl}, D} \boldsymbol{U}_{D}^{\prime}-\tilde{\boldsymbol{K}}_{\nu \mathrm{l}, \tau \mathrm{s}} \boldsymbol{V}_{\tau \mathrm{s}} \\
\tilde{\boldsymbol{P}}_{\tau \mathrm{cl}}-\tilde{\boldsymbol{K}}_{\tau \mathrm{cl}, D} \boldsymbol{U}_{D}^{\prime}-\tilde{\boldsymbol{K}}_{\tau \mathrm{cl}, \tau \mathrm{s}} \boldsymbol{V}_{\tau \mathrm{s}}-\boldsymbol{S}_{\mathrm{cl}} \boldsymbol{F}_{\mathrm{cl}}\left(\tilde{\boldsymbol{P}}_{\nu \mathrm{cl}}-\tilde{\boldsymbol{K}}_{\nu \mathrm{cl}, D} \boldsymbol{U}_{D}^{\prime}-\tilde{\boldsymbol{K}}_{\nu \mathrm{cl}, \tau \mathrm{s}} \boldsymbol{V}_{\tau \mathrm{s}}\right) \\
\tilde{\boldsymbol{P}}_{\tau \mathrm{zl}}-\tilde{\boldsymbol{K}}_{\tau \mathrm{zl}, D} \boldsymbol{U}_{D}^{\prime}-\tilde{\boldsymbol{K}}_{\tau \mathrm{zl}, \tau \mathrm{s}} \boldsymbol{V}_{\tau \mathrm{s}} \\
\tilde{\boldsymbol{P}}_{\mathrm{ci}}-\tilde{\boldsymbol{K}}_{\mathrm{cl}, D} \boldsymbol{U}_{D}^{\prime}-\tilde{\boldsymbol{K}}_{\mathrm{ci}, \tau \mathrm{s}} \boldsymbol{V}_{\tau \mathrm{s}}
\end{array}\right) .
\end{aligned}
$$

Denoting the indices $\mathrm{u}, \mathrm{N}, \mathrm{Z}, \nu \mathrm{zl}, \tau \mathrm{cl}$ and $\tau$ zl jointly by E, we want to eliminate $\boldsymbol{u}_{\mathrm{E}}^{\prime}$ now. This leads us to the following assumption.

Assumption 4. Let the matrix $\tilde{\tilde{\boldsymbol{K}}}_{\mathrm{E}, \mathrm{E}}$ be invertible.

As we shall show in the proof of Corollary 1, this holds, for example, for a model of small-deformation elasticity with a properly constrained body in contact with a plane rigid obstacle provided that the coefficient of friction is sufficiently small. The assumption enables us to express $\boldsymbol{u}_{\mathrm{E}}^{\prime}$ from the first six block rows of (32):

$$
\boldsymbol{u}_{\mathrm{E}}^{\prime}=\boldsymbol{E}_{\mathrm{E}, \mathrm{Z}} \boldsymbol{\lambda}_{\mathrm{Z}}^{\prime}+\overline{\boldsymbol{E}}_{\mathrm{E}, \nu \mathrm{zl}} \boldsymbol{\lambda}_{\nu \mathrm{zl}}^{\prime}+\gamma^{\prime} \boldsymbol{E}_{\mathrm{E}, \mathrm{E}} \tilde{\widetilde{\boldsymbol{P}}}_{\mathrm{E}}-\boldsymbol{E}_{\mathrm{E}, \mathrm{E}} \tilde{\tilde{\boldsymbol{K}}}_{\mathrm{E}, \tau \mathrm{ci}} \boldsymbol{u}_{\tau \mathrm{ci}}^{\prime}
$$

where

$$
\boldsymbol{E}_{\mathrm{E}, \mathrm{E}}=\tilde{\tilde{\boldsymbol{K}}}_{\mathrm{E}, \mathrm{E}}^{-1}, \quad \overline{\boldsymbol{E}}_{\mathrm{E}, \nu \mathrm{zl}}=\boldsymbol{E}_{\mathrm{E}, \nu \mathrm{zl}}+\boldsymbol{E}_{\mathrm{E}, \tau \mathrm{zl}} \boldsymbol{S}_{\mathrm{zl}} \boldsymbol{F}_{\mathrm{zl}} .
$$

Then, substituting for it into the last block row of (32), we obtain

$$
\boldsymbol{\lambda}_{\mathrm{ci}}^{\prime}=-\gamma^{\prime} \overline{\boldsymbol{P}}_{\mathrm{ci}}+\tilde{\tilde{\boldsymbol{K}}}_{\mathrm{ci}, \mathrm{E}} \boldsymbol{E}_{\mathrm{E}, \mathrm{Z}} \boldsymbol{\lambda}_{\mathrm{Z}}^{\prime}+\tilde{\tilde{\boldsymbol{K}}}_{\mathrm{ci}, \mathrm{E}} \overline{\boldsymbol{E}}_{\mathrm{E}, \nu \mathrm{Zl}} \boldsymbol{\lambda}_{\nu \mathrm{zl}}^{\prime}+\overline{\boldsymbol{K}}_{\mathrm{ci}, \tau \mathrm{ci}} \boldsymbol{u}_{\tau \mathrm{ci}}^{\prime}
$$

with

$$
\overline{\boldsymbol{P}}_{\mathrm{ci}}=\tilde{\tilde{\boldsymbol{P}}}_{\mathrm{ci}}-\tilde{\tilde{\boldsymbol{K}}}_{\mathrm{ci}, \mathrm{E}} \boldsymbol{E}_{\mathrm{E}, \mathrm{E}} \tilde{\tilde{\boldsymbol{P}}}_{\mathrm{E}}, \quad \overline{\boldsymbol{K}}_{\mathrm{ci}, \tau \mathrm{ci}}=\tilde{\tilde{\boldsymbol{K}}}_{\mathrm{ci}, \tau \mathrm{ci}}-\tilde{\tilde{\boldsymbol{K}}}_{\mathrm{ci}, \mathrm{E}} \boldsymbol{E}_{\mathrm{E}, \mathrm{E}} \tilde{\tilde{\boldsymbol{K}}}_{\mathrm{E}, \tau \mathrm{ci}} .
$$

From here, we have

$$
\begin{aligned}
\boldsymbol{S}_{\mathrm{ci}} \boldsymbol{\lambda}_{\tau \mathrm{ci}}^{\prime} & -\boldsymbol{F}_{\mathrm{ci}} \boldsymbol{\lambda}_{\nu \mathrm{ci}}^{\prime} \\
& =-\gamma^{\prime} \overline{\overline{\boldsymbol{P}}}_{\tau \mathrm{ci}}+\overline{\overline{\boldsymbol{K}}}_{\tau \mathrm{ci}, \tau \mathrm{ci}} \boldsymbol{S}_{\mathrm{ci}}^{2}\left(\boldsymbol{u}_{\tau \mathrm{ci}}^{\prime}-\gamma^{\prime} \boldsymbol{V}_{\tau \mathrm{ci}}\right)+\overline{\overline{\boldsymbol{K}}}_{\tau \mathrm{ci}, \mathrm{E}} \boldsymbol{E}_{\mathrm{E}, \mathrm{Z}} \boldsymbol{\lambda}_{\mathrm{Z}}^{\prime}+\overline{\overline{\boldsymbol{K}}}_{\tau \mathrm{ci}, \mathrm{E}} \overline{\boldsymbol{E}}_{\mathrm{E}, \nu \mathrm{zl}} \boldsymbol{\lambda}_{\nu \mathrm{z} \mathrm{l}}^{\prime},
\end{aligned}
$$


where

$$
\begin{gathered}
\boldsymbol{S}_{\mathrm{ci}}=-\operatorname{Diag}\left(\operatorname{sgn}\left(\bar{\lambda}_{\tau, j}\right)\right), j \in I_{\mathrm{c}} \cap I_{\mathrm{i}}, \quad \boldsymbol{F}_{\mathrm{ci}}=\operatorname{Diag}\left(\mathscr{F}_{j}\right), j \in I_{\mathrm{c}} \cap I_{\mathrm{i}} \\
\overline{\overline{\boldsymbol{K}}}_{\tau \mathrm{ci}, \tau \mathrm{ci}}=\boldsymbol{S}_{\mathrm{ci}} \overline{\boldsymbol{K}}_{\tau \mathrm{ci}, \tau \mathrm{ci}}-\boldsymbol{F}_{\mathrm{ci}} \overline{\boldsymbol{K}}_{\nu \mathrm{ci}, \tau \mathrm{ci}}, \quad \overline{\overline{\boldsymbol{K}}}_{\tau \mathrm{ci}, \mathrm{E}}=\boldsymbol{S}_{\mathrm{ci}} \tilde{\tilde{\boldsymbol{K}}}_{\tau \mathrm{ci}, \mathrm{E}}-\boldsymbol{F}_{\mathrm{ci}} \tilde{\boldsymbol{K}}_{\nu \mathrm{ci}, \mathrm{E}} \\
\overline{\overline{\boldsymbol{P}}}_{\tau \mathrm{ci}}=\boldsymbol{S}_{\mathrm{ci}}\left(\overline{\boldsymbol{P}}_{\tau \mathrm{ci}}-\overline{\boldsymbol{K}}_{\tau \mathrm{ci}, \tau \mathrm{ci}} \boldsymbol{V}_{\tau \mathrm{ci}}\right)-\boldsymbol{F}_{\mathrm{ci}}\left(\overline{\boldsymbol{P}}_{\nu \mathrm{ci}}-\overline{\boldsymbol{K}}_{\nu \mathrm{ci}, \tau \mathrm{ci}} \boldsymbol{V}_{\tau \mathrm{ci}}\right) .
\end{gathered}
$$

Taking (34), (33) with the indices from $\nu \mathrm{z} 0, \nu \mathrm{zl}, \nu \mathrm{zi}$ and $\tau \mathrm{zi}$, and the remaining conditions from $\left(\mathcal{P}^{\prime} 3^{\prime}\right)$ and $\left(\mathcal{P}^{\prime} 4^{\prime}\right)$, we arrive at the mixed complementarity inclusion:

$$
\left.\begin{array}{l}
\text { Find }(\boldsymbol{x}, \boldsymbol{y}) \in \mathbb{R}^{M} \times \mathbb{R}^{M} \text { such that } \\
\boldsymbol{D} \boldsymbol{x}=\boldsymbol{y}+\gamma^{\prime} \boldsymbol{b}, \\
\boldsymbol{\zeta}_{\tau \mathrm{ci}} \geq \mathbf{0}, \boldsymbol{\psi}_{\tau \mathrm{ci}} \geq \mathbf{0}, \boldsymbol{\psi}_{\tau \mathrm{ci}}^{\top} \boldsymbol{\zeta}_{\tau \mathrm{ci}}=0, \\
\boldsymbol{\zeta}_{\nu \mathrm{z} 0} \geq \mathbf{0}, \boldsymbol{\psi}_{\nu \mathrm{z} 0} \geq \mathbf{0}, \boldsymbol{\psi}_{\nu \mathrm{z} 0}^{\top} \boldsymbol{\zeta}_{\nu \mathrm{z} 0}=0, \\
\boldsymbol{\zeta}_{\nu \mathrm{zl}} \geq \mathbf{0}, \boldsymbol{\psi}_{\nu \mathrm{zl}} \geq \mathbf{0}, \boldsymbol{\psi}_{\nu \mathrm{zl}}^{\top} \boldsymbol{\zeta}_{\nu \mathrm{zl}}=0, \\
\boldsymbol{\zeta}_{\nu \mathrm{zi}} \geq \mathbf{0}, \boldsymbol{\psi}_{\nu \mathrm{zi}} \geq \mathbf{0}, \boldsymbol{\psi}_{\nu \mathrm{zi}}^{\top} \boldsymbol{\zeta}_{\nu \mathrm{zi}}=0, \\
\boldsymbol{\psi}_{\tau \mathrm{zi}} \in-\boldsymbol{S}_{\mathrm{zi}}\left(\boldsymbol{\zeta}_{\tau \mathrm{zi}}\right) \boldsymbol{F}_{\mathrm{zi}} \boldsymbol{\psi}_{\nu \mathrm{zi}},
\end{array}\right\}
$$

where $M=n_{\mathrm{ci}}+n_{\mathrm{z} 0}+n_{\mathrm{zl}}+2 n_{\mathrm{zi}}$ with $n_{\mathrm{ci}}=\#\left(I_{\mathrm{c}} \cap I_{\mathrm{i}}\right), n_{\mathrm{z} 0}=\#\left(I_{\mathrm{z}} \cap I_{0}\right), n_{\mathrm{zl}}=\#\left(I_{\mathrm{z}} \cap I_{\mathrm{l}}\right)$ and $n_{\mathrm{zi}}=\#\left(I_{\mathrm{z}} \cap I_{\mathrm{i}}\right)$,

$$
\begin{aligned}
& \boldsymbol{x}=\left(\begin{array}{c}
\boldsymbol{\zeta}_{\tau \mathrm{ci}} \\
\boldsymbol{\psi}_{\nu \mathrm{z} 0} \\
\boldsymbol{\psi}_{\nu \mathrm{zl}} \\
\boldsymbol{\psi}_{\nu \mathrm{zi}} \\
\boldsymbol{\psi}_{\tau \mathrm{zi}}
\end{array}\right)=\left(\begin{array}{c}
\boldsymbol{S}_{\mathrm{ci}}\left(\boldsymbol{u}_{\tau \mathrm{ci}}^{\prime}-\gamma^{\prime} \boldsymbol{V}_{\tau \mathrm{ci}}\right) \\
-\boldsymbol{\lambda}_{\nu \mathrm{z} 0}^{\prime} \\
-\boldsymbol{\lambda}_{\nu \mathrm{zl}}^{\prime} \\
-\boldsymbol{\lambda}_{\nu \mathrm{zi}}^{\prime} \\
\boldsymbol{\lambda}_{\tau \mathrm{zi}}^{\prime}
\end{array}\right), \quad \boldsymbol{y}=\left(\begin{array}{c}
\boldsymbol{\psi}_{\tau \mathrm{ci}} \\
\boldsymbol{\zeta}_{\nu \mathrm{z} 0} \\
\boldsymbol{\zeta}_{\nu \mathrm{zl}} \\
\boldsymbol{\zeta}_{\nu \mathrm{zi}} \\
\boldsymbol{\zeta}_{\tau \mathrm{zi}}
\end{array}\right)=\left(\begin{array}{c}
\boldsymbol{S}_{\mathrm{ci}} \boldsymbol{\lambda}_{\tau \mathrm{ci}}^{\prime}-\boldsymbol{F}_{\mathrm{ci}} \boldsymbol{\lambda}_{\nu \mathrm{ci}}^{\prime} \\
-\boldsymbol{u}_{\nu \mathrm{z} 0}^{\prime} \\
-\boldsymbol{u}_{\nu \mathrm{zl}}^{\prime} \\
-\boldsymbol{u}_{\nu \mathrm{zi}}^{\prime} \\
\boldsymbol{u}_{\tau \mathrm{zi}}^{\prime}-\gamma^{\prime} \boldsymbol{V}_{\tau \mathrm{zi}}
\end{array}\right)
\end{aligned}
$$

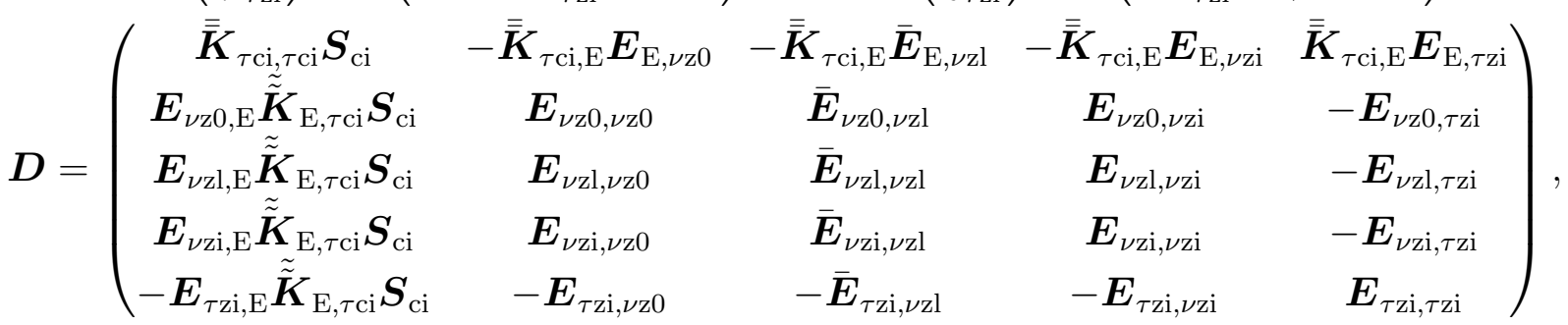

$$
\begin{aligned}
& \boldsymbol{b}=\left(\begin{array}{c}
\overline{\overline{\boldsymbol{P}}}_{\tau \mathrm{ci}} \\
\boldsymbol{E}_{\nu \mathrm{z} 0, \mathrm{E}}\left(\tilde{\tilde{\boldsymbol{P}}}_{\mathrm{E}}-\tilde{\tilde{\boldsymbol{K}}}_{\mathrm{E}, \tau \mathrm{ci}} \boldsymbol{V}_{\tau \mathrm{ci}}\right) \\
\boldsymbol{E}_{\nu \mathrm{zl}, \mathrm{E}}\left(\tilde{\tilde{\boldsymbol{P}}}_{\mathrm{E}}-\tilde{\tilde{\boldsymbol{K}}}_{\mathrm{E}, \tau \mathrm{ci}} \boldsymbol{V}_{\tau \mathrm{ci}}\right) \\
\boldsymbol{E}_{\nu \mathrm{zi}, \mathrm{E}}\left(\tilde{\tilde{\boldsymbol{P}}}_{\mathrm{E}}-\tilde{\tilde{\boldsymbol{K}}}_{\mathrm{E}, \tau \mathrm{ci}} \boldsymbol{V}_{\tau \mathrm{ci}}\right) \\
-\boldsymbol{E}_{\tau \mathrm{zi}, \mathrm{E}}\left(\tilde{\tilde{\boldsymbol{P}}}_{\mathrm{E}}-\tilde{\tilde{\boldsymbol{K}}}_{\mathrm{E}, \tau \mathrm{ci}} \boldsymbol{V}_{\tau \mathrm{ci}}\right)+\boldsymbol{V}_{\tau \mathrm{zi}}
\end{array}\right) \\
& \boldsymbol{F}_{\mathrm{zi}}=\operatorname{Diag}\left(\mathscr{F}_{j}\right), j \in I_{\mathrm{z}} \cap I_{\mathrm{i}},
\end{aligned}
$$

and $\boldsymbol{S}_{\mathrm{zi}}: \mathbb{R}^{n_{\mathrm{zi}}} \rightrightarrows \mathbb{R}^{n_{\mathrm{zi}} \times n_{\mathrm{zi}}}$ is a matrix-set-valued map defined by

$$
\boldsymbol{S}_{\mathrm{zi}}\left(\boldsymbol{\zeta}_{\tau \mathrm{zi}}\right)=\operatorname{Diag}\left(\operatorname{Sgn}\left(\left(\boldsymbol{\zeta}_{\tau \mathrm{zi}}\right)_{1}\right), \ldots, \operatorname{Sgn}\left(\left(\boldsymbol{\zeta}_{\tau \mathrm{zi}}\right)_{n_{\mathrm{zi}}}\right) .\right.
$$


It is readily seen from the construction that there is a one-to-one correspondence between solutions of the first-order system $\left(\mathcal{P}^{\prime} 1\right),\left(\mathcal{P}^{\prime} 2\right),\left(\mathcal{P}^{\prime} 3^{\prime}\right)$ and $\left(\mathcal{P}^{\prime} 4^{\prime}\right)$ and of $(35)$ for $\gamma^{\prime} \in \mathbb{R}$ given.

To transform (35) into the announced explicit complementarity problem, we start with an easy observation.

Lemma 2. For any $a, c \in \mathbb{R}$ and any $b, k \geq 0$, the following two conditions are equivalent:

$$
\begin{aligned}
& \text { (i) } a \in-k b \operatorname{Sgn}(c) \text {; } \\
& \text { (ii) }-|a|+k b \geq 0 \text { and } a c+k b|c|=0 \text {. }
\end{aligned}
$$

Next, we define closed convex cones of admissible values of $\boldsymbol{x}$ and $\boldsymbol{y}$ from (35) (all inequalities have to be understood component-wisely):

$$
\begin{gathered}
\mathscr{C}_{\boldsymbol{x}}:=\left\{\boldsymbol{x}=\left(\boldsymbol{\zeta}_{\tau \mathrm{ci}}, \boldsymbol{\psi}_{\nu \mathrm{z} 0}, \boldsymbol{\psi}_{\nu \mathrm{zl}}, \boldsymbol{\psi}_{\nu \mathrm{zi}}, \boldsymbol{\psi}_{\tau \mathrm{zi}}\right) \in \mathbb{R}^{M} ;\right. \\
\left.\boldsymbol{\zeta}_{\tau \mathrm{ci}} \geq \mathbf{0}, \boldsymbol{\psi}_{\nu \mathrm{z} 0} \geq \mathbf{0}, \boldsymbol{\psi}_{\nu \mathrm{zl}} \geq \mathbf{0}, \boldsymbol{\psi}_{\nu \mathrm{zi}} \geq \mathbf{0},-\left|\boldsymbol{\psi}_{\tau \mathrm{zi}}\right|+\boldsymbol{F}_{\mathrm{zi}} \boldsymbol{\psi}_{\nu \mathrm{zi}} \geq \mathbf{0}\right\}, \\
\mathscr{C}_{\boldsymbol{y}}:=\left\{\boldsymbol{y}=\left(\boldsymbol{\psi}_{\tau \mathrm{ci}}, \boldsymbol{\zeta}_{\nu \mathrm{z} 0}, \boldsymbol{\zeta}_{\nu \mathrm{zl}}, \boldsymbol{\zeta}_{\nu \mathrm{zi}}, \boldsymbol{\zeta}_{\tau \mathrm{zi}}\right) \in \mathbb{R}^{M} ;\right. \\
\left.\boldsymbol{\psi}_{\tau \mathrm{ci}} \geq \mathbf{0}, \boldsymbol{\zeta}_{\nu \mathrm{z} 0} \geq \mathbf{0}, \boldsymbol{\zeta}_{\nu \mathrm{zl}} \geq \mathbf{0}, \boldsymbol{\zeta}_{\nu \mathrm{zi}} \geq \mathbf{0}, \boldsymbol{\zeta}_{\tau \mathrm{zi}} \in \mathbb{R}^{n_{\mathrm{zi}}}\right\}
\end{gathered}
$$

and a mapping $\boldsymbol{h}: \mathbb{R}^{M} \rightarrow \mathbb{R}^{M}$ by

$$
\boldsymbol{h}\left(\boldsymbol{\psi}_{\tau \mathrm{ci}}, \boldsymbol{\zeta}_{\nu \mathrm{z} 0}, \boldsymbol{\zeta}_{\nu \mathrm{zl}}, \boldsymbol{\zeta}_{\nu \mathrm{zi}}, \boldsymbol{\zeta}_{\tau \mathrm{zi}}\right)=\left(\begin{array}{c}
\boldsymbol{\psi}_{\tau \mathrm{ci}} \\
\boldsymbol{\zeta}_{\nu \mathrm{z} 0} \\
\boldsymbol{\zeta}_{\nu \mathrm{zl}} \\
\boldsymbol{\zeta}_{\nu \mathrm{zi}}+\boldsymbol{F}_{\mathrm{zi}}\left|\boldsymbol{\zeta}_{\tau \mathrm{zi}}\right| \\
\boldsymbol{\zeta}_{\tau \mathrm{zi}}
\end{array}\right)
$$

It is easy to verify that these objects enjoy the properties summarised below (see also [22, Section 3]). Recall that a dual cone $\mathscr{C}^{*}$ of a cone $\mathscr{C} \subset \mathbb{R}^{M}$ is introduced as

$$
\mathscr{C}^{*}=\left\{\boldsymbol{y} \in \mathbb{R}^{M} ; \boldsymbol{y}^{\top} \boldsymbol{x} \geq 0, \forall \boldsymbol{x} \in \mathscr{C}\right\} .
$$

Lemma 3. It holds that

(i) $\boldsymbol{h}\left(\mathscr{C}_{\boldsymbol{y}}\right)=\left\{\boldsymbol{y}=\left(\boldsymbol{\psi}_{\tau \mathrm{ci}}, \boldsymbol{\zeta}_{\nu \mathrm{z} 0}, \boldsymbol{\zeta}_{\nu \mathrm{zl}}, \boldsymbol{\zeta}_{\nu \mathrm{zi}}, \boldsymbol{\zeta}_{\tau \mathrm{zi}}\right) \in \mathbb{R}^{M}\right.$;

(ii) $\boldsymbol{h}$ is a bijection.

$$
\left.\boldsymbol{\psi}_{\tau \mathrm{ci}} \geq \mathbf{0}, \boldsymbol{\zeta}_{\nu \mathrm{z} 0} \geq \mathbf{0}, \boldsymbol{\zeta}_{\nu \mathrm{zl}} \geq \mathbf{0}, \boldsymbol{\zeta}_{\nu \mathrm{zi}} \geq \mathbf{0}, \boldsymbol{\zeta}_{\nu \mathrm{zi}}-\boldsymbol{F}_{\mathrm{zi}}\left|\boldsymbol{\zeta}_{\tau \mathrm{zi}}\right| \geq \mathbf{0}\right\}=\left(\mathscr{C}_{\boldsymbol{x}}\right)^{*}
$$

Finally, define a map $\boldsymbol{f}_{\gamma^{\prime}}: \mathbb{R}^{M} \rightarrow \mathbb{R}^{M}$ by

$$
\boldsymbol{f}_{\gamma^{\prime}}(\boldsymbol{x})=\boldsymbol{h}\left(\boldsymbol{D} \boldsymbol{x}-\gamma^{\prime} \boldsymbol{b}\right)
$$

for any $\gamma^{\prime} \in \mathbb{R}$ fixed.

Altogether, it is readily seen that there is a one-to-one correspondence between solutions of (35) and the following explicit complementarity problem:

$$
\left.\begin{array}{l}
\text { Find } \boldsymbol{x} \in \mathscr{C}_{\boldsymbol{x}} \text { such that } \\
\boldsymbol{f}_{\gamma^{\prime}}(\boldsymbol{x}) \in\left(\mathscr{C}_{\boldsymbol{x}}\right)^{*} \text { and } \boldsymbol{x}^{\top} \boldsymbol{f}_{\gamma^{\prime}}(\boldsymbol{x})=0 .
\end{array}\right\}
$$




\subsubsection{Results of Existence and Uniqueness}

We are prepared to adapt the existence and uniqueness results from [22]. Following the lines on page 2804 op. cit., we immediately obtain the following:

Proposition 7. Let the matrix $\boldsymbol{D}$ be strictly co-positive in $\mathscr{C}_{\boldsymbol{x}}$, that is,

$$
\boldsymbol{x}^{\top} \boldsymbol{D} \boldsymbol{x}>0, \quad \forall \boldsymbol{x} \in \mathscr{C}_{\boldsymbol{x}}, \boldsymbol{x} \neq \mathbf{0} .
$$

Then (36) possesses a solution for any $\gamma^{\prime} \in \mathbb{R}$ and any $\boldsymbol{b} \in \mathbb{R}^{M}$.

Before rephrasing a consequence of this assertion, we recall the concept of positive definiteness of a matrix on a subspace: Let $\boldsymbol{M}$ be a square matrix of order $n$ and $V$ be an $m$-dimensional subspace of $\mathbb{R}^{n}$. We say that $\boldsymbol{M}$ is positive definite on $V$ if $\boldsymbol{u}^{\top} \boldsymbol{M} \boldsymbol{u}>0$ for any non-zero $\boldsymbol{u} \in V$, or equivalently, if the matrix $\boldsymbol{T}^{\top} \boldsymbol{M T}$ is positive definite for any matrix $\boldsymbol{T} \in \mathbb{R}^{n \times m}$ whose columns form a basis of $V$.

Corollary 1 (Existence). Let $(\mathcal{P})$ correspond to a model of small-deformation elasticity where the body is properly constrained by the Dirichlet condition, that is, the rows of $\boldsymbol{B}_{D}$ are linearly independent and the stiffness matrix $\boldsymbol{A}$ is positive definite on $\operatorname{Ker}\left(\boldsymbol{B}_{D}\right)$. If $g$ satisfies Assumption 1 and $\left\|\nabla^{2} g\right\| /\|\nabla g\|$ and $\mathscr{F}_{j}, j \in\left(\left(I_{\mathrm{c}} \cup I_{\mathrm{z}}\right) \cap I_{\mathrm{l}}\right) \cup\left(I_{\mathrm{c}} \cap I_{\mathrm{i}}\right)$, are small, then the first-order system $\left(\mathcal{P}^{\prime} 1\right),\left(\mathcal{P}^{\prime} 2\right),\left(\mathcal{P}^{\prime} 3^{\prime}\right)$ and $\left(\mathcal{P}^{\prime} 4{ }^{\prime}\right)$ has at least one solution for any $\gamma^{\prime} \in \mathbb{R}, \boldsymbol{P} \in \mathbb{R}^{2 n_{\Omega}}$ and $\boldsymbol{U}_{D}^{\prime} \in \mathbb{R}^{2 n_{D}}$.

Proof. Suppose for a while that the assumptions are satisfied even with $\nabla^{2} g=\mathbf{0}$ and $\mathscr{F}_{j}=0$ for $j \in\left(\left(I_{\mathrm{c}} \cup I_{\mathrm{z}}\right) \cap I_{\mathrm{l}}\right) \cup\left(I_{\mathrm{c}} \cap I_{\mathrm{i}}\right)$. Then $\boldsymbol{K}=\boldsymbol{A}, \boldsymbol{C}_{\tau}=\boldsymbol{B}_{\tau}$ and both Assumptions

2 and 3 hold according to Remark 5 and the assumption on $\boldsymbol{B}_{D}$. Furthermore, $\tilde{\tilde{\boldsymbol{K}}}$ is a principal sub-matrix of $\boldsymbol{N}_{D}^{\top} \boldsymbol{K} \boldsymbol{N}_{D}$, where the columns of $\boldsymbol{N}_{D}$ are formed by the columns of $\boldsymbol{N}$ and $\left(\begin{array}{c}\boldsymbol{B}_{\nu} \\ \boldsymbol{B}_{\tau}\end{array}\right)^{+}$constituting a basis of $\operatorname{Ker}\left(\boldsymbol{B}_{D}\right)$. Thus, $\tilde{\tilde{\boldsymbol{K}}}$ is symmetric positive definite and so are $\tilde{\tilde{\boldsymbol{K}}}_{E, E}$ and its inverse $\boldsymbol{E}_{\mathrm{E}, \mathrm{E}}$. Consequently, Assumption 4 is fulfilled, the first-order system can be transformed into (36) by the procedure described above and

$$
\begin{aligned}
\boldsymbol{x}^{\top} \boldsymbol{D} \boldsymbol{x}= & \boldsymbol{\zeta}_{\tau \mathrm{ci}}^{\top} \boldsymbol{S}_{\mathrm{ci}} \overline{\boldsymbol{K}}_{\tau \mathrm{ci}, \tau \mathrm{ci}} \boldsymbol{S}_{\mathrm{ci}} \boldsymbol{\zeta}_{\tau \mathrm{ci}} \\
& +\left(\begin{array}{llll}
\boldsymbol{\psi}_{\nu \mathrm{z} 0}^{\top} & \boldsymbol{\psi}_{\nu \mathrm{zl}}^{\top} & \boldsymbol{\psi}_{\nu \mathrm{zi}}^{\top} & -\boldsymbol{\psi}_{\tau \mathrm{zi}}^{\top}
\end{array}\right)\left(\begin{array}{llll}
\boldsymbol{E}_{\nu \mathrm{z} 0, \nu \mathrm{z} 0} & \boldsymbol{E}_{\nu \mathrm{z} 0, \nu \mathrm{zl}} & \boldsymbol{E}_{\nu \mathrm{z} 0, \nu \mathrm{zi}} & \boldsymbol{E}_{\nu \mathrm{z} 0, \tau \mathrm{zi}} \\
\boldsymbol{E}_{\nu \mathrm{zl}, \nu \mathrm{z} 0} & \boldsymbol{E}_{\nu \mathrm{zl}, \nu \mathrm{zl}} & \boldsymbol{E}_{\nu \mathrm{zl}, \nu \mathrm{zi}} & \boldsymbol{E}_{\nu \mathrm{zl}, \tau \mathrm{zi}} \\
\boldsymbol{E}_{\nu \mathrm{zi}, \nu \mathrm{z} 0} & \boldsymbol{E}_{\nu \mathrm{zi}, \nu \mathrm{zl}} & \boldsymbol{E}_{\nu \mathrm{zi}, \nu \mathrm{zi}} & \boldsymbol{E}_{\nu \mathrm{zi}, \tau \mathrm{zi}} \\
\boldsymbol{E}_{\tau \mathrm{zi}, \nu \mathrm{z} 0} & \boldsymbol{E}_{\tau \mathrm{zi}, \nu \mathrm{zl}} & \boldsymbol{E}_{\tau \mathrm{zi}, \nu \mathrm{zi}} & \boldsymbol{E}_{\tau \mathrm{zi}, \tau \mathrm{zi}}
\end{array}\right)\left(\begin{array}{c}
\boldsymbol{\psi}_{\nu \mathrm{z} 0} \\
\boldsymbol{\psi}_{\nu \mathrm{zl}} \\
\boldsymbol{\psi}_{\nu \mathrm{zi}} \\
-\boldsymbol{\psi}_{\tau \mathrm{zi}}
\end{array}\right) .
\end{aligned}
$$

The matrix $\boldsymbol{S}_{\mathrm{ci}} \overline{\boldsymbol{K}}_{\tau \mathrm{ci}, \tau \mathrm{ci}} \boldsymbol{S}_{\mathrm{ci}}=\boldsymbol{S}_{\mathrm{ci}}^{-1} \overline{\boldsymbol{K}}_{\tau \mathrm{ci}, \tau \mathrm{ci}} \boldsymbol{S}_{\mathrm{ci}}$ in the first term above is similar to $\overline{\boldsymbol{K}}_{\tau \mathrm{ci}, \tau \mathrm{ci}}=$ $\tilde{\tilde{\boldsymbol{K}}}_{\tau \mathrm{ci}, \tau \mathrm{ci}}-\tilde{\tilde{\boldsymbol{K}}}_{\tau \mathrm{ci}, \mathrm{E}} \boldsymbol{E}_{\mathrm{E}, \mathrm{E}} \tilde{\boldsymbol{\boldsymbol { K }}}_{\mathrm{E}, \tau \mathrm{ci}}$, which is a principal sub-matrix of the Schur complement of $\tilde{\tilde{\boldsymbol{K}}}_{\mathrm{E}, \mathrm{E}}$ in $\tilde{\tilde{\boldsymbol{K}}}$. Thus, it is positive definite and so is the principal sub-matrix of $\boldsymbol{E}$ in the second term above. This implies the positive definiteness of $\boldsymbol{D}$, a fortiori fulfilment of (37) and the previous proposition yields the assertion under the stronger assumptions on $g$ and $\mathscr{F}_{j}$.

Continuous dependence of the elements of $\boldsymbol{C}_{\tau}, \tilde{\tilde{\boldsymbol{K}}}$ and $\boldsymbol{D}$ on the elements of $\nabla^{2} g /\|\nabla g\|$ and $\mathscr{F}_{j}, j \in\left(\left(I_{\mathrm{c}} \cup I_{\mathrm{z}}\right) \cap I_{\mathrm{l}}\right) \cup\left(I_{\mathrm{c}} \cap I_{\mathrm{i}}\right)$, completes the claim. 
The uniqueness result, analogous to [22, Proposition 5], uses the notion of a $P$-matrix, which is a matrix whose all principal minors are positive.

Proposition 8. A sufficient condition for uniqueness of a solution of (35) for $\gamma^{\prime} \in \mathbb{R}$ and $\boldsymbol{b} \in \mathbb{R}^{M}$ given is that the matrix

$$
\boldsymbol{D}\left(\begin{array}{ccccc}
\boldsymbol{I} & \mathbf{0} & \mathbf{0} & \mathbf{0} & \mathbf{0} \\
\mathbf{0} & \boldsymbol{I} & \mathbf{0} & \mathbf{0} & \mathbf{0} \\
\mathbf{0} & \mathbf{0} & \boldsymbol{I} & \mathbf{0} & \mathbf{0} \\
\mathbf{0} & \mathbf{0} & \mathbf{0} & \boldsymbol{I} & \mathbf{0} \\
\mathbf{0} & \mathbf{0} & \mathbf{0} & -\tilde{\boldsymbol{S}}_{\mathrm{zi}} \boldsymbol{F}_{\mathrm{zi}} & \boldsymbol{I}
\end{array}\right)
$$

is a P-matrix for any $\tilde{\boldsymbol{S}}_{\mathrm{zi}}=\operatorname{Diag}\left(\tilde{s}_{1}, \ldots, \tilde{s}_{n_{\mathrm{zi}}}\right)$ with $\tilde{s}_{j} \in[-1,1]$.

Finally, invoking that a symmetric positive definite matrix is a $P$-matrix and arguing as for Corollary 1, we get the following:

Corollary 2 (Uniqueness). Let $(\mathcal{P})$ correspond to a model of small-deformation elasticity where the body is properly constrained by the Dirichlet condition. If $g$ satisfies Assumption 1 and $\left\|\nabla^{2} g\right\| /\|\nabla g\|$ and $\mathscr{F}_{j}, j \in\left(I_{\mathrm{c}} \cup I_{\mathrm{z}}\right) \cap\left(I_{1} \cup I_{\mathrm{i}}\right)$, are small, then the first-order system $\left(\mathcal{P}^{\prime} 1\right),\left(\mathcal{P}^{\prime} 2\right),\left(\mathcal{P}^{\prime} 3\right.$ ') and $\left(\mathcal{P}^{\prime} 4\right.$ ') has a unique solution for any $\gamma^{\prime} \in \mathbb{R}, \boldsymbol{P} \in \mathbb{R}^{2 n_{\Omega}}$ and any $\boldsymbol{U}_{D}^{\prime} \in \mathbb{R}^{2 n_{D}}$.

Example 4 (Large coefficient of friction). (i) Let us consider the simple continuation problem (27) from Example 3 with $\mathscr{F}=a / b,\left(L_{1}(\gamma), L_{2}(\gamma)\right)=(1,-\gamma)$ and $w_{2}=V_{\tau}=0$.

Taking $\left(\bar{\gamma}, \bar{u}_{1}, \bar{u}_{2}, \bar{\lambda}_{\nu}, \bar{\lambda}_{\tau}\right)=(\mathscr{F}, 0,0,-1, \mathscr{F})$ (strong contact with impending slip), we get the first-order system:

$$
\begin{gathered}
\left(\begin{array}{cc}
a & -b \\
-b & a
\end{array}\right)\left(\begin{array}{l}
u_{1}^{\prime} \\
u_{2}^{\prime}
\end{array}\right)-\gamma^{\prime}\left(\begin{array}{l}
P_{1} \\
P_{2}
\end{array}\right)-\left(\begin{array}{l}
\lambda_{\nu}^{\prime} \\
\lambda_{\tau}^{\prime}
\end{array}\right)=\left(\begin{array}{l}
0 \\
0
\end{array}\right), \\
u_{1}^{\prime}=0, \quad u_{2}^{\prime} \leq 0, \lambda_{\tau}^{\prime}+\mathscr{F} \lambda_{\nu}^{\prime} \leq 0, \quad u_{2}^{\prime}\left(\lambda_{\tau}^{\prime}+\mathscr{F} \lambda_{\nu}^{\prime}\right)=0
\end{gathered}
$$

with $\left(P_{1}, P_{2}\right)=(0,-1)$. Its solutions form the sets

$$
\begin{aligned}
\left\{\left(\gamma^{\prime}, u_{1}^{\prime}, u_{2}^{\prime}, \lambda_{\nu}^{\prime}, \lambda_{\tau}^{\prime}\right) \in \mathbb{R}^{5} ;\right. & \left(\gamma^{\prime} \leq 0, u_{1}^{\prime}=u_{2}^{\prime}=0, \lambda_{\nu}^{\prime}=0, \lambda_{\tau}^{\prime}=\gamma^{\prime}\right) \\
& \left.\vee\left(\gamma^{\prime}=0, u_{1}^{\prime}=0, u_{2}^{\prime} \leq 0, \lambda_{\nu}^{\prime}=-b u_{2}^{\prime}, \lambda_{\tau}^{\prime}=\mathscr{F} b u_{2}^{\prime}\right)\right\},
\end{aligned}
$$

that is, there is no solution for $\gamma^{\prime}>0$ but infinity of solutions for $\gamma^{\prime}=0$.

The first-order system for $\left(\bar{\gamma}, \bar{u}_{1}, \bar{u}_{2}, \bar{\lambda}_{\nu}, \bar{\lambda}_{\tau}\right)=(\mathscr{F}, 0, \beta,-1-b \beta, \mathscr{F}(1+b \beta)), \beta \in$ $(-1 / b, 0)$ (strong contact with positive slip) consists of $(38)$ and

$$
u_{1}^{\prime}=0, \lambda_{\tau}^{\prime}+\mathscr{F} \lambda_{\nu}^{\prime}=0
$$

and its solution set is

$$
\left\{\left(\gamma^{\prime}, u_{1}^{\prime}, u_{2}^{\prime}, \lambda_{\nu}^{\prime}, \lambda_{\tau}^{\prime}\right) \in \mathbb{R}^{5} ; \gamma^{\prime}=0, u_{1}^{\prime}=0, u_{2}^{\prime} \in \mathbb{R}, \lambda_{\nu}^{\prime}=-b u_{2}^{\prime}, \lambda_{\tau}^{\prime}=\mathscr{F} b u_{2}^{\prime}\right\} .
$$


It has no solution for $\gamma^{\prime} \neq 0$ but infinity of solutions for $\gamma^{\prime}=0$.

Finally, the first-order system for $\left(\bar{\gamma}, \bar{u}_{1}, \bar{u}_{2}, \bar{\lambda}_{\nu}, \bar{\lambda}_{\tau}\right)=(\mathscr{F}, 0,-1 / b, 0,0)$ (grazing contact with positive slip) is composed of (38) and

$$
u_{1}^{\prime} \leq 0, \lambda_{\nu}^{\prime} \leq 0, u_{1}^{\prime} \lambda_{\nu}^{\prime}=0, \lambda_{\tau}^{\prime}+\mathscr{F} \lambda_{\nu}^{\prime}=0
$$

and its solutions are

$$
\begin{aligned}
\left\{\left(\gamma^{\prime}, u_{1}^{\prime}, u_{2}^{\prime}, \lambda_{\nu}^{\prime}, \lambda_{\tau}^{\prime}\right) \in \mathbb{R}^{5} ;\right. & \left(\gamma^{\prime}=0, u_{1}^{\prime}=0, u_{2}^{\prime} \geq 0, \lambda_{\nu}^{\prime}=-b u_{2}^{\prime}, \lambda_{\tau}^{\prime}=\mathscr{F} b u_{2}^{\prime}\right) \\
\vee & \left.\left(\gamma^{\prime} \geq 0, u_{1}^{\prime}=\frac{-b \gamma^{\prime}}{a^{2}-b^{2}}, u_{2}^{\prime}=\frac{-a \gamma^{\prime}}{a^{2}-b^{2}}, \lambda_{\nu}^{\prime}=\lambda_{\tau}^{\prime}=0\right)\right\} .
\end{aligned}
$$

There is no solution for $\gamma^{\prime}<0$ but infinity of solutions for $\gamma^{\prime}=0$.

(ii) Now, let us consider Problem (27) with $\mathscr{F}=a / b,\left(L_{1}(\gamma), L_{2}(\gamma)\right)=(b \gamma,-a \gamma)$ and $w_{2}=V_{\tau}=0$ and choose $\left(\bar{\gamma}, \bar{u}_{1}, \bar{u}_{2}, \bar{\lambda}_{\nu}, \bar{\lambda}_{\tau}\right)=(0,0,0,0,0)$ (grazing contact with impending slip). In this case, the first-order system comprises (38) with $\left(P_{1}, P_{2}\right)=(b,-a)$ and

$$
u_{1}^{\prime} \leq 0, \lambda_{\nu}^{\prime} \leq 0, u_{1}^{\prime} \lambda_{\nu}^{\prime}=0, \lambda_{\tau}^{\prime} \in \mathscr{F} \lambda_{\nu}^{\prime} \operatorname{Sgn}\left(u_{2}^{\prime}\right) .
$$

One can find the following solution set for $\gamma^{\prime} \geq 0$ :

$$
\begin{aligned}
\left\{\left(\gamma^{\prime}, u_{1}^{\prime}, u_{2}^{\prime}, \lambda_{\nu}^{\prime}, \lambda_{\tau}^{\prime}\right) \in\right. & \mathbb{R}^{5} ;\left(\gamma^{\prime} \geq 0, u_{1}^{\prime}=u_{2}^{\prime}=0, \lambda_{\nu}^{\prime}=-b \gamma^{\prime}, \lambda_{\tau}^{\prime}=a \gamma^{\prime}\right) \\
& \vee\left(\gamma^{\prime} \geq 0, u_{1}^{\prime}=0, u_{2}^{\prime} \in\left[-\gamma^{\prime}, 0\right], \lambda_{\nu}^{\prime}=-b\left(\gamma^{\prime}+u_{2}^{\prime}\right), \lambda_{\tau}^{\prime}=\mathscr{F} b\left(\gamma^{\prime}+u_{2}^{\prime}\right)\right) \\
& \left.\vee\left(\gamma^{\prime} \geq 0, u_{1}^{\prime}=0, u_{2}^{\prime}=-\gamma^{\prime}, \lambda_{\nu}^{\prime}=\lambda_{\tau}^{\prime}=0\right)\right\} .
\end{aligned}
$$

It consists of a continuous branch of solutions for any $\gamma^{\prime}>0$ fixed.

To sum up, we see that the first-order system can lack solutions for some $\gamma^{\prime}$ if $\mathscr{F}_{j}$ are large for $j \in I_{\mathrm{c}} \cap I_{\mathrm{i}}, j \in I_{\mathrm{c}} \cap I_{\mathrm{l}}$, or $j \in I_{\mathrm{z}} \cap I_{\mathrm{l}}$. On the other hand, it can possess multiple solutions for some $\gamma^{\prime}$ if $\mathscr{F}_{j}$ are large for $j \in I_{\mathrm{c}} \cap I_{\mathrm{i}}, j \in I_{\mathrm{c}} \cap I_{\mathrm{l}}, j \in I_{\mathrm{z}} \cap I_{\mathrm{l}}$ or $j \in I_{\mathrm{z}} \cap I_{\mathrm{i}}$.

Example 5 (Large $\left\|\nabla^{2} g\right\| /\|\nabla g\|$ ). Let us modify the simple continuation problem (27) to the case where the rigid foundation is demarcated by

$$
g: \boldsymbol{x}=\left(x_{1}, x_{2}\right) \mapsto r^{2}-\left(x_{1}-r\right)^{2}-x_{2}^{2}
$$

for $r>0$ fixed (Figure 8). Then

$$
\boldsymbol{\nu}(\boldsymbol{x})=\frac{-1}{\sqrt{\left(x_{1}-r\right)^{2}+x_{2}^{2}}}\left(\begin{array}{c}
x_{1}-r \\
x_{2}
\end{array}\right), \quad \boldsymbol{\tau}(\boldsymbol{x})=\frac{1}{\sqrt{\left(x_{1}-r\right)^{2}+x_{2}^{2}}}\left(\begin{array}{c}
x_{2} \\
-\left(x_{1}-r\right)
\end{array}\right)
$$

and the continuation problem becomes

$$
\begin{aligned}
\left(\begin{array}{cc}
a & -b \\
-b & a
\end{array}\right) \boldsymbol{u}-\left(\begin{array}{c}
L_{1}(\gamma) \\
L_{2}(\gamma)
\end{array}\right)-\lambda_{\nu} \boldsymbol{\nu}(\boldsymbol{u})-\lambda_{\tau} \boldsymbol{\tau}(\boldsymbol{u}) & =\left(\begin{array}{c}
0 \\
0
\end{array}\right), \\
\lambda_{\nu}-\left(\lambda_{\nu}-g(\boldsymbol{u})\right)_{-} & =0, \\
\lambda_{\tau}-P_{\left[\mathscr{F}\left(\lambda_{\nu}-g(\boldsymbol{u})\right)_{-}, \mathscr{F}\left(\lambda_{\nu}-g(\boldsymbol{u})\right)_{-}\right]}\left(\lambda_{\tau}-\left(\boldsymbol{\tau}(\boldsymbol{u})^{\top} \boldsymbol{u}-\boldsymbol{\tau}(\boldsymbol{u})^{\top} \boldsymbol{w}\right)-(1-\gamma) V_{\tau}\right) & =0 .
\end{aligned}
$$




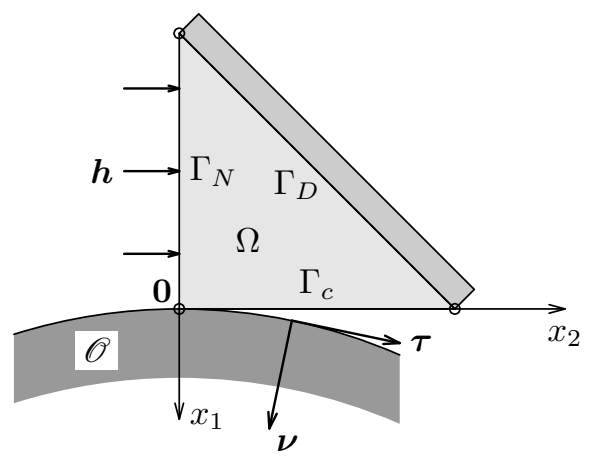

Figure 8: Geometry of Example 5.

Here, we consider $\left(L_{1}(\gamma), L_{2}(\gamma)\right)=(1,-\gamma), \boldsymbol{w}=\mathbf{0}, V_{\tau}=0$ and $\mathscr{F}>0$ arbitrarily fixed.

The first-order system for $\left(\bar{\gamma}, \bar{u}_{1}, \bar{u}_{2}, \bar{\lambda}_{\nu}, \bar{\lambda}_{\tau}\right)=(\mathscr{F}, 0,0,-1, \mathscr{F})$ (strong contact with impending slip) reads:

$$
\begin{gathered}
\left(\begin{array}{cc}
a & -b-\frac{\mathscr{F}}{r} \\
-b & a-\frac{1}{r}
\end{array}\right)\left(\begin{array}{l}
u_{1}^{\prime} \\
u_{2}^{\prime}
\end{array}\right)+\left(\begin{array}{c}
0 \\
\gamma^{\prime}
\end{array}\right)-\left(\begin{array}{c}
\lambda_{\nu}^{\prime} \\
\lambda_{\tau}^{\prime}
\end{array}\right)=\left(\begin{array}{l}
0 \\
0
\end{array}\right), \\
u_{1}^{\prime}=0, \quad u_{2}^{\prime} \leq 0, \lambda_{\tau}^{\prime}+\mathscr{F} \lambda_{\nu}^{\prime} \leq 0, u_{2}^{\prime}\left(\lambda_{\tau}^{\prime}+\mathscr{F} \lambda_{\nu}^{\prime}\right)=0 .
\end{gathered}
$$

Now, if $r$ is small enough so that $a-b \mathscr{F}-\left(1+\mathscr{F}^{2}\right) / r<0$, its solution set is

$$
\begin{aligned}
\left\{\left(\gamma^{\prime}, u_{1}^{\prime}, u_{2}^{\prime}, \lambda_{\nu}^{\prime}, \lambda_{\tau}^{\prime}\right) \in \mathbb{R}^{5} ;\left(\gamma^{\prime} \leq 0, u_{1}^{\prime}=u_{2}^{\prime}=0, \lambda_{\nu}^{\prime}=0, \lambda_{\tau}^{\prime}=\gamma^{\prime}\right)\right. & \\
\vee & \left(\gamma^{\prime} \leq 0, u_{1}^{\prime}=0, u_{2}^{\prime}=\frac{-\gamma^{\prime}}{a-b \mathscr{F}-\left(1+\mathscr{F}^{2}\right) / r},\right. \\
& \left.\left.\lambda_{\nu}^{\prime}=\frac{(b+\mathscr{F} / r) \gamma^{\prime}}{a-b \mathscr{F}-\left(1+\mathscr{F}^{2}\right) / r}, \lambda_{\tau}^{\prime}=\frac{-\mathscr{F}(b+\mathscr{F} / r) \gamma^{\prime}}{a-b \mathscr{F}-\left(1+\mathscr{F}^{2}\right) / r}\right)\right\} .
\end{aligned}
$$

Hence, this first-order system has no solution for $\gamma^{\prime}>0$ and two different solutions for $\gamma^{\prime}<0$. This shows that large $\left\|\nabla^{2} g\right\| /\|\nabla g\|$ can cause both lack and multiplicity of solutions for some $\gamma^{\prime}$.

Let us point out that the first-order system can lack solutions for some $\gamma^{\prime}$ for larger coefficients of friction even in the case of small-deformation elasticity with the body properly constrained by the Dirichlet condition and in contact with a flat obstacle. This is in stark contrast to the corresponding incremental problem, where existence of a solution is guaranteed for any coefficient of friction [18, Section 6]. On the other hand, it is in accordance with the analysis of the rate and quasi-static problem. It was shown in $[22$, Subsection 5.2] that solvability of the rate problem requires smallness of the coefficient of friction for nodes in strong contact with possible slip in near future and an example of non-existence of solutions of a quasi-static problem for high values of the coefficient of friction can be found in [17].

To add, the uniqueness result for the rate problem presented in [22] involves an assumption on smallness of the coefficient of friction for nodes in contact with possible slip 
in near future and for nodes in grazing contact. In [17], an example with multiple solutions of a quasi-static problem with high values of the coefficient of friction was shown.

Finally, let us note that the analogue in [22] of our assumption on the smallness of $\left\|\nabla^{2} g\right\| /\|\nabla g\|$ is an assumption on smallness of the obstacle curvature $\chi$ in the case that it is positive (which corresponds to a convex obstacle). It is not clear to us whether the same assumption would be sufficient also in our analysis and, in particular, whether one could distinguish between convex and concave obstacles.

\subsubsection{Continuation in a Direction Solving the First-Order System}

To show the possibility of continuation of a solution curve in a direction solving the firstorder system, we shall apply Theorem 2 from the abstract frame.

Let $\left(\bar{\gamma}, \overline{\boldsymbol{u}}, \overline{\boldsymbol{\lambda}}_{D}, \overline{\boldsymbol{\lambda}}_{\nu}, \overline{\boldsymbol{\lambda}}_{\tau}\right)(=(\bar{\gamma}, \overline{\boldsymbol{x}}))$ be a fixed solution of $(\mathcal{P})$, that is, $\boldsymbol{H}\left(\bar{\gamma}, \overline{\boldsymbol{u}}, \overline{\boldsymbol{\lambda}}_{D}, \overline{\boldsymbol{\lambda}}_{\nu}, \overline{\boldsymbol{\lambda}}_{\tau}\right)=$ $\mathbf{0}$, and $I_{\mathrm{f}}, I_{\mathrm{c}}, I_{\mathrm{z}}, I_{0}, I_{\mathrm{s}}, I_{\mathrm{l}}, I_{\mathrm{i}}$ be the corresponding index sets defined by (25). First of all, we shall construct selection functions of $\boldsymbol{H}$ at $\left(\bar{\gamma}, \overline{\boldsymbol{u}}, \overline{\boldsymbol{\lambda}}_{D}, \overline{\boldsymbol{\lambda}}_{\nu}, \overline{\boldsymbol{\lambda}}_{\tau}\right)$ in a similar spirit as in [5].

Consider $\left(\gamma, \boldsymbol{u}, \boldsymbol{\lambda}_{D}, \boldsymbol{\lambda}_{\nu}, \boldsymbol{\lambda}_{\tau}\right)$ from a neighbourhood of $\left(\bar{\gamma}, \overline{\boldsymbol{u}}, \overline{\boldsymbol{\lambda}}_{D}, \overline{\boldsymbol{\lambda}}_{\nu}, \overline{\boldsymbol{\lambda}}_{\tau}\right)$. For each $j \in I_{\mathrm{z}}$, one of the following two possibilities occurs:

$$
\begin{aligned}
& \lambda_{\nu, j}-g_{j}(\boldsymbol{u}) \geq 0, \\
& \lambda_{\nu, j}-g_{j}(\boldsymbol{u}) \leq 0 .
\end{aligned}
$$

In a similar way, there are two possibilities for each $j \in I_{\mathrm{c}} \cap I_{\mathrm{i}}$ :

$$
\begin{aligned}
& \operatorname{sgn}\left(\bar{\lambda}_{\tau, j}\right)\left(\lambda_{\tau, j}-\left(\boldsymbol{B}_{\tau}(\boldsymbol{u}) \boldsymbol{u}-\boldsymbol{B}_{\tau}(\boldsymbol{u}) \boldsymbol{w}\right)_{j}-(1-\gamma) V_{\tau, j}\right) \leq-\mathscr{F}_{j}\left(\lambda_{\nu, j}-g_{j}(\boldsymbol{u})\right), \\
& \operatorname{sgn}\left(\bar{\lambda}_{\tau, j}\right)\left(\lambda_{\tau, j}-\left(\boldsymbol{B}_{\tau}(\boldsymbol{u}) \boldsymbol{u}-\boldsymbol{B}_{\tau}(\boldsymbol{u}) \boldsymbol{w}\right)_{j}-(1-\gamma) V_{\tau, j}\right) \geq-\mathscr{F}_{j}\left(\lambda_{\nu, j}-g_{j}(\boldsymbol{u})\right) .
\end{aligned}
$$

Finally, if $j \in I_{\mathrm{z}} \cap I_{\mathrm{i}}$ and (40) occurs, the following three further cases are possible:

$$
\begin{array}{r}
\left|\left(\lambda_{\tau, j}-\left(\boldsymbol{B}_{\tau}(\boldsymbol{u}) \boldsymbol{u}-\boldsymbol{B}_{\tau}(\boldsymbol{u}) \boldsymbol{w}\right)_{j}-(1-\gamma) V_{\tau, j}\right)\right| \leq-\mathscr{F}_{j}\left(\lambda_{\nu, j}-g_{j}(\boldsymbol{u})\right), \\
-\left(\lambda_{\tau, j}-\left(\boldsymbol{B}_{\tau}(\boldsymbol{u}) \boldsymbol{u}-\boldsymbol{B}_{\tau}(\boldsymbol{u}) \boldsymbol{w}\right)_{j}-(1-\gamma) V_{\tau, j}\right) \geq-\mathscr{F}_{j}\left(\lambda_{\nu, j}-g_{j}(\boldsymbol{u})\right), \\
\lambda_{\tau, j}-\left(\boldsymbol{B}_{\tau}(\boldsymbol{u}) \boldsymbol{u}-\boldsymbol{B}_{\tau}(\boldsymbol{u}) \boldsymbol{w}\right)_{j}-(1-\gamma) V_{\tau, j} \geq-\mathscr{F}_{j}\left(\lambda_{\nu, j}-g_{j}(\boldsymbol{u})\right) .
\end{array}
$$

In this way, one obtains a decomposition of $I_{\mathrm{z}}$ into two subsets, say $I_{\mathrm{z}}^{\mathrm{f}}$ and $I_{\mathrm{z}}^{\mathrm{c}}$, defined by (39) and (40), respectively, a decomposition of $I_{\mathrm{c}} \cap I_{\mathrm{i}}$ into other two subsets, say $I_{\text {ci }}^{\mathrm{s}}$ and $I_{\text {ci }}^{\mathrm{l}}$, defined by (41) and (42), respectively, and a further decomposition of $I_{\mathrm{z}}^{\mathrm{c}} \cap I_{\mathrm{i}}$ into $I_{\mathrm{zi}}^{\mathrm{cs}}, I_{\mathrm{zi}}^{\mathrm{cl}+}$ and $I_{\mathrm{zi}}^{\mathrm{cl}-}$ given by (43), (44) and (45), respectively. Let us mention that if $\left(\gamma, \boldsymbol{u}, \boldsymbol{\lambda}_{D}, \boldsymbol{\lambda}_{\nu}, \boldsymbol{\lambda}_{\tau}\right)$ happens to be a zero of $\boldsymbol{H}$, then (39) and (40) correspond to no contact and contact, respectively, (41) and (42) to stick and slip, respectively, and (43), (44) and (45) to stick, positive and negative slip, respectively.

Now associate the obtained index sets with a function $\boldsymbol{H}^{(i)}: \mathbb{R}^{1+2\left(n_{\Omega}+n_{D}+n_{c}\right)} \rightarrow \mathbb{R}^{2\left(n_{\Omega}+n_{D}+n_{c}\right)}$, $i=i\left(I_{\mathrm{z}}^{\mathrm{f}}, I_{\mathrm{z}}^{\mathrm{c}}, I_{\mathrm{ci}}^{\mathrm{s}}, I_{\mathrm{ci}}^{\mathrm{l}}, I_{\mathrm{zi}}^{\mathrm{cs}}, I_{\mathrm{zi}}^{\mathrm{cl}+}, I_{\mathrm{zi}}^{\mathrm{cl}-}\right)$ (we do not indicate the dependence upon $I_{\mathrm{f}}, I_{\mathrm{c}}, I_{\mathrm{z}}, I_{0}, I_{\mathrm{s}}$, 
$I_{1}$ and $I_{\mathrm{i}}$ since these are considered to be fixed by $\left.\left(\bar{\gamma}, \overline{\boldsymbol{u}}, \overline{\boldsymbol{\lambda}}_{D}, \overline{\boldsymbol{\lambda}}_{\nu}, \overline{\boldsymbol{\lambda}}_{\tau}\right)\right)$, introduced by:

$$
\begin{aligned}
& \boldsymbol{H}^{(i)}\left(\gamma, \boldsymbol{u}, \boldsymbol{\lambda}_{D}, \boldsymbol{\lambda}_{\nu}, \boldsymbol{\lambda}_{\tau}\right) \\
& =\left(\begin{array}{c}
\boldsymbol{A}(\boldsymbol{u})-\boldsymbol{L}(\gamma, \boldsymbol{u})-\boldsymbol{B}_{D}^{\top} \boldsymbol{\lambda}_{D}-\boldsymbol{B}_{\nu}^{\top}(\boldsymbol{u}) \boldsymbol{\lambda}_{\nu}-\boldsymbol{B}_{\tau}^{\top}(\boldsymbol{u}) \boldsymbol{\lambda}_{\tau} \\
\boldsymbol{B}_{D} \boldsymbol{u}-\boldsymbol{U}_{D}(\gamma) \\
\left(\begin{array}{cc}
\lambda_{\nu, j}, & j \in I_{\mathrm{f}} \cup I_{\mathrm{z}}^{\mathrm{f}} \\
g_{j}(\boldsymbol{u}), \quad j \in I_{\mathrm{c}} \cup I_{\mathrm{z}}^{\mathrm{c}}
\end{array}\right) \\
\lambda_{\tau, j}, j \in I_{\mathrm{f}} \cup I_{\mathrm{z}}^{\mathrm{f}} \cup I_{0} \\
\left(\begin{array}{c} 
\\
\left(\boldsymbol{B}_{\tau}(\boldsymbol{u}) \boldsymbol{u}-\boldsymbol{B}_{\tau}(\boldsymbol{u}) \boldsymbol{w}\right)_{j}+(1-\gamma) V_{\tau, j}, \quad j \in I_{\mathrm{s}} \cup I_{\mathrm{ci}}^{\mathrm{s}} \cup I_{\mathrm{zi}}^{\mathrm{cs}} \\
\lambda_{\tau, j}-s_{j} \mathscr{F}_{j}\left(\lambda_{\nu, j}-g_{j}(\boldsymbol{u})\right), \quad j \in\left(I_{\mathrm{c}} \cap I_{\mathrm{l}}\right) \cup I_{\mathrm{zl}}^{\mathrm{c}} \cup I_{\mathrm{ci}}^{\mathrm{c}} \cup I_{\mathrm{zi}}^{\mathrm{cl}} \cup I_{\mathrm{zi}}^{\mathrm{cl}-}
\end{array}\right)
\end{array}\right),
\end{aligned}
$$

where $I_{\mathrm{zl}}^{\mathrm{c}}=I_{\mathrm{z}}^{\mathrm{c}} \cap I_{\mathrm{l}}$ and

$$
s_{j}= \begin{cases}\operatorname{sgn}\left(\left(\boldsymbol{B}_{\tau}(\overline{\boldsymbol{u}}) \overline{\boldsymbol{u}}-\boldsymbol{B}_{\tau}(\overline{\boldsymbol{u}}) \boldsymbol{w}\right)_{j}+(1-\bar{\gamma}) V_{\tau, j}\right) & \text { if } j \in\left(I_{\mathrm{c}} \cap I_{\mathrm{l}}\right) \cup I_{\mathrm{zl}}^{\mathrm{c}}, \\ -\operatorname{sgn}\left(\bar{\lambda}_{\tau, j}\right) & \text { if } j \in I_{\mathrm{ci}}^{\mathrm{l}}, \\ \pm 1 & \text { if } j \in I_{\mathrm{zi}}^{\mathrm{cl} \pm}\end{cases}
$$

(compare to the functions $F$ and $G_{k}$ in (23) and (24)). If $\left(\gamma, \boldsymbol{u}, \boldsymbol{\lambda}_{D}, \boldsymbol{\lambda}_{\nu}, \boldsymbol{\lambda}_{\tau}\right)$ was chosen sufficiently close to $\left(\bar{\gamma}, \overline{\boldsymbol{u}}, \overline{\boldsymbol{\lambda}}_{D}, \overline{\boldsymbol{\lambda}}_{\nu}, \overline{\boldsymbol{\lambda}}_{\tau}\right)$, the continuity of $\boldsymbol{H}$ ensures that

$$
\boldsymbol{H}\left(\gamma, \boldsymbol{u}, \boldsymbol{\lambda}_{D}, \boldsymbol{\lambda}_{\nu}, \boldsymbol{\lambda}_{\tau}\right)=\boldsymbol{H}^{(i)}\left(\gamma, \boldsymbol{u}, \boldsymbol{\lambda}_{D}, \boldsymbol{\lambda}_{\nu}, \boldsymbol{\lambda}_{\tau}\right)
$$

This leads us to the following introduction of the set of selection functions of $\boldsymbol{H}$ at $\left(\bar{\gamma}, \overline{\boldsymbol{u}}, \overline{\boldsymbol{\lambda}}_{D}, \overline{\boldsymbol{\lambda}}_{\nu}, \overline{\boldsymbol{\lambda}}_{\tau}\right)$ : we take the set $\left\{\boldsymbol{H}^{(i)}\right\}$ corresponding to all combinations of $I_{\mathrm{z}}^{\mathrm{f}}$ and $I_{\mathrm{z}}^{\mathrm{c}}$ forming a decomposition of $I_{\mathrm{z}}, I_{\mathrm{ci}}^{\mathrm{s}}$ and $I_{\mathrm{ci}}^{\mathrm{l}}$ forming a decomposition of $I_{\mathrm{c}} \cap I_{\mathrm{i}}$, and $I_{\mathrm{zi}}^{\mathrm{cs}}, I_{\mathrm{zi}}^{\mathrm{cl}+}$ and $I_{\mathrm{zi}}^{\mathrm{cl}-}$ forming a decomposition of $I_{\mathrm{z}}^{\mathrm{c}} \cap I_{\mathrm{i}}$.

Invoking the derivation of $\left(\mathcal{P}^{\prime} 1\right)-\left(\mathcal{P}^{\prime} 4\right)$, one can see that the corresponding gradients satisfy

$$
\left.\begin{array}{rl}
\boldsymbol{\nabla} \boldsymbol{H}^{(i)}\left(\bar{\gamma}, \overline{\boldsymbol{u}}, \overline{\boldsymbol{\lambda}}_{D}, \overline{\boldsymbol{\lambda}}_{\nu}, \overline{\boldsymbol{\lambda}}_{\tau}\right)\left(\gamma^{\prime}, \boldsymbol{u}^{\prime}, \boldsymbol{\lambda}_{D}^{\prime}, \boldsymbol{\lambda}_{\nu}^{\prime}, \boldsymbol{\lambda}_{\tau}^{\prime}\right) \\
\boldsymbol{K} \boldsymbol{u}^{\prime}-\gamma^{\prime} \boldsymbol{P}-\boldsymbol{B}_{D}^{\top} \boldsymbol{\lambda}_{D}^{\prime}-\boldsymbol{B}_{\nu}^{\top} \boldsymbol{\lambda}_{\nu}^{\prime}-\boldsymbol{B}_{\tau}^{\top} \boldsymbol{\lambda}_{\tau}^{\prime} \\
\boldsymbol{B}_{D} \boldsymbol{u}^{\prime}-\gamma^{\prime} \boldsymbol{U}_{D}^{\prime} \\
\left(\begin{array}{c}
\lambda_{\nu, j}^{\prime}, \quad j \in I_{\mathrm{f}} \cup I_{\mathrm{z}}^{\mathrm{f}} \\
\left(\boldsymbol{B}_{\nu} \boldsymbol{u}^{\prime}\right)_{j}, \quad j \in I_{\mathrm{c}} \cup I_{\mathrm{z}}^{\mathrm{c}}
\end{array}\right) \\
\lambda_{\tau, j}^{\prime}, j \in I_{\mathrm{f}} \cup I_{\mathrm{z}}^{\mathrm{f}} \cup I_{0} \\
\left(\begin{array}{c}
\left.\boldsymbol{C}_{\tau} \boldsymbol{u}^{\prime}\right)_{j}-\gamma^{\prime} V_{\tau, j}, \quad j \in I_{\mathrm{s}} \cup I_{\mathrm{ci}}^{\mathrm{s}} \cup I_{\mathrm{zi}}^{\mathrm{cs}} \\
\left(\boldsymbol{B}_{\nu} \boldsymbol{u}_{j}^{\prime}\right), \quad j \in\left(I_{\mathrm{c}} \cap I_{\mathrm{l}}\right) \cup I_{\mathrm{zl}}^{\mathrm{c}} \cup I_{\mathrm{ci}}^{\mathrm{l}} \cup I_{\mathrm{zi}}^{\mathrm{cl}+} \cup I_{\mathrm{zi}}^{\mathrm{cl}-}
\end{array}\right)
\end{array}\right)
$$

for any $\left(\gamma^{\prime}, \boldsymbol{u}^{\prime}, \boldsymbol{\lambda}_{D}^{\prime}, \boldsymbol{\lambda}_{\nu}^{\prime}, \boldsymbol{\lambda}_{\tau}^{\prime}\right) \in \mathbb{R}^{1+2\left(n_{\Omega}+n_{D}+n_{c}\right)}$. Furthermore, we have

$$
\boldsymbol{H}^{\prime}\left(\left(\bar{\gamma}, \overline{\boldsymbol{u}}, \overline{\boldsymbol{\lambda}}_{D}, \overline{\boldsymbol{\lambda}}_{\nu}, \overline{\boldsymbol{\lambda}}_{\tau}\right) ;\left(\gamma^{\prime}, \boldsymbol{u}^{\prime}, \boldsymbol{\lambda}_{D}^{\prime}, \boldsymbol{\lambda}_{\nu}^{\prime}, \boldsymbol{\lambda}_{\tau}^{\prime}\right)\right)=\nabla \boldsymbol{H}^{(i)}\left(\bar{\gamma}, \overline{\boldsymbol{u}}, \overline{\boldsymbol{\lambda}}_{D}, \overline{\boldsymbol{\lambda}}_{\nu}, \overline{\boldsymbol{\lambda}}_{\tau}\right)\left(\gamma^{\prime}, \boldsymbol{u}^{\prime}, \boldsymbol{\lambda}_{D}^{\prime}, \boldsymbol{\lambda}_{\nu}^{\prime}, \boldsymbol{\lambda}_{\tau}^{\prime}\right)
$$


if

$$
\left.\begin{array}{cl}
\lambda_{\nu, j}^{\prime}-\left(\boldsymbol{B}_{\nu} \boldsymbol{u}^{\prime}\right)_{j} \geq 0, & j \in I_{\mathrm{z}}^{\mathrm{f}}, \\
\lambda_{\nu, j}^{\prime}-\left(\boldsymbol{B}_{\nu} \boldsymbol{u}^{\prime}\right)_{j} \leq 0, & j \in I_{\mathrm{z}}^{\mathrm{c}}, \\
-s_{j}\left(\lambda_{\tau, j}^{\prime}-\left(\boldsymbol{C}_{\tau} \boldsymbol{u}^{\prime}\right)_{j}+\gamma^{\prime} V_{\tau, j}\right) \leq-\mathscr{F}_{j}\left(\lambda_{\nu, j}^{\prime}-\left(\boldsymbol{B}_{\nu} \boldsymbol{u}^{\prime}\right)_{j}\right), \quad j \in I_{\mathrm{ci}}^{\mathrm{s}}, \\
\left|\lambda_{\tau, j}^{\prime}-\left(\boldsymbol{C}_{\tau} \boldsymbol{u}^{\prime}\right)_{j}+\gamma^{\prime} V_{\tau, j}\right| \leq-\mathscr{F}_{j}\left(\lambda_{\nu, j}^{\prime}-\left(\boldsymbol{B}_{\nu} \boldsymbol{u}^{\prime}\right)_{j}\right), \quad j \in I_{\mathrm{zi}}^{\mathrm{cs}}, \\
-s_{j}\left(\lambda_{\tau, j}^{\prime}-\left(\boldsymbol{C}_{\tau} \boldsymbol{u}^{\prime}\right)_{j}+\gamma^{\prime} V_{\tau, j}\right) \geq-\mathscr{F}_{j}\left(\lambda_{\nu, j}^{\prime}-\left(\boldsymbol{B}_{\nu} \boldsymbol{u}^{\prime}\right)_{j}\right), \quad j \in I_{\mathrm{ci}}^{1} \cup I_{\mathrm{zi}}^{\mathrm{cl}+} \cup I_{\mathrm{zi}}^{\mathrm{cl}-},
\end{array}\right\}
$$

with $s_{j}$ given by (46) and

$$
s_{j}=-\operatorname{sgn}\left(\bar{\lambda}_{\tau, j}\right) \quad \text { if } j \in I_{\mathrm{ci}}^{\mathrm{s}} .
$$

We are prepared to state the continuation result based on Theorem 2 and Remark 2.

Theorem 3. Let Assumption 1 be satisfied, $\left(\bar{\gamma}, \overline{\boldsymbol{u}}, \overline{\boldsymbol{\lambda}}_{D}, \overline{\boldsymbol{\lambda}}_{\nu}, \overline{\boldsymbol{\lambda}}_{\tau}\right)$ be a solution of $(\mathcal{P})$ with non-empty $I_{\mathrm{z}} \cup\left(I_{\mathrm{c}} \cap I_{\mathrm{i}}\right)$, and $\left(\gamma^{\prime}, \boldsymbol{u}^{\prime}, \boldsymbol{\lambda}_{D}^{\prime}, \boldsymbol{\lambda}_{\nu}^{\prime}, \boldsymbol{\lambda}_{\tau}^{\prime}\right)$ solve the first-order system $\left(\mathcal{P}^{\prime} 1\right),\left(\mathcal{P}^{\prime} 2\right)$, $\left(\mathcal{P}^{\prime} 3^{\prime}\right)$ and $\left(\mathcal{P}^{\prime} 4^{\prime}\right)$. If there are index sets $I_{\mathrm{z}}^{\mathrm{f}}$ and $I_{\mathrm{z}}^{\mathrm{c}}, I_{\mathrm{ci}}^{\mathrm{s}}$ and $I_{\mathrm{ci}}^{\mathrm{l}}$, and $I_{\mathrm{zi}}^{\mathrm{cs}}$ and $I_{\mathrm{zi}}^{\mathrm{cl}}$ constituting decompositions of $I_{\mathrm{z}}, I_{\mathrm{c}} \cap I_{\mathrm{i}}$ and $I_{\mathrm{z}}^{\mathrm{c}} \cap I_{\mathrm{i}}$, respectively, and such that

(i) all the inequalities in (49) are satisfied in strict sense;

(ii) the gradient of the corresponding selection function $\boldsymbol{H}^{(i)}$ at $\left(\bar{\gamma}, \overline{\boldsymbol{u}}, \overline{\boldsymbol{\lambda}}_{D}, \overline{\boldsymbol{\lambda}}_{\nu}, \overline{\boldsymbol{\lambda}}_{\tau}\right)$ given by (48) has the maximal rank,

then there exist $\delta>0$ and a $C^{1}$-curve $\boldsymbol{c}:[\bar{s}, \bar{s}+\delta) \rightarrow \mathbb{R}^{1+2\left(n_{\Omega}+n_{D}+n_{c}\right)}$ such that

$$
\begin{gathered}
\text { (j) } \boldsymbol{c}(\bar{s})=\left(\bar{\gamma}, \overline{\boldsymbol{u}}, \overline{\boldsymbol{\lambda}}_{D}, \overline{\boldsymbol{\lambda}}_{\nu}, \overline{\boldsymbol{\lambda}}_{\tau}\right) ; \\
(\mathrm{jj}) \boldsymbol{H}(\boldsymbol{c}(s))=\mathbf{0}, \forall s \in[\bar{s}, \bar{s}+\delta) \\
(\mathrm{jjj}) \boldsymbol{c}^{\prime}(\bar{s})=\left(\gamma^{\prime}, \boldsymbol{u}^{\prime}, \boldsymbol{\lambda}_{D}^{\prime}, \boldsymbol{\lambda}_{\nu}^{\prime}, \boldsymbol{\lambda}_{\tau}^{\prime}\right) .
\end{gathered}
$$

Moreover, the image of any other curve $\tilde{\boldsymbol{c}}:[\bar{s}, \bar{s}+\tilde{\delta}) \rightarrow \mathbb{R}^{1+2\left(n_{\Omega}+n_{D}+n_{c}\right)}$ with $\tilde{\delta}>0$ and such that

$$
\left.\begin{array}{r}
(\widetilde{\mathrm{j}}) \tilde{\boldsymbol{c}}(\bar{s})=\left(\bar{\gamma}, \overline{\boldsymbol{u}}, \overline{\boldsymbol{\lambda}}_{D}, \overline{\boldsymbol{\lambda}}_{\nu}, \overline{\boldsymbol{\lambda}}_{\tau}\right) ; \\
(\widetilde{\mathrm{jj}}) \boldsymbol{H}(\tilde{\boldsymbol{c}}(s))=\mathbf{0}, \forall s \in[\bar{s}, \bar{s}+\tilde{\delta}) ; \\
(\widetilde{\mathrm{jjj}}) \tilde{\boldsymbol{c}}^{\prime}(\bar{s}) \neq \mathbf{0} \text { satisfies }(49)
\end{array}\right\}
$$

coincides with the image of $\boldsymbol{c}$ around $\left(\bar{\gamma}, \overline{\boldsymbol{u}}, \overline{\boldsymbol{\lambda}}_{D}, \overline{\boldsymbol{\lambda}}_{\nu}, \overline{\boldsymbol{\lambda}}_{\tau}\right)$.

Proof. Let $\left(\bar{\gamma}, \overline{\boldsymbol{u}}, \overline{\boldsymbol{\lambda}}_{D}, \overline{\boldsymbol{\lambda}}_{\nu}, \overline{\boldsymbol{\lambda}}_{\tau}\right)$ and $\left(\gamma^{\prime}, \boldsymbol{u}^{\prime}, \boldsymbol{\lambda}_{D}^{\prime}, \boldsymbol{\lambda}_{\nu}^{\prime}, \boldsymbol{\lambda}_{\tau}^{\prime}\right)$ meet the imposed assumptions and $\boldsymbol{H}^{\left(i_{0}\right)}$ be the selection function corresponding to the particular index sets from the assertion. We shall verify the assumptions of Theorem 2 .

It is clear from the considerations that all selection functions constructed above are active at $\left(\bar{\gamma}, \overline{\boldsymbol{u}}, \overline{\boldsymbol{\lambda}}_{D}, \overline{\boldsymbol{\lambda}}_{\nu}, \overline{\boldsymbol{\lambda}}_{\tau}\right)$, in particular,

$$
\boldsymbol{H}^{\left(i_{0}\right)}\left(\bar{\gamma}, \overline{\boldsymbol{u}}, \overline{\boldsymbol{\lambda}}_{D}, \overline{\boldsymbol{\lambda}}_{\nu}, \overline{\boldsymbol{\lambda}}_{\tau}\right)=\boldsymbol{H}\left(\bar{\gamma}, \overline{\boldsymbol{u}}, \overline{\boldsymbol{\lambda}}_{D}, \overline{\boldsymbol{\lambda}}_{\nu}, \overline{\boldsymbol{\lambda}}_{\tau}\right)=\mathbf{0}
$$


It is also obvious that the assumptions on $\left(\gamma^{\prime}, \boldsymbol{u}^{\prime}, \boldsymbol{\lambda}_{D}^{\prime}, \boldsymbol{\lambda}_{\nu}^{\prime}, \boldsymbol{\lambda}_{\tau}^{\prime}\right)$ guarantee that

$$
\begin{aligned}
\boldsymbol{\nabla} \boldsymbol{H}^{\left(i_{0}\right)}\left(\bar{\gamma}, \overline{\boldsymbol{u}}, \overline{\boldsymbol{\lambda}}_{D}, \overline{\boldsymbol{\lambda}}_{\nu}, \overline{\boldsymbol{\lambda}}_{\tau}\right)\left(\gamma^{\prime}, \boldsymbol{u}^{\prime}, \boldsymbol{\lambda}_{D}^{\prime}, \boldsymbol{\lambda}_{\nu}^{\prime}, \boldsymbol{\lambda}_{\tau}^{\prime}\right) \\
=\boldsymbol{H}^{\prime}\left(\left(\bar{\gamma}, \overline{\boldsymbol{u}}, \overline{\boldsymbol{\lambda}}_{D}, \overline{\boldsymbol{\lambda}}_{\nu}, \overline{\boldsymbol{\lambda}}_{\tau}\right) ;\left(\gamma^{\prime}, \boldsymbol{u}^{\prime}, \boldsymbol{\lambda}_{D}^{\prime}, \boldsymbol{\lambda}_{\nu}^{\prime}, \boldsymbol{\lambda}_{\tau}^{\prime}\right)\right)=\mathbf{0} .
\end{aligned}
$$

Next, if a selection function $\boldsymbol{H}^{(i)}$ corresponds to $\tilde{I}_{\mathrm{z}}^{\mathrm{f}}, \tilde{I}_{\mathrm{z}}^{\mathrm{c}}, \tilde{I}_{\mathrm{ci}}^{\mathrm{s}}, \tilde{I}_{\mathrm{ci}}^{\mathrm{l}}, \tilde{I}_{\mathrm{zi}}^{\mathrm{cs}}, \tilde{I}_{\mathrm{zi}}^{\mathrm{cl} \pm}$ forming decompositions of $I_{\mathrm{z}}, I_{\mathrm{c}} \cap I_{\mathrm{i}}$ and $\tilde{I}_{\mathrm{z}}^{\mathrm{c}} \cap I_{\mathrm{i}}$, from which at least one differs from the decompositions constituted by $I_{\mathrm{z}}^{\mathrm{f}}, I_{\mathrm{z}}^{\mathrm{c}}, I_{\mathrm{ci}}^{\mathrm{s}}, I_{\mathrm{ci}}^{\mathrm{l}}, I_{\mathrm{zi}}^{\mathrm{cs}}$ and $I_{\mathrm{zi}}^{\mathrm{cl} \pm}$, then we claim that

$$
\boldsymbol{\nabla} \boldsymbol{H}^{(i)}\left(\bar{\gamma}, \overline{\boldsymbol{u}}, \overline{\boldsymbol{\lambda}}_{D}, \overline{\boldsymbol{\lambda}}_{\nu}, \overline{\boldsymbol{\lambda}}_{\tau}\right)\left(\gamma^{\prime}, \boldsymbol{u}^{\prime}, \boldsymbol{\lambda}_{D}^{\prime}, \boldsymbol{\lambda}_{\nu}^{\prime}, \boldsymbol{\lambda}_{\tau}^{\prime}\right) \neq \mathbf{0}
$$

Indeed, making use of (51) and the assumption (i), one gets either

$$
j \in I_{\mathrm{z}}^{\mathrm{f}} \cap \tilde{I}_{\mathrm{z}}^{\mathrm{c}} \Longrightarrow-\left(\boldsymbol{B}_{\nu} \boldsymbol{u}^{\prime}\right)_{j}=\lambda_{\nu, j}^{\prime}-\left(\boldsymbol{B}_{\nu} \boldsymbol{u}^{\prime}\right)_{j}>0
$$

or

$$
j \in I_{\mathrm{z}}^{\mathrm{c}} \cap \tilde{I}_{\mathrm{z}}^{\mathrm{f}} \Longrightarrow \lambda_{\nu, j}^{\prime}=\lambda_{\nu, j}^{\prime}-\left(\boldsymbol{B}_{\nu} \boldsymbol{u}^{\prime}\right)_{j}<0
$$

if the decompositions of $I_{\mathrm{z}}^{\mathrm{c}}$ are not the same. In a similar way, assuming that the decompositions of $I_{z}$ are the same but at least one of the other decompositions does not coincide, one can show that

$$
\begin{aligned}
& j \in I_{\mathrm{ci}}^{\mathrm{s}} \cap \tilde{I}_{\mathrm{ci}}^{\mathrm{l}} \Longrightarrow-\tilde{s}_{j} \lambda_{\tau, j}^{\prime}+\mathscr{F}_{j}\left(\lambda_{\nu, j}^{\prime}-\left(\boldsymbol{B}_{\nu} \boldsymbol{u}^{\prime}\right)_{j}\right)<0, \\
& j \in\left(I_{\mathrm{ci}}^{\mathrm{l}} \cap \tilde{I}_{\mathrm{ci}}^{\mathrm{s}}\right) \cup\left(\left(I_{\mathrm{zi}}^{\mathrm{cl}+} \cup I_{\mathrm{zi}}^{\mathrm{cl}-}\right) \cap \tilde{I}_{\mathrm{zi}}^{\mathrm{cs}}\right) \Longrightarrow s_{j}\left(\left(\boldsymbol{C}_{\tau} \boldsymbol{u}^{\prime}\right)_{j}-\gamma^{\prime} V_{\tau, j}\right)>0, \\
& j \in\left(I_{\mathrm{zi}}^{\mathrm{cs}} \cap\left(\tilde{I}_{\mathrm{zi}}^{\mathrm{cl}+} \cup \tilde{I}_{\mathrm{zi}}^{\mathrm{cl}-}\right)\right) \cup\left(I_{\mathrm{zi}}^{\mathrm{cl}+} \cap \tilde{I}_{\mathrm{zi}}^{\mathrm{cl}-}\right) \cup\left(I_{\mathrm{zi}}^{\mathrm{cl}-} \cap \tilde{I}_{\mathrm{zi}}^{\mathrm{cl}+}\right) \\
& \Longrightarrow \lambda_{\tau, j}^{\prime}-\tilde{s}_{j} \mathscr{F}_{j}\left(\lambda_{\nu, j}^{\prime}-\left(\boldsymbol{B}_{\nu} \boldsymbol{u}^{\prime}\right)_{j}\right) \neq 0,
\end{aligned}
$$

where $s_{j}$ are defined by $(46)$ and

$$
\tilde{s}_{j}= \begin{cases}-\operatorname{sgn}\left(\bar{\lambda}_{\tau, j}\right) & \text { if } j \in \tilde{I}_{\mathrm{ci}}^{\mathrm{l}} \\ \pm 1 & \text { if } j \in \tilde{I}_{\mathrm{zi}}^{\mathrm{cl}}\end{cases}
$$

As $\left(6^{\prime}\right)$ (iii) is fulfilled directly by the assumption (ii), existence of the announced curve $\boldsymbol{c}$ follows from the first part of Theorem 2 and Remark 2 .

Now, let $\tilde{\boldsymbol{c}}$ be a curve from the second part of the present assertion. Comparing (5) and $(50)$, we have to show $(5)(\widetilde{\mathrm{jjj}})$ :

$$
\tilde{\boldsymbol{c}}^{\prime}(\bar{s}) \in \bigcup_{r>0} r\left(\gamma^{\prime}, \boldsymbol{u}^{\prime}, \boldsymbol{\lambda}_{D}^{\prime}, \boldsymbol{\lambda}_{\nu}^{\prime}, \boldsymbol{\lambda}_{\tau}^{\prime}\right)
$$

One can see from $(50)(\widetilde{\mathrm{jj}})$ that $(\boldsymbol{H} \circ \tilde{\boldsymbol{c}})^{\prime}(\bar{s})=\mathbf{0}$. In addition, one can deduce from Proposition 2 that $(\boldsymbol{H} \circ \tilde{\boldsymbol{c}})^{\prime}(\bar{s})=\boldsymbol{H}^{\prime}\left(\tilde{\boldsymbol{c}}(\bar{s}) ; \tilde{\boldsymbol{c}}^{\prime}(\bar{s})\right)$. Taking into account $(50)(\widetilde{\mathrm{j}})$ and $(\widetilde{\mathrm{jjj}})$, we thus have in a similar way as before that

$$
\boldsymbol{\nabla} \boldsymbol{H}^{\left(i_{0}\right)}\left(\bar{\gamma}, \overline{\boldsymbol{u}}, \overline{\boldsymbol{\lambda}}_{D}, \overline{\boldsymbol{\lambda}}_{\nu}, \overline{\boldsymbol{\lambda}}_{\tau}\right) \tilde{\boldsymbol{c}}^{\prime}(\bar{s})=\boldsymbol{H}^{\prime}\left(\tilde{\boldsymbol{c}}(\bar{s}) ; \tilde{\boldsymbol{c}}^{\prime}(\bar{s})\right)=\mathbf{0}
$$


This combined with (51) and the assumptions (i) and (ii) implies that

$$
\tilde{\boldsymbol{c}}^{\prime}(\bar{s}) \in \operatorname{span}\left\{\left(\gamma^{\prime}, \boldsymbol{u}^{\prime}, \boldsymbol{\lambda}_{D}^{\prime}, \boldsymbol{\lambda}_{\nu}^{\prime}, \boldsymbol{\lambda}_{\tau}^{\prime}\right)\right\} .
$$

But $\left(\gamma^{\prime}, \boldsymbol{u}^{\prime}, \boldsymbol{\lambda}_{D}^{\prime}, \boldsymbol{\lambda}_{\nu}^{\prime}, \boldsymbol{\lambda}_{\tau}^{\prime}\right)$ satisfies all the inequalities in (49) strictly, so it is clear that these conditions are violated for any $r\left(\gamma^{\prime}, \boldsymbol{u}^{\prime}, \boldsymbol{\lambda}_{D}^{\prime}, \boldsymbol{\lambda}_{\nu}^{\prime}, \boldsymbol{\lambda}_{\tau}^{\prime}\right)$ with $r<0$ and any $j \in I_{\mathrm{z}}^{\mathrm{f}} \cup I_{\mathrm{z}}^{\mathrm{c}} \cup I_{\mathrm{ci}}^{\mathrm{s}} \cup$ $I_{\mathrm{ci}}^{\mathrm{l}} \cup I_{\mathrm{zi}}^{\mathrm{cs}} \cup I_{\mathrm{zi}}^{\mathrm{cl} \pm}=I_{\mathrm{z}} \cup\left(I_{\mathrm{c}} \cap I_{\mathrm{i}}\right)$, which is assumed to be non-empty. This together with $(50)(\widetilde{\mathrm{jjj}})$ leads to $(5)(\widetilde{\mathrm{jjj}})$ and the uniqueness part of Theorem 2 applies.

In the trivial case when $I_{\mathrm{z}} \cup\left(I_{\mathrm{c}} \cap I_{\mathrm{i}}\right)=\emptyset, \boldsymbol{H}$ is smooth in some vicinity of $\left(\bar{\gamma}, \overline{\boldsymbol{u}}, \overline{\boldsymbol{\lambda}}_{D}, \overline{\boldsymbol{\lambda}}_{\nu}, \overline{\boldsymbol{\lambda}}_{\tau}\right)$ and the classical implicit function theorem can be employed.

Theorem 4. Let Assumption 1 hold, $\left(\bar{\gamma}, \overline{\boldsymbol{u}}, \overline{\boldsymbol{\lambda}}_{D}, \overline{\boldsymbol{\lambda}}_{\nu}, \overline{\boldsymbol{\lambda}}_{\tau}\right)$ be a solution of $(\mathcal{P})$ with $I_{\mathrm{z}} \cup\left(I_{\mathrm{c}} \cap\right.$ $\left.I_{\mathrm{i}}\right)=\emptyset$ and $\left(\gamma^{\prime}, \boldsymbol{u}^{\prime}, \boldsymbol{\lambda}_{D}^{\prime}, \boldsymbol{\lambda}_{\nu}^{\prime}, \boldsymbol{\lambda}_{\tau}^{\prime}\right)$ be a non-zero vector solving the first-order system $\left(\mathcal{P}^{\prime} 1\right)$, $\left(\mathcal{P}^{\prime} 2\right)$, $\left(\mathcal{P}^{\prime} 3^{\prime}\right)$ and $\left(\mathcal{P}^{\prime} 4^{\prime}\right)$. If $\boldsymbol{\nabla} \boldsymbol{H}\left(\bar{\gamma}, \overline{\boldsymbol{u}}, \overline{\boldsymbol{\lambda}}_{D}, \overline{\boldsymbol{\lambda}}_{\nu}, \overline{\boldsymbol{\lambda}}_{\tau}\right)$ has the maximal rank, then there exist $\delta>0$ and a $C^{1}$-curve $\boldsymbol{c}:(\bar{s}-\delta, \bar{s}+\delta) \rightarrow \mathbb{R}^{1+2\left(n_{\Omega}+n_{D}+n_{c}\right)}$ such that

$\boldsymbol{c}(\bar{s})=\left(\bar{\gamma}, \overline{\boldsymbol{u}}, \overline{\boldsymbol{\lambda}}_{D}, \overline{\boldsymbol{\lambda}}_{\nu}, \overline{\boldsymbol{\lambda}}_{\tau}\right), \quad \boldsymbol{H}(\boldsymbol{c}(s))=\mathbf{0}, \forall s \in(\bar{s}-\delta, \bar{s}+\delta), \quad \boldsymbol{c}^{\prime}(\bar{s})=\left(\gamma^{\prime}, \boldsymbol{u}^{\prime}, \boldsymbol{\lambda}_{D}^{\prime}, \boldsymbol{\lambda}_{\nu}^{\prime}, \boldsymbol{\lambda}_{\tau}^{\prime}\right)$.

Moreover, the image of any other solution curve passing through $\left(\bar{\gamma}, \overline{\boldsymbol{u}}, \overline{\boldsymbol{\lambda}}_{D}, \overline{\boldsymbol{\lambda}}_{\nu}, \overline{\boldsymbol{\lambda}}_{\tau}\right)$ is in some neighbourhood of $\left(\bar{\gamma}, \overline{\boldsymbol{u}}, \overline{\boldsymbol{\lambda}}_{D}, \overline{\boldsymbol{\lambda}}_{\nu}, \overline{\boldsymbol{\lambda}}_{\tau}\right)$ contained in the image of $\boldsymbol{c}$.

Remark 6. Assume that $\boldsymbol{H}^{\prime}\left(\left(\bar{\gamma}, \overline{\boldsymbol{u}}, \overline{\boldsymbol{\lambda}}_{D}, \overline{\boldsymbol{\lambda}}_{\nu}, \overline{\boldsymbol{\lambda}}_{\tau}\right) ;\left(\gamma^{\prime}, \boldsymbol{u}^{\prime}, \boldsymbol{\lambda}_{D}^{\prime}, \boldsymbol{\lambda}_{\nu}^{\prime}, \boldsymbol{\lambda}_{\tau}^{\prime}\right)\right)=\mathbf{0}$. Then one can easily verify that (49) holds strictly if and only if

$$
\left.\begin{array}{rll}
\lambda_{\nu, j}^{\prime}=0,\left(\boldsymbol{B}_{\nu} \boldsymbol{u}^{\prime}\right)_{j}<0, & j \in I_{\mathrm{z}}^{\mathrm{f}} & (\rightarrow \text { separation }) ; \\
\left(\boldsymbol{B}_{\nu} \boldsymbol{u}^{\prime}\right)_{j}=0, \lambda_{\nu, j}^{\prime}<0, & j \in I_{\mathrm{z}}^{\mathrm{c}} & (\rightarrow \text { strong contact }) ; \\
\left(\boldsymbol{C}_{\tau} \boldsymbol{u}^{\prime}\right)_{j}-\gamma^{\prime} V_{\tau, j}=0,-s_{j} \lambda_{\tau, j}^{\prime}<-\mathscr{F}_{j} \lambda_{\nu, j}^{\prime}, & j \in I_{\mathrm{ci}}^{\mathrm{s}} & (\rightarrow \text { strong stick }) ; \\
\left(\boldsymbol{C}_{\tau} \boldsymbol{u}^{\prime}\right)_{j}-\gamma^{\prime} V_{\tau, j}=0,\left|\lambda_{\tau, j}^{\prime}\right|<-\mathscr{F}_{j} \lambda_{\nu, j}^{\prime}, & j \in I_{\mathrm{zi}}^{\mathrm{cs}} & (\rightarrow \text { strong stick }) ; \\
\lambda_{\tau, j}^{\prime}-s_{j} \mathscr{F}_{j} \lambda_{\nu, j}^{\prime}=0, s_{j}\left(\left(\boldsymbol{C}_{\tau} \boldsymbol{u}^{\prime}\right)_{j}-\gamma^{\prime} V_{\tau, j}\right)>0, & j \in I_{\mathrm{ci}}^{1} \cup I_{\mathrm{zi}}^{\mathrm{cl}+} \cup I_{\mathrm{zi}}^{\mathrm{cl}-} \\
& (\rightarrow \text { non-zero slip })
\end{array}\right\}
$$

(compare to $\left(\mathcal{P}^{\prime} 3^{\prime}\right)$ and $\left.\left(\mathcal{P}^{\prime} 4^{\prime}\right)\right)$. This means that the direction $\left(\gamma^{\prime}, \boldsymbol{u}^{\prime}, \boldsymbol{\lambda}_{D}^{\prime}, \boldsymbol{\lambda}_{\nu}^{\prime}, \boldsymbol{\lambda}_{\tau}^{\prime}\right)$ satisfying the assumption (i) in Theorem 3 leads to solutions with strict contact modes of all nodes, without any grazing contact or impending slip.

In a similar way, non-strict inequalities in (49) correspond to (52) with non-strict inequalities and non-strict nodal contact modes. Thus, the uniqueness part of Theorem 3 means that there is only one branch of solutions with non-strict contact modes corresponding to (49). From this mechanical point of view, the claim of Theorem 3 resembles the one of [22, Proposition 8].

Proposition 9 (Satisfaction of the assumption (ii) in Theorem 3). Let $(\mathcal{P})$ correspond to a model of small-deformation elasticity with a body properly constrained by the Dirichlet condition. If $g$ satisfies Assumption 1 and $\left\|\nabla^{2} g\right\| /\|\nabla g\|$ and $\mathscr{F}_{j}, j \in\left(I_{\mathrm{c}} \cup I_{\mathrm{z}}\right) \cap\left(I_{1} \cup I_{\mathrm{i}}\right)$ are small, then the gradients of all selection functions $\boldsymbol{H}^{(i)}$ given by (48) have the maximal rank for any $\boldsymbol{P} \in \mathbb{R}^{2 n_{\Omega}}$ and any $\boldsymbol{U}_{D}^{\prime} \in \mathbb{R}^{2 n_{D}}$. 
Proof. We shall argue similarly as in [5, proof of Proposition 3.2]. By (48),

$$
\boldsymbol{\nabla} \boldsymbol{H}^{(i)}\left(\bar{\gamma}, \overline{\boldsymbol{u}}, \overline{\boldsymbol{\lambda}}_{D}, \overline{\boldsymbol{\lambda}}_{\nu}, \overline{\boldsymbol{\lambda}}_{\tau}\right)=\left(\begin{array}{ccccc}
-\boldsymbol{P} & \boldsymbol{K} & -\boldsymbol{B}_{D}^{\top} & -\boldsymbol{B}_{\nu}^{\top} & -\boldsymbol{B}_{\tau}^{\top} \\
-\boldsymbol{U}_{D}^{\prime} & \boldsymbol{B}_{D} & \mathbf{0} & \mathbf{0} & \mathbf{0} \\
\mathbf{0} & \mathbf{0} & \mathbf{0} & \boldsymbol{I}_{\mathrm{F}, I} & \mathbf{0} \\
\mathbf{0} & \left(\boldsymbol{B}_{\nu}\right)_{\mathrm{C}, J} & \mathbf{0} & \mathbf{0} & \mathbf{0} \\
\mathbf{0} & \mathbf{0} & \mathbf{0} & \mathbf{0} & \boldsymbol{I}_{\mathrm{N}, I} \\
-\left(\boldsymbol{V}_{\tau}\right)_{\mathrm{S}} & \left(\boldsymbol{C}_{\tau}\right)_{\mathrm{S}, J} & \mathbf{0} & \mathbf{0} & \mathbf{0} \\
\mathbf{0} & \left(\boldsymbol{S} \boldsymbol{F} \boldsymbol{B}_{\nu}\right)_{\mathrm{L}, J} & \mathbf{0} & -(\boldsymbol{S} \boldsymbol{F})_{\mathrm{L}, I} & \boldsymbol{I}_{\mathrm{L}, I}
\end{array}\right),
$$

where $J=\left\{1, \ldots, 2 n_{\Omega}\right\}, \mathrm{F}$ denotes jointly the indices from $I_{\mathrm{f}}$ and $I_{\mathrm{z}}^{\mathrm{f}}, \mathrm{C}$ the indices from $I_{\mathrm{c}}$ and $I_{\mathrm{z}}^{\mathrm{c}}, \mathrm{N}$ the indices from $I_{\mathrm{f}}, I_{\mathrm{z}}^{\mathrm{f}}$ and $I_{0}, \mathrm{~S}$ the indices from $I_{\mathrm{s}}, I_{\mathrm{ci}}^{\mathrm{s}}$ and $I_{\mathrm{zi}}^{\mathrm{cs}}$, and $\mathrm{L}$ the indices from $\left(I_{\mathrm{c}} \cap I_{1}\right), I_{\mathrm{zl}}^{\mathrm{c}}, I_{\mathrm{ci}}^{\mathrm{l}}$ and $I_{\mathrm{zi}}^{\mathrm{cl} \pm}$. Further,

$$
\begin{aligned}
& \boldsymbol{S}=\operatorname{Diag}\left(s_{11}, \ldots, s_{n_{c} n_{c}}\right), \quad s_{j j}= \begin{cases}s_{j} & \text { if } j \in\left(I_{\mathrm{c}} \cap I_{1}\right) \cup I_{\mathrm{zl}}^{\mathrm{c}} \cup I_{\mathrm{ci}}^{1} \cup I_{\mathrm{zi}}^{\mathrm{cl}}, \\
0 & \text { otherwise }\end{cases} \\
& \boldsymbol{F}=\operatorname{Diag}\left(\mathscr{F}_{1}, \ldots, \mathscr{F}_{n_{c}}\right) .
\end{aligned}
$$

Suppose first that $\nabla^{2} g=\mathbf{0}$ and $\mathscr{F}_{j}=0$ for any $j \in\left(I_{\mathrm{c}} \cup I_{\mathrm{z}}\right) \cap\left(I_{1} \cup I_{\mathrm{i}}\right)$. Then $\boldsymbol{K}$ is positive definite on $\operatorname{Ker}\left(\boldsymbol{B}_{D}\right), \boldsymbol{C}_{\tau}=\boldsymbol{B}_{\tau}, \boldsymbol{S} \boldsymbol{F}=\mathbf{0}$ and we claim that the partial gradient $\boldsymbol{\nabla}_{\left(\boldsymbol{u}, \boldsymbol{\lambda}_{D}, \boldsymbol{\lambda}_{\nu}, \boldsymbol{\lambda}_{\tau}\right)} \boldsymbol{H}^{(i)}\left(\bar{\gamma}, \overline{\boldsymbol{u}}, \overline{\boldsymbol{\lambda}}_{D}, \overline{\boldsymbol{\lambda}}_{\nu}, \overline{\boldsymbol{\lambda}}_{\tau}\right)$ is non-singular for any $\boldsymbol{H}^{(i)}$. Indeed, one has

$$
\nabla_{\left(\boldsymbol{u}, \boldsymbol{\lambda}_{D}, \boldsymbol{\lambda}_{\nu}, \boldsymbol{\lambda}_{\tau}\right)} \boldsymbol{H}^{(i)}\left(\bar{\gamma}, \overline{\boldsymbol{u}}, \overline{\boldsymbol{\lambda}}_{D}, \overline{\boldsymbol{\lambda}}_{\nu}, \overline{\boldsymbol{\lambda}}_{\tau}\right)=\left(\begin{array}{cccc}
\boldsymbol{K} & -\boldsymbol{B}_{D}^{\top} & -\boldsymbol{B}_{\nu}^{\top} & -\boldsymbol{B}_{\tau}^{\top} \\
\boldsymbol{B}_{D} & \mathbf{0} & \mathbf{0} & \mathbf{0} \\
\mathbf{0} & \mathbf{0} & \boldsymbol{I}_{\mathrm{F}, I} & \mathbf{0} \\
\left(\boldsymbol{B}_{\nu}\right)_{\mathrm{C}, J} & \mathbf{0} & \mathbf{0} & \mathbf{0} \\
\mathbf{0} & \mathbf{0} & \mathbf{0} & \boldsymbol{I}_{\mathrm{N}, I} \\
\left(\boldsymbol{B}_{\tau}\right)_{\mathrm{S}, J} & \mathbf{0} & \mathbf{0} & \mathbf{0} \\
\mathbf{0} & \mathbf{0} & \mathbf{0} & \boldsymbol{I}_{\mathrm{L}, I}
\end{array}\right),
$$

which is obviously a non-singular matrix iff the matrix

$$
\boldsymbol{J}:=\left(\begin{array}{cccc}
\boldsymbol{K} & -\boldsymbol{B}_{D}^{\top} & -\left(\boldsymbol{B}_{\nu}^{\top}\right)_{J, \mathrm{C}} & -\left(\boldsymbol{B}_{\tau}^{\top}\right)_{J, \mathrm{~S}} \\
\boldsymbol{B}_{D} & \mathbf{0} & \mathbf{0} & \mathbf{0} \\
\left(\boldsymbol{B}_{\nu}\right)_{\mathrm{C}, J} & \mathbf{0} & \mathbf{0} & \mathbf{0} \\
\left(\boldsymbol{B}_{\tau}\right)_{\mathrm{S}, J} & \mathbf{0} & \mathbf{0} & \mathbf{0}
\end{array}\right),
$$

is non-singular. But taking any $\left(\boldsymbol{u}^{\prime}, \boldsymbol{\lambda}_{D}^{\prime}, \boldsymbol{\lambda}_{\nu \mathrm{C}}^{\prime}, \boldsymbol{\lambda}_{\tau \mathrm{S}}^{\prime}\right)$ such that $\boldsymbol{J}\left(\boldsymbol{u}^{\prime}, \boldsymbol{\lambda}_{D}^{\prime}, \boldsymbol{\lambda}_{\nu \mathrm{C}}^{\prime}, \boldsymbol{\lambda}_{\tau \mathrm{S}}^{\prime}\right)=\mathbf{0}$, one gets from the last three lines of blocks that

$$
\boldsymbol{u}^{\prime} \in\left(\operatorname{Ker}\left(\boldsymbol{B}_{D}\right) \cap \operatorname{Ker}\left(\left(\boldsymbol{B}_{\nu}\right)_{\mathrm{C}, J}\right) \cap \operatorname{Ker}\left(\left(\boldsymbol{B}_{\tau}\right)_{\mathrm{S}, J}\right)\right) \subset \operatorname{Ker}\left(\boldsymbol{B}_{D}\right) .
$$

This together with the first line of blocks of the system gives

$$
0=\boldsymbol{u}^{\prime \top}\left(\boldsymbol{K} \boldsymbol{u}^{\prime}-\boldsymbol{B}_{D}^{\top} \boldsymbol{\lambda}_{D}^{\prime}-\left(\boldsymbol{B}_{\nu}^{\top}\right)_{J, \mathrm{C}} \boldsymbol{\lambda}_{\nu \mathrm{C}}^{\prime}-\left(\boldsymbol{B}_{\tau}^{\top}\right)_{J, \mathrm{~S}} \boldsymbol{\lambda}_{\tau \mathrm{S}}^{\prime}\right)=\boldsymbol{u}^{\prime \top} \boldsymbol{K} \boldsymbol{u}^{\prime}
$$


and positive definiteness of $\boldsymbol{K}$ on $\operatorname{Ker}\left(\boldsymbol{B}_{D}\right)$ implies that $\boldsymbol{u}^{\prime}=\mathbf{0}$. Consequently,

$$
\boldsymbol{B}_{D}^{\top} \boldsymbol{\lambda}_{D}^{\prime}+\left(\boldsymbol{B}_{\nu}^{\top}\right)_{J, \mathrm{C}} \boldsymbol{\lambda}_{\nu \mathrm{C}}^{\prime}+\left(\boldsymbol{B}_{\tau}^{\top}\right)_{J, \mathrm{~S}} \boldsymbol{\lambda}_{\tau \mathrm{S}}^{\prime}=\mathbf{0}
$$

and taking into account Remark 3 , we have necessarily $\boldsymbol{\lambda}_{D}^{\prime}=\mathbf{0}, \boldsymbol{\lambda}_{\nu \mathrm{C}}^{\prime}=\mathbf{0}, \boldsymbol{\lambda}_{\tau \mathrm{S}}^{\prime}=\mathbf{0}$ due to full row rank of $\left(\begin{array}{c}\boldsymbol{B}_{D} \\ \boldsymbol{B}_{\nu} \\ \boldsymbol{B}_{\tau}\end{array}\right)$. This shows that $\boldsymbol{J}$ is non-singular and the conclusion is valid in the special case.

Continuous dependence on the elements of $\nabla^{2} g /\|\nabla g\|$ and $\mathscr{F}_{j}$ completes the assertion.

\subsection{Numerical Experiments}

This subsection presents results computed with the predictor-corrector continuation method described in Subsection 2.2 and performed with the finite-element library GetFEM++ [25].

\subsubsection{V-Shaped Body}

The first experiment is done for a V-shaped body from [22, Subsection 8.2], which is depicted in Figure 10(a). It is approximately $0.82 \mathrm{~m}$ wide, $0.8 \mathrm{~m}$ high and we consider a plane-strain model of small-deformation elasticity with the Lamé constants $\lambda=$ $100 \mathrm{GN} / \mathrm{m}^{2}$ and $\mu=82 \mathrm{GN} / \mathrm{m}^{2}$. The body is subject to volume forces of the density $\boldsymbol{f}(\gamma)=\left(-5 \mathrm{GN} / \mathrm{m}^{3}, 1-9 \gamma \mathrm{GN} / \mathrm{m}^{3}\right)$ and it is fixed along both parts of $\Gamma_{D}$ and free of surface tractions on $\Gamma_{N}$, that is, $\boldsymbol{U}_{D}=\mathbf{0}$ and $\boldsymbol{h}=\mathbf{0}$. The continuation problem is taken with $\boldsymbol{w}=\mathbf{0}$ and $\boldsymbol{V}_{\tau}=\mathbf{0}$.

Three unstructured meshes with 2220, 8880 and 35520 linear triangles (M2220, M8880 and M35520) were used for the finite-element discretisation. The numerical continuation was initialised with $\varepsilon=\varepsilon^{\prime}=5 \cdot 10^{-12}, c_{\min }=0.99999, j_{\mathrm{thr}}=4, j_{\max }=5, h_{\mathrm{dec}}=0.5$, $h_{\mathrm{inc}}=1.3, h_{\min }=5 \cdot 10^{-7}, h_{\max }=0.1$. When the predictor-corrector failed, Algorithm 2 was run with $h_{1}=1.5 h_{\min }, h_{2}=h_{\min }$ and $c_{\min }=0.99999$. The values of $h_{2}$ were increased by adding $h_{\min } \cdot 10^{\left\lfloor\log _{10}\left(h / h_{\text {min }}\right)\right\rfloor}$ to it in the case of performing the last line of Step 3 of Algorithm 2, where $\lfloor$.$\rfloor stands for the lower integer part. After a change of the tangent,$ the predictor-corrector was restarted with $h=5 \cdot 10^{-4}$.

Solution curves for the three meshes and the friction coefficient $\mathscr{F}$ chosen to be 1.8 are visualised by displacements and contact stresses at the most left contact node, that is, in the lower left corner of the body, in Figure 9. There is a non-vanishing gap between the body and the obstacle for $\gamma=0$ for all meshes. Whereas the displacements are almost the same for all solution curves, the contact stresses do not seem to converge at this corner node. Nevertheless, one can observe that the qualitative features of all the curves remain the same: they all possess two non-smooth folds for approximately the same values of the parameter $\gamma$, which means that there are up to three different solutions of the contact problem for a fixed value of $\gamma$.

Deformed bodies corresponding to three different solutions for $\gamma=0.38$ are depicted in Figures 10(b)-(d) (the displacements are ten times amplified): the body is separated from 

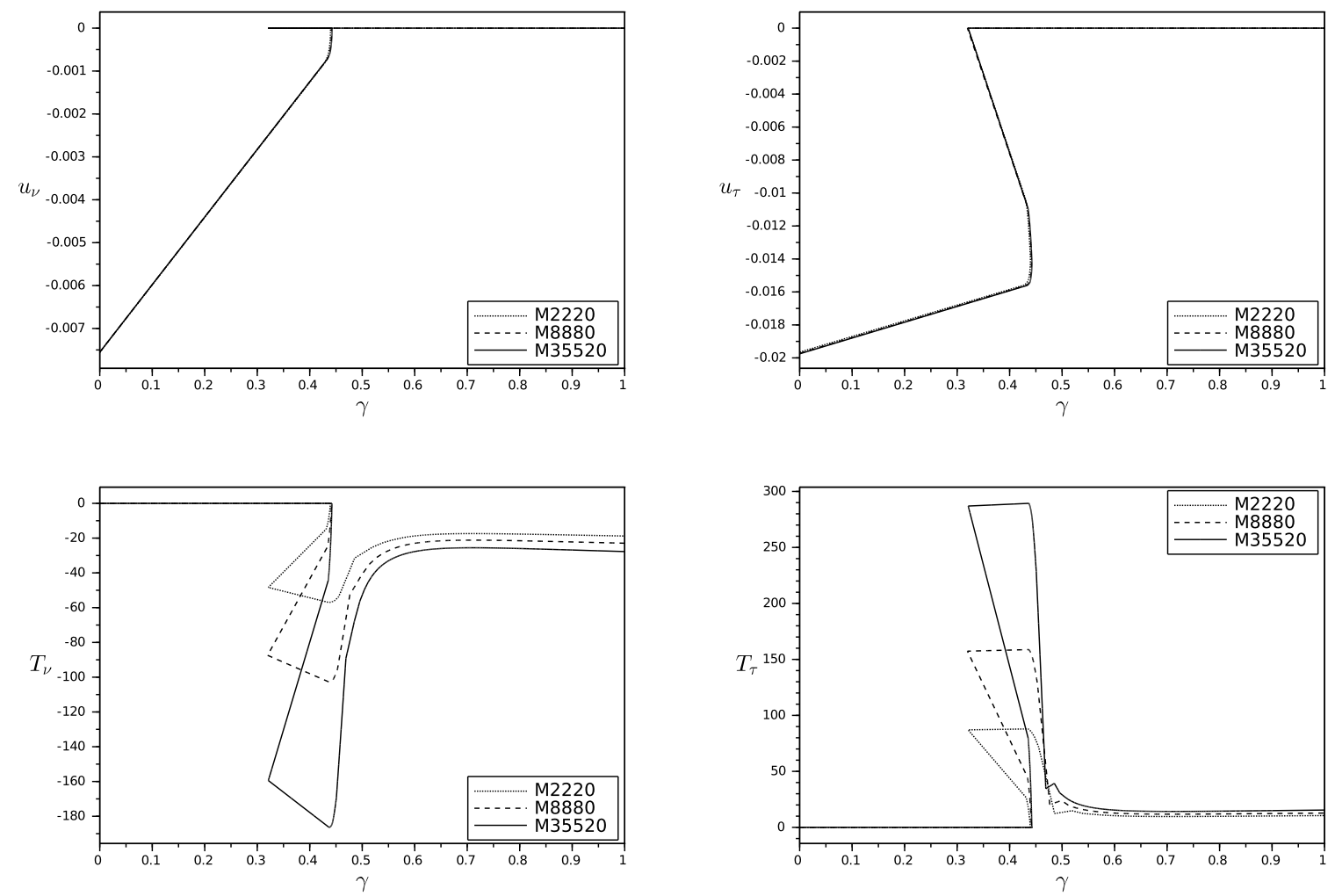

Figure 9: The normal and tangential displacements $u_{\nu}$ and $u_{\tau}$ and the normal and tangential contact stresses $T_{\nu}$ and $T_{\tau}$ at the most left contact node for $\mathscr{F}=1.8$.

the foundation in (b), and the most left contact node is in left slip and in stick in (c) and (d), respectively.

Figure 11 compares solution curves for various values of the friction coefficient $\mathscr{F}$. It gives us an idea how solution curves behave when $\mathscr{F}$ increases from sufficiently small values guaranteeing existence and uniqueness of solutions of first-order systems to values with possible non-existence or non-uniqueness of the solutions.

\subsubsection{Rectangular Block}

In the second experiment, we model contact of a rectangular block that is $40 \mathrm{~mm}$ wide and $80 \mathrm{~mm}$ high with a flat rigid foundation similarly as in [23] (see Figure 13(a) for the geometry). A plane-strain approximation of the nonlinear Ciarlet-Geymonat constitutive law described in [7, Chapter 4] is considered:

$$
\begin{gathered}
\hat{\boldsymbol{\sigma}}(\boldsymbol{x}, \boldsymbol{F})=(\tilde{\boldsymbol{\sigma}}(\tilde{\boldsymbol{F}}))_{1 \leq i, j \leq 2}, \quad \tilde{\boldsymbol{F}}=\left(\begin{array}{cc}
\boldsymbol{F} & \mathbf{0} \\
\mathbf{0} & 1
\end{array}\right), \quad \boldsymbol{F} \in \mathbb{R}^{2 \times 2}, \\
\tilde{\boldsymbol{\sigma}}(\tilde{\boldsymbol{F}})=2 b\left(\operatorname{tr}\left(\tilde{\boldsymbol{F}}^{\top} \tilde{\boldsymbol{F}}\right)\right) \boldsymbol{I}+2\left(a-b \tilde{\boldsymbol{F}} \tilde{\boldsymbol{F}}^{\top}\right) \tilde{\boldsymbol{F}}+\left(2 c \operatorname{det}\left(\tilde{\boldsymbol{F}}^{\top} \tilde{\boldsymbol{F}}\right)-d\right) \tilde{\boldsymbol{F}}^{-\top}, \quad \tilde{\boldsymbol{F}} \in \mathbb{R}^{3 \times 3},
\end{gathered}
$$




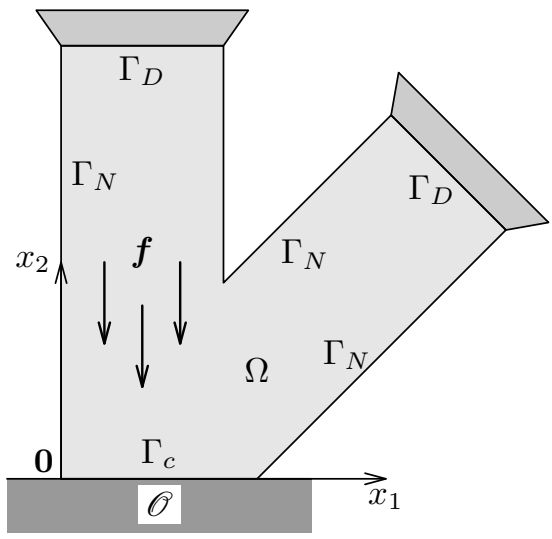

(a)

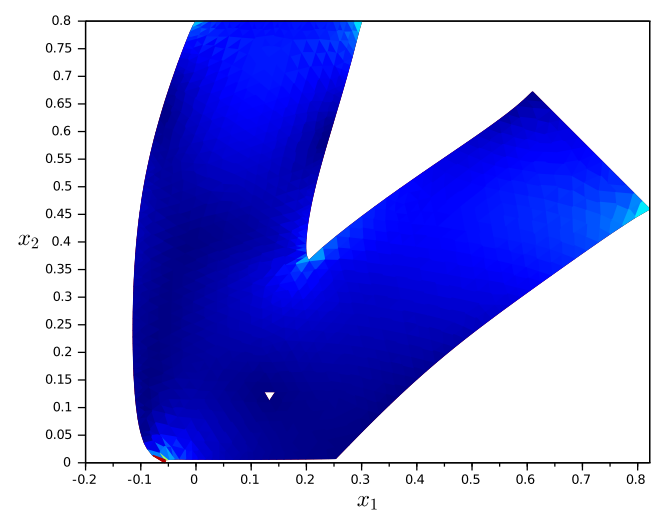

(c)

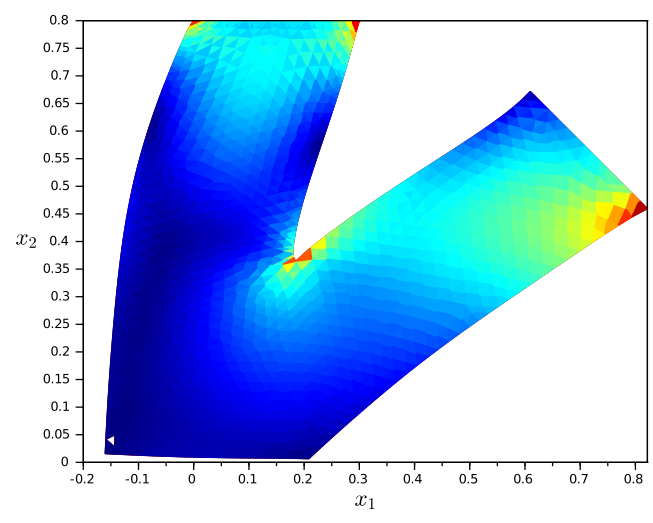

(b)

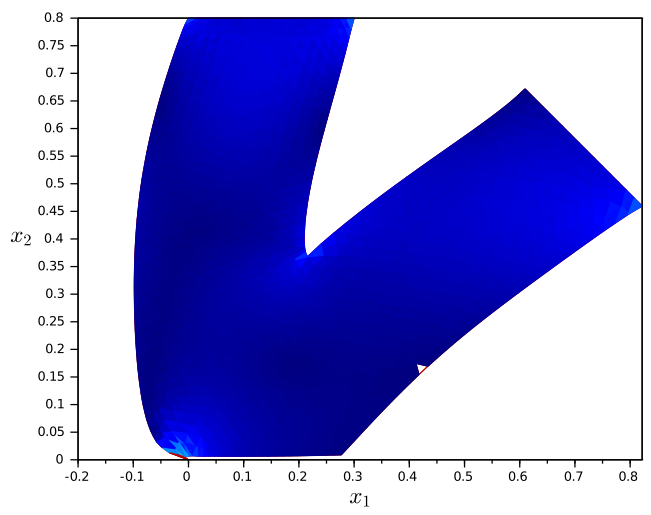

(d)

Figure 10: V-shaped body in contact with a rigid foundation: (a) reference configuration; (b) - (d) three solutions for $\gamma=0.38$ with $\mathscr{F}=1.8$.

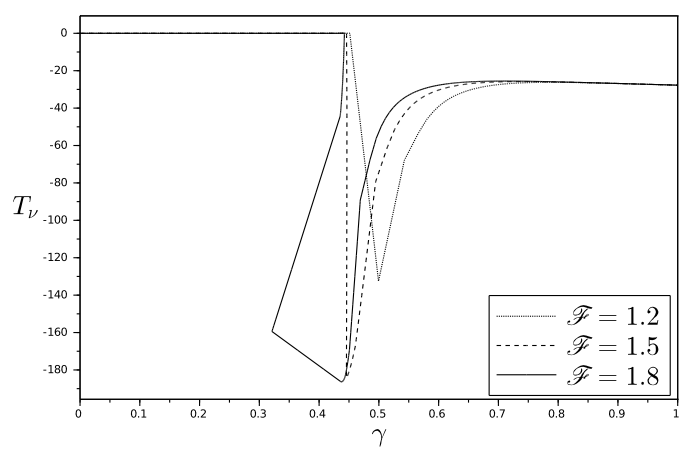

Figure 11: The normal contact stress at the most left contact node for various values of the friction coefficient. 
with

$$
\lambda=4000 \mathrm{~N} / \mathrm{mm}^{2}, \quad \mu=120 \mathrm{~N} / \mathrm{mm}^{2}, \quad a=30 \mathrm{~N} / \mathrm{mm}^{2}
$$

and

$$
b=\frac{\mu}{2}-a, \quad c=\frac{\lambda}{4}-\frac{\mu}{2}+a, \quad d=\frac{\lambda}{2}+\mu .
$$

External forces are neglected while the displacement

$$
\boldsymbol{U}_{D}(\gamma, \boldsymbol{x})=x_{1}\left(\begin{array}{c}
\cos 0.023-1 \\
\sin 0.023
\end{array}\right)+\gamma\left(\begin{array}{c}
0 \\
-1
\end{array}\right)
$$

is prescribed on the upper side of the block. Besides, $\boldsymbol{w}=\mathbf{0}$ and $\boldsymbol{V}_{\tau}=\mathbf{0}$.

The body is discretised with three uniform meshes with 800, 3200 and 12800 bilinear squares (M800, M3200 and M12800), respectively. The parameters for Algorithm 1 were $\varepsilon=\varepsilon^{\prime}=5 \cdot 10^{-10}, c_{\min }=0.99999, j_{\mathrm{thr}}=4, j_{\max }=5, h_{\mathrm{dec}}=0.35, h_{\mathrm{inc}}=1.3, h_{\min }=5 \cdot 10^{-7}$, $h_{\max }=0.5$, whereas the setting of Algorithm 2 was the same as in the previous experiment. The predictor-corrector was restarted with $h=5 \cdot 10^{-4}$.

Courses of solution curves for the three meshes and $\mathscr{F}=1.8$ are illustrated in Figure 12 by displacements and contact stresses at a contact node in the relative interior of the contact zone. Despite a bit oscillatory behaviour, contact stresses seem to converge along with displacements here. Again, there are two folds on the solution curves and up to three different solutions for a fixed $\gamma$. Figures 13(b)-(d) show a zoom near the contact zone of deformed bodies corresponding to three different solutions for $\gamma=0.495$ (the displacements are ten times amplified): the most right contact node is separated, in right slip and in stick in (b), (c) and (d), respectively. Formation of the non-smooth folds for increasing values of $\mathscr{F}$ can be observed in Figure 14.

\section{Conclusion}

We have proposed a continuation problem for finding solutions of discretised abstract firstorder evolution problems. The merit of the problem is that any solution from one time level of the discretised problem furnishes us with an initial point for computing solutions on the next time level. Confining ourselves to a $P C^{1}$ continuation problem, we have proved local existence and uniqueness of solution curves under assumptions required by an appropriate implicit function theorem. Moreover, we have derived a first-order system characterising one-sided tangents to curves solving the continuation problem and we have stated criteria guaranteeing existence and uniqueness of solutions of this system.

Possibility of continuation in the direction of a null vector of the first-order system has been deeply discussed. In particular, it has been shown that if the null vector lies in the kernel of the gradient of exactly one selection function and the gradient has maximal rank, then there exists a unique solution curve emanating in that direction (Theorem 2). In addition, it has been demonstrated on simple examples what can happen when one of these two assumptions is violated. We believe that this analysis gives insight into possible scenarios during piecewise-smooth continuation of general systems. 

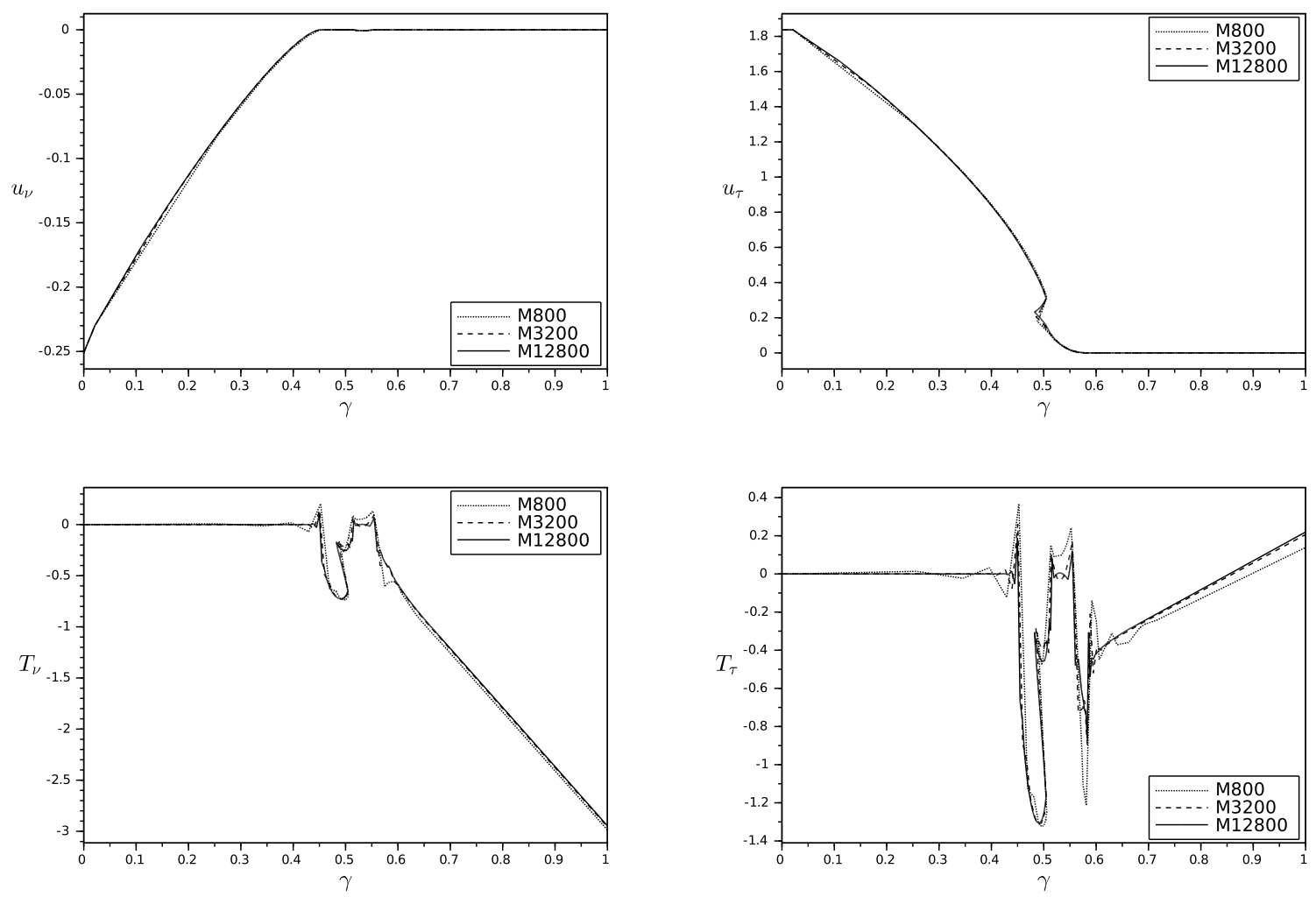

Figure 12: The normal and tangential displacements and the normal and tangential contact stresses at the node with the coordinates $(10 \mathrm{~mm}, 0 \mathrm{~mm})$ in the reference configuration for $\mathscr{F}=1.8$.

An application of the abstract continuation problem has been proposed for quasi-static plane contact problems with Coulomb friction. Its advantage over the rate problem as well as the merit of the initial velocity included in it have been illustrated on a simple example. Various formulations of the first-order system have been derived.

Results of existence and uniqueness of solutions of the first-order system from the abstract frame have been supplemented. In particular, it has been shown that nodes in strong contact with non-zero or impending slip and nodes in grazing contact with non-zero slip may be responsible for non-existence of solutions whereas nodes in strong or grazing contact with non-zero or impending slip may be responsible for multiple solutions.

Possibility of continuation in a direction solving the first-order system has been proved under the assumption that the direction leads to solutions with strict contact modes of all nodes and the gradient of the selection function corresponding to these modes has maximal rank. In addition, the continuation has been showed to be unique among solution branches corresponding to the same non-strict nodal contact modes except for a branch in the opposite direction in the case when the starting point corresponds to a solution with strict nodal contact modes only. 


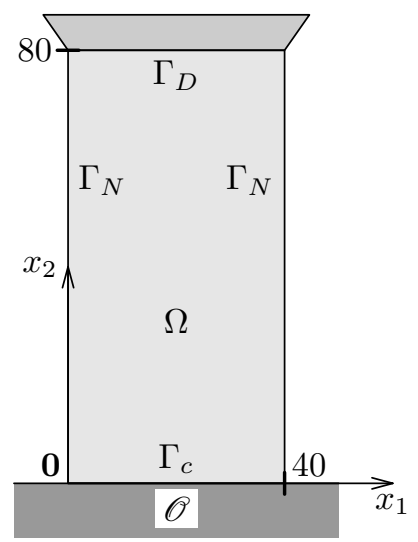

(a)

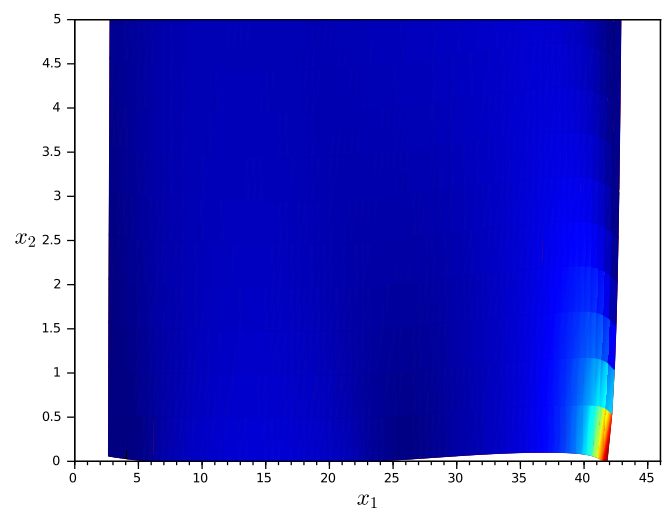

(c)

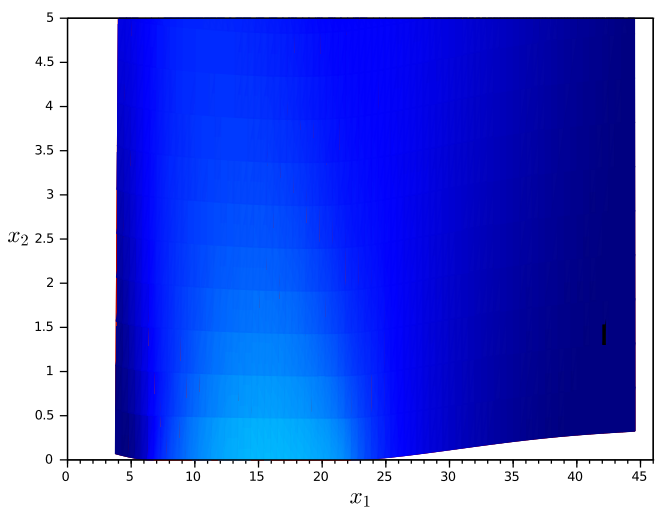

(b)

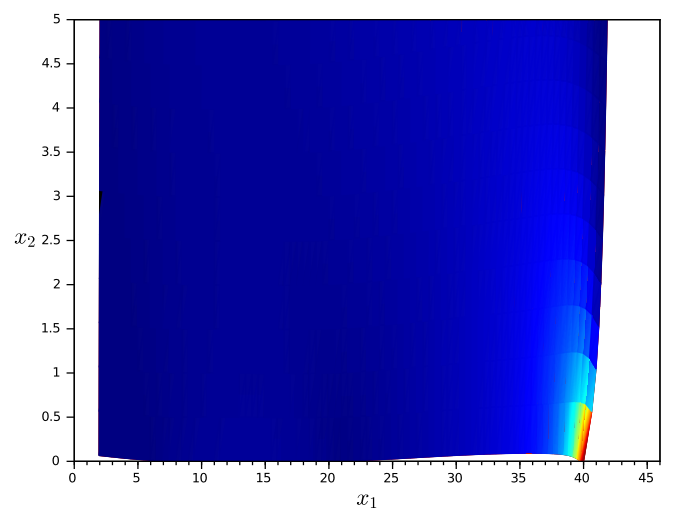

(d)

Figure 13: Rectangular block in contact with a rigid foundation: (a) reference configuration; (b)-(d) three solutions for $\gamma=0.495$ and $\mathscr{F}=1.8$.

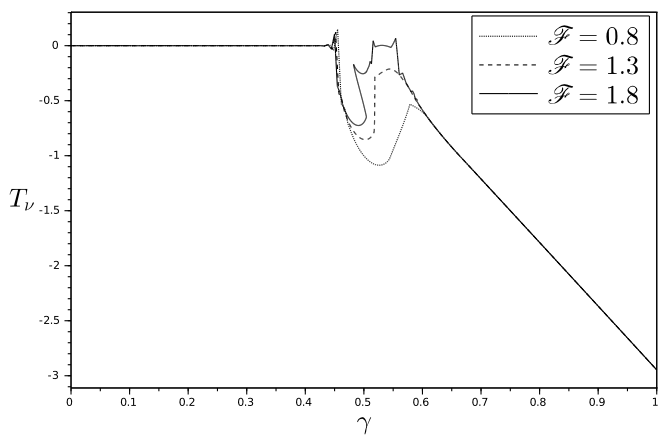

Figure 14: The normal contact stress at the node with the coordinates $(10 \mathrm{~mm}, 0 \mathrm{~mm})$ in the reference configuration. 
An easy-to-implement restarted predictor-corrector continuation method has been proposed for general $P C^{1}$ solution curves. It has been tested on contact problems with large numbers of degrees of freedom (related to two-dimensional problems) and solution curves with non-smooth folds have been recovered. Comparisons of solution curves corresponding to various mesh sizes and various friction coefficients have been done.

It is worth noticing that the particular continuation problem covers also discretised static contact problems with Coulomb friction as its special case. Clearly, multiple solutions of the continuation problem with the same value of the parameter $\gamma$ represent multiple solutions of the static problem for the corresponding data (the external forces or the prescribed displacement on the Dirichlet part of the boundary) in this case. As the structure of solutions of static problems has not been fully understood yet, neither for a model of small-deformation elasticity, the present work may be also a step towards better understanding of these problems.

\section{Acknowledgements}

Principal results of this paper were achieved at a postdoctoral stay of T. Ligurský at INSA-Lyon funded by Manufacture Française des Pneumatiques Michelin. During writing of the paper, T. Ligurský received support from the project "Support for building excellent research teams and inter-sectoral mobility at Palacký University in Olomouc II" (CZ.1.07/2.3.00/30.0041). The authors would also like to thank anonymous referees for careful reading of the manuscript and their numerous comments that have helped to improve the exposition.

\section{References}

[1] P. Alart, Critères d'injectivité et de surjectivité pour certaines applications de $\mathbf{R}^{n}$ dans lui-même ; application à la mécanique du contact. RAIRO Modél. Math. Anal. Numér. 27 (1993), no. 2, 203-222.

[2] P. Alart And A. Curnier, A mixed formulation for frictional contact problems prone to Newton like solution methods. Comput. Methods Appl. Mech. Engrg., 92 (1991), no. 3, 353-375.

[3] J. C. Alexander, T.-Y. Li And J. A. Yorke, Piecewise smooth homotopies. In: Homotopy Methods and Global Convergence, NATO Conf. Ser. II: Systems Sci., Vol. 13, Plenum, New York , 1983, 1-14.

[4] E. L. Allgower and K. Georg, Numerical path following. In: Handbook of Numerical Analysis, Vol. V, North-Holland, Amsterdam, 1997, 3-207. 
[5] P. Beremlijski, J. Haslinger, M. Kočvara and J. V. Outrata, Shape optimization in contact problems with Coulomb friction. SIAM J. Optim. 13 (2002), no. 2, $561-587$.

[6] G. BJöRkman, Path following and critical points for contact problems. Comput. Mech. 10 (1992), no. 3-4, 231-246.

[7] P. G. Ciarlet, Mathematical elasticity. Vol. I: Three-dimensional elasticity. Studies in mathematics and its applications, 20, North-Holland, Amsterdam, 1988.

[8] A. Dhooge, W. Govaerts and Yu. A. Kuznetsov, matCont: a matlaB Package for Numerical Bifurcation Analysis of ODEs. ACM Trans. Math. Software 29 (2003), no. 2, 141-164.

[9] F. FACChinei and J.-S. PANG, Finite-Dimensional Variational Inequalities and Complementarity Problems. Vol. II. Springer Series in Operations Research, Springer, New York, 2003.

[10] R. Goldman, Curvature formulas for implicit curves and surfaces. Comput. Aided Geom. Design 22 (2005), no. 7, 632-658.

[11] J. Haslinger, V. JanovskÝ and R. KuČEra, Path-following the static contact problem with Coulomb friction. In: Proceedings of the International Conference Applications of Mathematics 2013: Institute of Mathematics, Academy of Sciences of the Czech Republic, Prague 2013, 104-116.

[12] J. Haslinger, V. JanOvskÝ And T. LiguRskÝ, Qualitative analysis of solutions to discrete static contact problems with Coulomb friction. Comput. Methods Appl. Mech. Engrg. 205-208 (2012), 149-161.

[13] P. Hild, On finite element uniqueness studies for Coulomb's frictional contact model. Int. J. Appl. Math. Comput. Sci. 12 (2002), no. 1, 41-50.

[14] V. JanovskÝ and T. LigurskÝ, Computing non unique solutions of the Coulomb friction problem. Math. Comput. Simulation 82 (2012), no. 10, 2047-2061.

[15] Y. KanNo AND M. OHSAKI, A non-interior implicit smoothing approach to complementarity problems for frictionless contacts. Comput. Methods Appl. Mech. Engrg. 200 (2011), no. 9-12, 1176-1185.

[16] H. B. Keller, Lectures on Numerical Methods in Bifurcation Problems. Lectures on Mathematics and Physics, 79, Tata Institute of Fundamental Research, Bombay, 1987.

[17] A. KLARBring, Examples of non-uniqueness and non-existence of solutions to quasistatic contact problems with friction. Ingenieur-Archiv 60 (1990), no. 8, 529-541. 
[18] A. Klarbring, Contact, Friction, Discrete Mechanical Structures and Mathematical Programming. In: New Developments in Contact Problems, CISM Courses and Lectures, Vol. 384, Springer, Wien, 1999, 55-100.

[19] A. Klarbring, Stability and Critical Points in Large Displacement Frictionless Contact Problems. In: Friction and Instabilities, CISM Courses and Lectures, 457, Springer, Vienna, 2002, 39-64.

[20] H. B. Khenous, J. Pommier and Y. Renard, Hybrid discretization of the Signorini problem with Coulomb friction. Theoretical aspects and comparison of some numerical solvers. Appl. Numer. Math. 56 (2006), no. 2, 163-192.

[21] T. LiguRSKÝ, Theoretical analysis of discrete contact problems with Coulomb friction. Appl. Math. 57 (2012), no. 3, 263-295.

[22] A. Pinto da Costa And J. A. C. Martins, The evolution and rate problems and the computation of all possible evolutions in quasi-static frictional contact. Comput. Methods Appl. Mech. Engrg. 192 (2003), no. 26-27, 2791-2821.

[23] A. Pinto da Costa And J. A. C. Martins, A numerical study on multiple rate solutions and onset of directional instability in quasi-static frictional contact problems. Comput. Struct. 82 (2004), no. 17-19, 1485-1494.

[24] D. Ralph And S. Scholtes, Sensitivity analysis of composite piecewise smooth equations. Math. Programming 76 (1997), no. 3, Ser. B, 593-612.

[25] Y. Renard and J. Pommier, GetFeM++. An open source generic C++ library for finite element methods (http://home.gna.org/getfem).

[26] S. Scholtes, Introduction to piecewise differentiable equations. Preprint No. 53/1994, Institut für Statistik und Mathematische Wirtschaftstheorie, Universität Karlsruhe, 1994.

[27] M. Schultz And S. Pellegrino, Equilibrium paths of mechanical systems with unilateral constraints. I. Theory. Proc. R. Soc. Lond. Ser. A Math. Phys. Eng. Sci. 456, (2000) 2223-2242.

[28] H. Sellami and S. M. Robinson, Implementation of a continuation method for normal maps. Math. Programming 76 (1997), no. 3, Ser. B, 563-578.

[29] S. M. Sun, M. C. Natori, Numerical solution of large deformation problems involving stability and unilateral constraints. Comput. Struct. 58 (1996), no. 6, 1245-1260. 